\title{
Automorphisms in Loop Quantum Gravity
}

\author{
Benjamin Bahr, MPI für Gravitationsphysik, Albert-Einstein Institut, \\ Am Mühlenberg 1, 14467 Golm, Germany \\ Thomas Thiemann, MPI für Gravitationsphysik, Albert-Einstein Institut, \\ Am Mühlenberg 1, 14467 Golm, Germany; \\ Perimeter Institute for Theoretical Physics, \\ 31 Caroline St. N., Waterloo Ontario N2L 2Y5, Canada
}

October 27, 2018

\begin{abstract}
We investigate a certain distributional extension of the group of spatial diffeomorphisms in Loop Quantum Gravity. This extension, which is given by the automorphisms $\mathcal{A} u t(\mathcal{P})$ of the path groupoid $\mathcal{P}$, was proposed by Velhinho and is inspired by category theory. This group is much larger than the group of piecewise analytic diffeomorphisms. In particular, we will show that graphs with the same combinatorics but different generalized knotting classes can be mapped into each other. We describe the automorphism-invariant Hilbert space and comment on how a combinatorial formulation of LQG might arise.
\end{abstract}

\section{Introduction}

Loop Quantum Gravity is an attempt to quantize the theory of general relativity (see $[1,2,3,4]$ and references therein). This is done by casting classical GR into a hamiltonian formulation, in which it becomes a constrained theory with fields on a Cauchy hypersurface $\Sigma$. These fields are an $\mathfrak{s u}(2)$-connection $A_{a}^{I}(x)$ (the Ashtekar connection) and its canonically conjugate $E_{I}^{a}(x)$, which is the analogue of the electric field in $S U(N)$-Yang-Mills theory. Modulo physical constants, which can be absorbed into the definition of $E_{I}^{a}(x)$, the Poisson structure reads

$$
\left\{A_{a}^{I}(x), E_{J}^{b}(y)\right\}=\delta_{a}^{b} \delta_{J}^{I} \delta(x, y) .
$$

The connection $A$ plays the rôle of the configuration variable, and $E$ is the canonically conjugate momentum. The classical configuration space is the space of smooth connections $\mathcal{A}$.

By going over to the quantum regime, the space $\mathcal{A}$ is extended to $\overline{\mathcal{A}}$, by adding also connections which are "distributional" in the sense that they can have support 
on lower-dimensional subspaces of $\Sigma$. The space $\overline{\mathcal{A}}$ can be endowed with a topology that turns it into a compact Hausdorff space, and a regular, normalized Borel measure $\mu_{A L}$, the Ashtekar-Isham-Lewandowski-measure. The quantum theory is then formulated in the kinematical Hilbert space $\mathcal{H}_{\text {kin }}=L^{2}\left(\overline{\mathcal{A}}, d \mu_{A L}\right)$. The space $\mathcal{A}$ is topologically dense in $\overline{\mathcal{A}}$, but measure-theoretically thin: $\mathcal{A}$ is contained in a set of measure zero.[4]

This is analogous to the case of quantum field theory: Consider the case of the Euclidean free scalar field. Then the path integral formalism proposes to write the partition function $Z([J])$ as an integral over all field configurations

$$
Z[J]=Z[0] \int \mathcal{D} \phi e^{-\int\left[\phi^{*}\left(\square+m^{2}\right) \phi+i \phi J\right]}
$$

In this case, the integration ranges over a set of field configurations $\phi$, in which the smooth fields lie dense. The set of these fields, however, are contained in a set of measure zero.

In the Hamiltonian formulation of GR employed in Loop Quantum Gravity, the dynamics is contained in the constraint functions, which are phase space functions that generate a Hamiltonian flow on the constraint hypersurface. Two of the constraints, the Gauss- and the Diffeomorphism- constraint, encode the invariance of the theory under change of $S U(2)$-gauge and diffeomorphisms on $\Sigma$. Consequently, their Hamiltonian flows generate gauge-transformations and diffeomorphisms on $\Sigma$, respectively. Classically, the corresponding group of smooth gauge transformations $\mathcal{G}$ and diffeomorphisms $\operatorname{Diff}(\Sigma)$ act on $\mathcal{A}$ :

$$
\begin{aligned}
\alpha_{g}: \mathcal{A} \longrightarrow \mathcal{A}, & g \in \mathcal{G} \\
\alpha_{\phi}: \mathcal{A} \longrightarrow \mathcal{A}, & \phi \in \operatorname{Diff}(\Sigma) .
\end{aligned}
$$

The action of the groups $\mathcal{G}$ and $\operatorname{Diff}(\Sigma)$ can be easily extended to the quantum configuration space $\overline{\mathcal{A}}$. But also the groups themselves can be extended to groups of generalized gauge transformations and generalized diffeomorphisms, $\overline{\mathcal{G}}$ and $\overline{\operatorname{Diff}(\Sigma)}$, to give

$$
\begin{aligned}
\alpha_{g}: \overline{\mathcal{A}} \longrightarrow \overline{\mathcal{A}}, & g \in \overline{\mathcal{G}} \\
\alpha_{\phi}: \overline{\mathcal{A}} \longrightarrow \overline{\mathcal{A}}, & \phi \in \overline{\operatorname{Diff}(\Sigma)} .
\end{aligned}
$$

The group of generalized gauge transformation $\overline{\mathcal{G}}$ is taken to be the group of all maps from $\Sigma$ to $S U(2)$, i.e.

$$
\overline{\mathcal{G}}=S U(2)^{\Sigma}
$$

Although classical GR is not invariant under this group, in the quantum theory this arises as a natural candidate for the extension of the smooth gauge transformations.

There are reasons to believe that also the diffeomorphisms have to be extended: First, the Hilbert space $\mathcal{H}_{\text {diff }}$ of states invariant under diffeomorphisms, is not separable and contains many degrees of freedom (the so-called moduli) that are believed 
to be unphysical $[4,5]$. Also, there are other physical reasons to believe that the group of diffeomorphisms is too small [6]. For an extension of the diffeomorphisms $\operatorname{Diff}(\Sigma)$ to $\overline{\operatorname{Diff}(\Sigma)}$, several suggestions have been made.

In [5], it was shown that already a slight extension of the group of diffeomorphisms gives rise to a separable diff-invariant Hilbert space. In [7], Ashtekar and Lewandowski discussed $C^{n}$-diffeomorphisms on $\Sigma$, which are analytic except for lower-dimensional subsets of $\Sigma$. The proof of the uniqueness of the diffeomorphisminvariant state $\omega_{A L}$ on the holonomy-flux-algebra for Loop Quantum Gravity uses these for $n \geq 1$ [8]. In [6], the stratified diffeomorphisms, introduced earlier by Fleischhack have been investigated. In [9] the piecewise analytic diffeomorphisms have been introduced, which are bijections on $\Sigma$ that leave the set of analytical graphs $\Gamma$ invariant. In [10], the graphomorphisms extended this concept to the smooth and other categories.

In $[11,12]$, it was displayed how the basic ingredients of Loop Quantum Gravity can be formulated naturally as concepts of category theory, i.e. as morphisms, functors and natural transformations. In this language, the connections arise as functors from the path groupoid $\mathcal{P}$ of $\Sigma$ to the suspension of the gauge group $\operatorname{Susp}(S U(2))$, and the generalized gauge transformations are in one-to-one correspondence to the natural transformations of these functors. Furthermore, the diffeomorphisms acting on $\Sigma$ can be naturally interpreted as elements in the automorphism group $\mathcal{A} u t(\mathcal{P})$, i.e. as invertible functors from $\mathcal{P}$ to itself. Velhinho pointed out that in the light of category language, $\mathcal{A} u t(\mathcal{P})$ arises as a candidate for an extension of the diffeomorphisms $\operatorname{Diff}(\Sigma)$, and this extension appears to be natural, at least from the mathematical point of view.

In this article, we will investigate the consequences of choosing $\overline{\operatorname{Diff}(\Sigma)}=\mathcal{A} u t(\mathcal{P})$. The automorphisms $\phi \in \mathcal{A} u t(\mathcal{P})$ are invertible functors on the path groupoid $\mathcal{P}$, i.e. they permute points in $\Sigma$, and also the paths between them in a consistent way. We will, however, encounter elements in $\mathcal{A} u t(\mathcal{P})$ that can not be interpreted as bijections of $\Sigma$. By this, the elements in $\mathcal{A} u t(\mathcal{P})$ will also be able to map graphs into each other that have the same combinatorics, but lie in different generalized knotting classes. By this, a combinatorial picture emerges, which is a desirable feature for a quantum theory of gravity $[4,13,14]$.

The emphasis of this article lies on two topics: First, we will prove that the automorphisms $\mathcal{A} u t(\mathcal{P})$ leave the Ashtekar-Isham-Lewandowski measure $\mu_{A L}$ invariant, and hence have a well-defined unitary action on the kinematical Hilbert space $\mathcal{H}_{\text {kin }}=L^{2}\left(\overline{\mathcal{A}}, d \mu_{A L}\right)$. Second, we will have a closer look at the automorphisms and the orbits of its action on $\mathcal{H}_{\text {kin }}$, in order to describe the automorphism-invariant Hilbert space.

We will start in chapter 2 by reviewing the basic concepts of Loop Quantum Gravity, with emphasis on the categorial formulation, and for general gauge group $G$. We introduce the concept of a (primitive) metagraph, which will be useful in the investigation of the automorphisms $\mathcal{A} u t(\mathcal{P})$. We will continue by presenting two kinds of nontrivial automorphisms in section 3, which will both be not induced by a bijection on $\Sigma$, but are most useful in what follows. In particular, with the help of these automorphisms we will prove in chapter 4 that the automorphisms leave the Ashtekar-Isham-Lewandowski measure invariant, but also be able to show that any two graphs (in fact, hyphs) with the same combinatorics can be mapped into 
each other by an automorphism. It is in particular this fact which suggests that by using the automorphisms, a combinatorial picture emerges.

In section 5 we will investigate the orbits of vectors in $\mathcal{H}_{\text {kin }}$ under the action of $\mathcal{A} u t(\mathcal{P})$. For a certain choice of rigging map, we will define the Hilbert space of vectors invariant under the action of $\mathcal{A} u t(\mathcal{P})$. For the case of abelian Loop Quantum Gravity, i.e. $G=U(1)$, an explicit orthonormal basis will be given. for $G=S U(2)$, we will comment on how to obtain such a basis.

In the appendix, we will, after briefly presenting notions from category theory and combinatorial group theory, present a way of how to write the exponentiated fluxes in category language, and present a categorial version of the Weyl- and the holonomy-flux algebra.

\section{Basics}

Loop Quantum Gravity rests on the observation that, instead of knowing a $\mathfrak{g}$ connection $A_{a}$, it is equivalent to know, for every possible path $p$, its parallel transport $A(p)=\mathcal{P} \exp \int_{p} A_{a} d x^{a}$ along $p$, which is an element of the gauge group $G$. These parallel transports, or holonomies, form the configuration space $\mathcal{A}$ of the classical theory, which is extended to $\overline{\mathcal{A}}$, the set of generalized connections, in the quantum theory. The close relation to lattice gauge theory is rooted in this formalism.

\subsection{Curves, ways and paths}

In the following chapter we will review the basic notions to deal with the set of generalized connections, as well as their categorical formulation ${ }^{1}$. For a more detailed mathematical treatment and the omitted proofs, see e.g. $[4,15,16]$. Also, we will restrict ourselves to the category of piecewise analytic paths, which is usually employed in Loop Quantum Gravity. In all what follows, $\Sigma$ will denote an analytic, connected, closed manifold of dimension $n>2$.

Definition 2.1 Let $\Sigma$ be an analytic, connected, closed manifold of dimension $n>$ 2. A (piecewise analytic) curve in $\Sigma$ is a map $c:[0,1] \rightarrow \Sigma$, such that there exist $0=t_{0}<t_{1}<\ldots<t_{n}=1$, so that $c$ restricted to $\left[t_{k}, t_{k+1}\right]$ is an analytic embedding. Denote the set of all curves as $\mathcal{C}$. Write

- $s(c):=c(0)$

- $t(c):=c(1)$

- $r(c):=c([0,1]) \subset \Sigma$.

Some curves can be concatenated, and all of them can be inverted:

Definition 2.2 Let $c_{1}, c_{2} \in \mathcal{C}$ such that $t\left(c_{1}\right)=s\left(c_{2}\right)$. Then

$$
c_{1} \circ c_{2}(t):=\left\{\begin{array}{cc}
c_{1}(2 t) & t \in\left[0, \frac{1}{2}\right] \\
c_{2}(1-2 t) & t \in\left[\frac{1}{2}, 1\right]
\end{array}\right.
$$

\footnotetext{
${ }^{1}$ We follow the notation of [4], where the concatenation of, say, two curves $c_{1}$, and $c_{2}$ is denoted as $c_{1} \circ c_{2}$, rather than $c_{2} \circ c_{1}$, which is usually employed in category theory texts.
} 
defines an element in $\mathcal{C}$, which is called the product of $c_{1}$ and $c_{2}$. Furthermore, for any $c \in \mathcal{C}$ :

$$
c^{-1}(t):=c(1-t)
$$

also defined an element in $c^{-1} \in \mathcal{C}$ which is called the inverse of $c$.

All this endows $\mathcal{C}$ with some structure, but so far the multiplication $\circ$ is not associative.

Definition 2.3 Define an equivalence relation on curves: $c_{1} \sim c_{2}$ if there is a piecewise analytic, monotonically increasing function $\varphi:[0,1] \rightarrow[0,1]$ such that $c_{1}(t)=c_{2}(\varphi(t))$. So two curves $c_{1}, c_{2}$ are equivalent if and only if there is a sequence of curves $d_{1}, \ldots, d_{n} \in \mathcal{C}$ such that $d_{k} \sim d_{k+1}$ in the above sense and $d_{1}=c_{1}, d_{n}=c_{2}$.

The set of all equivalence classes $[c]$ is called the set of ways, and is denoted by $\tilde{\mathcal{C}}$. For $w=[c] \in \tilde{\mathcal{C}}, s(w):=s(c), t(w):=t(c)$ and $r(w):=r(c)$ are well-defined.

The set $\tilde{\mathcal{C}}$ carries the structure of a category in the following way: The objects of $\tilde{\mathcal{C}}$ are points in $\Sigma:|\tilde{\mathcal{C}}|=\Sigma$. For $x, y \in \Sigma$ the set of morphisms $\operatorname{Mor}_{\tilde{\mathcal{C}}}(x, y)$ is given by the set of all ways $w$ starting at $x$ and ending at $y$, i.e. $s(w)=x, t(w)=y$. The concatenation $\left[c_{1}\right] \circ\left[c_{2}\right]:=\left[c_{1} \circ c_{2}\right]$ is well-defined and associative, since ways do not depend on a parametrization. Also $[c]^{-1}:=\left[c^{-1}\right]$ defines an involution on $\tilde{\mathcal{C}}$. For each $x \in \Sigma$ the identity $\mathrm{id}_{x}$ is given by the equivalence class $[c]$ of the constant curve $c(t)=x$.

Definition 2.4 Let $x, y \in \Sigma$. On the elements of $\operatorname{Mor}_{\tilde{\mathcal{C}}}(x, y)$ define an equivalence relation by: Let $w_{1} \approx w_{2}$ if there are ways $v, v_{1}, v_{2}$ such that $w_{1}=v_{1} \circ v_{2}$ and $w_{2}=v_{1} \circ v \circ v^{-1} \circ v_{2}$. This generates an equivalence relation $\approx$ on $\operatorname{Mor}_{\tilde{\mathcal{C}}}(x, y)$. The set of equivalence classes $[[w]]$ are called paths. The set of paths is denoted by $\mathcal{P}$.

The category which is defined by $|\mathcal{P}|:=|\tilde{\mathcal{C}}|$ and $\operatorname{Mor}_{\mathcal{P}}(x, y):=\operatorname{Mor}_{\tilde{\mathcal{C}}}(x, y) / \approx$, as well as $[[w]] \circ[[v]]:=[[w \circ v]]$ is called the path groupoid, and will also be denoted by $\mathcal{P}$.

Usually, $\mathcal{P}$ is defined by defining an equivalence relation $\approx$ on $\mathcal{C}$ by combining the two equivalence relations above, and directly go from the curves $c$ to their equivalence classes $p=[[[c]]]$. However, in this work it will turn out to be more convenient at some points to work with the category $\tilde{\mathcal{C}}$, so we also define it here.

Also note that $s([[w]]):=s(w)$ and $t([[w]]):=t(w)$ can also be defined in $\mathcal{P}$, but $r([[w]])=r(w)$ is not well-defined. One can define

$$
r(p):=\bigcap_{p=[[w]]} r(w)
$$

but this has the property that $r(p \circ q) \neq r(p) \cup r(q)$. But $r(p \circ q)=r(p) \cup r(q)$ is a desirable property if one wants to speak about "points $x \in \Sigma$ lying on $p$ ". It is this particular fact that makes the set of automorphisms of $\mathcal{P}$ much larger than the set of automorphisms of $\tilde{\mathcal{C}}$. 
Definition 2.5 Let $p$ be a path in $\Sigma$. If there is a representative $c \in \mathcal{C}$ with $p=$ $[[[c]]]$, such that from $c(t)=c\left(t^{\prime}\right)$ it follows that either $t=t^{\prime}$ or $t=0, t^{\prime}=1$ or $t=1, t^{\prime}=0$, then $p$ is said to have no self-intersections.

Note that for paths without self-intersections it is easier to talk about points lying on that path. Let $c$ be a representative of such a path, then $r(p):=r(c)$ is well-defined and gives the same result as (2.3). We will use this notion in some constructions later.

Definition 2.6 $A$ path $e \in \mathcal{P}$ is called an edge if there is a representative $c \in C y l$ with $e=[[[c]]]$, such that $c$ is analytic, and $e$ has no self-intersections.

The edges are special elements in $\mathcal{P}$. They are the equivalence classes of analytic curves that do not self-intersect (unless they start and end at the same point). By definition, every path $p$ can be decomposed into finitely many edges, which will be crucial for the rest of the work.

Edges are the key to gain access to the analytic structure of Loop Quantum Gravity.

Definition 2.7 Let $\gamma=\left\{e_{1}, \ldots, e_{E}\right\}$ be a set of edges in $\mathcal{P}$, such that the following holds: For each $e_{k}$ there is a representative $e_{k}=\left[\left[\left[c_{k}\right]\right]\right]$ in $\mathcal{C}$ such that the $c_{k}$ mutually intersect at most in their beginning- or endpoints. Then $\gamma$ is called a graph. Denote the set of all graphs by $\Gamma$.

Lemma 2.1 Given two graphs $\gamma_{1}, \gamma_{2}$, such that each edge in $\gamma_{1}$ is a finite product of edges and their inverses of $\gamma_{2}$. Then one writes $\gamma_{1} \leq \gamma_{2}$. This defines a partial ordering, in particular for any two graphs $\gamma_{1}, \gamma_{2}$ there is a graph $\gamma_{3}$ such that $\gamma_{1} \leq \gamma_{3}$ and $\gamma_{2} \leq \gamma_{3}$.

This Lemma has an important corollary: Given any finite number of paths $\left\{p_{1}, \ldots, p_{n}\right\}$ in $\mathcal{P}$, there is always a graph $\gamma$ such that each $p_{k}$ is a product of edges (and their inverses) in $\gamma$. For this to hold, the piecewise analyticity of the curves in $\mathbb{C}$ is essential. In particular, the same does not hold if one drops the condition of analyticity and works in the smooth category. However, one can work with the so-called webs, or hyphs, which can also be used in this context and which generalize the concept of graphs. In the analytic category, the definition of hyphs is as follows:

Definition 2.8 Let $v=\left(p_{1}, \ldots, p_{n}\right)$ be a finite sequence of paths with the following property:

For each $k \in\{1, \ldots, n\}$ the path $p_{k}$ has a segment which is free with respect to the $p_{l}, l \in\{1, \ldots, k-1\}$. This means that, given a graph $\gamma$ such that each $p_{k}$ is a product of edges in $\gamma$ and their inverses, then in the decomposition $p_{k}=e_{k_{1}} \circ \ldots \circ e_{k_{n_{k}}}$ there is one edge $e_{k_{l}}$ (or its inverse) which appears exactly once, and which does not appear in the decompositions of all $p_{l}, l \in\{1, \ldots, k-1\}$.

Denote the set of all hyphs by $\mathcal{V}$.

The set of hyphs is also partially ordered. Since all graphs are also hyphs, this is trivial in the analytic category, but stays true in other cases, such as the smooth category as well [16]. Note that in the original definition of a hyph, the additional 
condition that each $p_{k}$ has no self-intersections. This is a much stronger condition, but we will need the weaker definition presented here later.

In Loop Quantum Gravity, GR is written in terms of a gauge theory, i.e. the notion of a compact, connected, semisimple Lie Group $G$ is employed. The following definition a priori depends on this gauge group. For LQG, the cases $G=S U(2)$ and $G=U(1)$ are the most important.

Definition 2.9 Let $G$ be a semisimple, connected, compact Lie group. Let furthermore $\mu=\left\{p_{1}, \ldots, p_{M}\right\}$ be a set of paths in $\mathcal{P}$ that are algebraically independent. This means that for each set $\left\{g_{1}, \ldots, g_{M}\right\}, g_{k} \in G$, there is a functor $A: \mathcal{P} \rightarrow \operatorname{Susp}(G)$ such that

$$
A\left(p_{k}\right)=g_{k} .
$$

Then $\mu$ is called a metagraph. Denote the set of all metagraphs by $\mathcal{M}$.

Note that this notion depends on $G$ : Consider for instance two loops $l_{1} \neq l_{2}$ and $p=l_{1} \circ l_{2} \circ l_{1}^{-1} \circ l_{2}^{-1}$. Then $\{p\}$ is a metagraph for $G=S U(2)$, but is not for $G=U(1)$.

For the gauge groups in question, however, one can show that $\Gamma \subset \mathcal{V} \subset \mathcal{M}$. So, each graph is a hyph, and each hyph is a metagraph, but not the other way round ${ }^{2}$. Define a partial ordering on $\mathcal{M}$ by the same rule as for graphs or hyphs: $\mu_{1} \leq \mu_{2}$ iff every path $p \in \mu_{1}$ can be composed by paths in $\mu_{2}$, or their inverses. Since for every finite set of paths $\left\{p_{k}\right\}$ one can find a graph $\gamma$ so that each $p_{k}$ can be composed of edges in $\gamma$ and their inverses, and each graph is again a metagraph, $\leq$ defines a partial ordering on $\mathcal{M}$.

The metagraphs are a useful concept when investigating the automorphisms $\mathcal{A} u t(\mathcal{P})$, since $\mathcal{A} u t(\mathcal{P})$ leaves $\mathcal{M}$ invariant, which can be seen easily. In contrast, we will encounter explicit examples of elements $\phi \in \mathcal{A} u t(\mathcal{P})$ that do not leave $\Gamma$ or $\mathcal{V}$ invariant. So, the mathematical concept of metagraphs will be quite useful in order to investigate the action of $\mathcal{A} u t(\mathcal{P})$ on $\mathcal{P}$.

\subsection{Notions from Loop Quantum Gravity}

We now briefly review how the concept of the path groupoid $\mathcal{P}$ is used in Loop Quantum Gravity, in particular how to define the quantum configuration space $\overline{\mathcal{A}}$. We will do this in terms staying as close as possible to category language. For a brief introduction to category notions, see appendix A.

Definition 2.10 Let $\mathcal{P}$ be the path groupoid of $\Sigma$, and $G$ a compact, connected, semisimple Lie group. Then a functor $A: \mathcal{P} \rightarrow \operatorname{Susp}(G)$ is called a (generalized) connection $^{3}$. This means that $A$ maps paths in $\mathcal{P}$ to elements in $G$ such that

$$
\begin{gathered}
A(p \circ q)=A(p) \cdot A(q) \\
A\left(p^{-1}\right)=A(p)^{-1} .
\end{gathered}
$$

\footnotetext{
${ }^{2}$ We will see, for instance, that for every path $p$ with $s(p) \neq t(p),\{p\}$ is a metagraph.

${ }^{3}$ In the literature, this space is also denoted as $\operatorname{Hom}(\mathcal{P}, G)$, the set of groupoid homomorphisms from $\mathcal{P}$ to $G$.
} 
The set of all connections is denoted by $\overline{\mathcal{A}}$.

It is clear that every smooth $\mathfrak{s u}(2)$-connection $A_{a}^{I}(x)$ gives rise to such a functor, by mapping each path $p \in \Sigma$ to the holonomy of $A_{a}^{I}$ along $p$ :

$$
A(p):=\mathcal{P} \exp \int_{p} A_{a}^{I} \frac{\tau_{I}}{2} d x^{a}
$$

In this sense the set of all smooth connections $\mathcal{A}$ is a subset of $\overline{\mathcal{A}}$.

Under a gauge transformation $g \in \mathcal{G}=C^{\infty}(\Sigma, S U(2))$, the holonomy $A(p)$ along a path $p$ changes as

$$
A(p) \longrightarrow g_{x} A(p) g_{y}^{-1}
$$

if $p$ starts at $x \in \Sigma$ and ends at $y \in \Sigma$, and $g_{x}$ is the value of the function $g$ at $x \in \Sigma$. This motivates the following definition:

Definition 2.11 A natural transformation $g$ on functors $A \in \overline{\mathcal{A}}$ is called a (generalized) gauge transformation. The set of all such gauge transformations is denoted by $\overline{\mathcal{G}}$.

Recall that functors $A_{1}$ and $A_{2}$ can be related by a natural transformation, if there is for every object $x \in|\mathcal{P}|=\Sigma$ a morphism $g_{x}: A_{1}(x) \rightarrow A_{2}(x)$ such that the following diagram commutes for all $p \in \operatorname{Mor}_{\mathcal{P}}(x, y)$ :

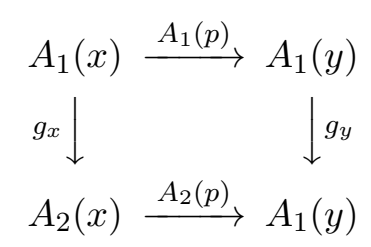

Since $\operatorname{Susp}(G)$ has only one object $*, A(x)=*$ for all $A$ and $x$. This amounts to say that for each $x \in \Sigma$ there is an element $g_{x} \in G$ such that

$$
A_{1}(p)=g_{x} A_{2}(p) g_{y}^{-1} .
$$

This justifies the name gauge transformation, and shows that the set $\overline{\mathcal{G}} \simeq G^{\Sigma}$. Thus, given a functor $A$ and $\left\{g_{x}\right\}_{x \in \Sigma} \in \overline{\mathcal{G}},(2.8)$ can be seen as a definition of the gauge-transformed functor $\alpha_{g} A(p):=g_{s(p)} A(p) g_{t(p)}^{-1}$. So the set $\overline{\mathcal{G}}$ acts on $\overline{\mathcal{A}}$.

This immediately shows that $\overline{\mathcal{G}}=S U(2)^{\Sigma}$, i.e. the set of all maps from $\Sigma$ to $S U(2)$, without any smoothness (or continuity, measurability) condition. It is clear that this is a tremendous extension to a symmetry group which is not a symmetry of classical GR anymore. This shows that the quantum theory is in fact invariant under larger groups, having to do with the fact that space-time becomes discrete in some sense: Gauging can happen at each point in space completely independent of each other. The same can be done for the diffeomorphisms: Every, say analytical, diffeomorphism $\phi$ acts in the path groupoid $\mathcal{P}$ in the following way: points are mapped to points, and paths to paths:

$$
\begin{aligned}
& x \longmapsto \phi(x) \\
& p \longmapsto \phi(p)
\end{aligned}
$$


with $\phi([[[c]]]):=[[[\phi \circ c]]]$ for representative curves $c \in \mathcal{C}$. If $p$ starts at $x$ and ends at $y$, then $\phi(p)$ starts at $\phi(x)$ and ends at $\phi(y)$. This means that $\phi$ induces a functor on the path groupoid $\mathcal{P}$, which is invertible since $\phi$ is as a map.

Definition 2.12 Let $\phi: \mathcal{P} \rightarrow \mathcal{P}$ be an invertible functor. Then $\phi$ is called an automorphism of $\mathcal{P}$. Denote the set of all automorphisms by $\mathcal{A} u t(\mathcal{P})$.

Note that $\mathcal{A} u t(\mathcal{P})$ also acts on $\overline{\mathcal{A}}$, via $\alpha_{\phi} A(p):=A(\phi(p))$. By this, $\mathcal{A} u t(\mathcal{P})$ appears as an extension of $\operatorname{Diff}(\Sigma)$.

It is this set of automorphisms $\mathcal{A} u t(\mathcal{P})$ that we will focus our attention on for the rest of the article. The automorphisms extend the analytic diffeomorphisms, and is the largest possible extension [12]. We will comment on the actual size of $\mathcal{A} u t(\mathcal{P})$ in contrast to $\operatorname{Diff}(\Sigma)$ later in this article. It should be noted that each invertible functor $\zeta: \tilde{\mathcal{C}} \rightarrow \tilde{\mathcal{C}}$, i.e. $\zeta \in \mathcal{A} u t(\tilde{\mathcal{C}})$ induces also an element $\phi_{\zeta} \in \mathcal{A} u t(\mathcal{P})$. In particular, the set $\left\{\phi_{\zeta} \mid \zeta \in \mathcal{A} u t(\tilde{\mathcal{C}})\right\}$ forms a subgroup of $\mathcal{A} u t(\mathcal{P})$, which has been investigated in the literature $[10,13]$, called "piecewise analytic diffeomorphisms", or "graphomorphisms". However this is a proper subgroup: As we will see later, there are many automorphisms in $\mathcal{A} u t(\mathcal{P})$ which are no graphomorphisms.

The reason for this can be seen as follows: The automorphisms permute points of $\Sigma$, and also permutes the paths between points, in a consistent way. Consistent means that if $p \in \operatorname{Mor}_{\mathcal{P}}(x, y)$, then $\phi(p) \in \operatorname{Mor}_{\mathcal{P}}(\phi(x), \phi(y))$. However, a path itself consists (if it is without self-intersection) of many points. So one could feel that for $z$ lying on $p$ (more precisely: $z \in r(p)$ ), then also $\phi(z)$ should lie on $\phi(p)$. Even more, since $p$ can be decomposed into $p_{1} \circ p_{2}$, where $p_{1}$ is the part of $p$ which goes from $x$ to $z$, and $p_{2}$ is the remainder, which goes from $z$ to $y$. Since $\phi(p)=\phi\left(p_{1} \circ p_{2}\right)=\phi\left(p_{1}\right) \circ \phi\left(p_{2}\right)$, one might think that, since $\phi\left(p_{1}\right)$ ends at $\phi(z)$, and $\phi\left(p_{2}\right)$ starts at $z, \phi(p)$ should pass through $\phi(z)$. But this is not the case, as the following picture shows:

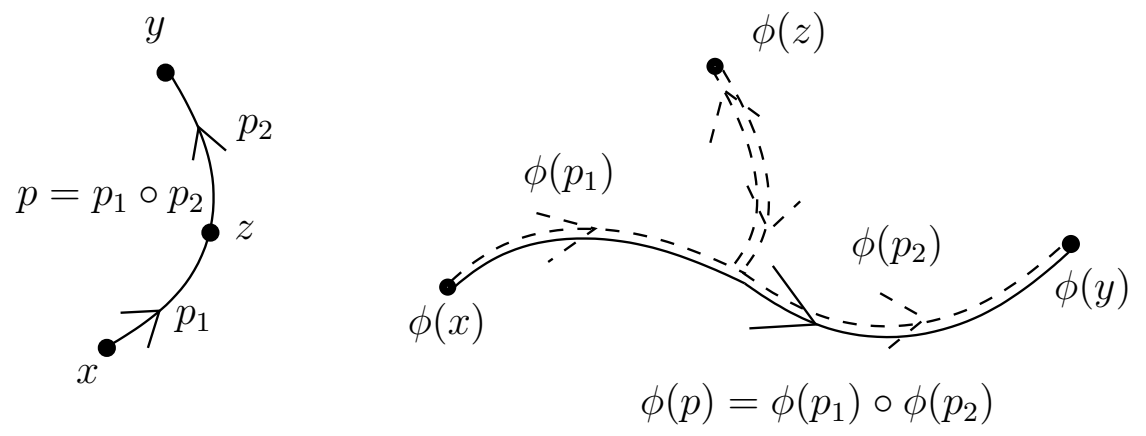

Figure 2.1: Here $z$ lies on $r(p)$, but $\phi(z)$ does not lie on $r(\phi(p))$.

Let $p$ be a path without self-intersections, which is composed of $p=p_{1} \circ p_{2}$, i.e. $p$ passes through $z:=t\left(p_{1}\right)$. The images of $p_{1}$ and $p_{2}$ under $\phi$ are given by the dashed lines, the solid line is $\phi(p) . \phi\left(p_{1}\right)$ ends at $\phi(z)$ and $\phi\left(p_{2}\right)$ starts at it, but since $\phi\left(p_{1}\right) \circ \phi\left(p_{2}\right)$ contains a retracing, $\phi(p)=\phi\left(p_{1}\right) \circ \phi\left(p_{2}\right)$ does not pass through $z$. We see that the fact that retracings cancel out in $\mathcal{P}$, the ill-definedness of $r(p)$, and the existence of automorphisms which are not induced by maps from $\Sigma$ to $\Sigma$, are deeply interrelated to each other. 


\subsection{Automorphisms and connections}

In this section we will investigate the metagraphs further, and their relation to the automorphisms. We will extend the LQG-notions for graphs [15] to metagraphs, and in particular show that the topology on $\overline{\mathcal{A}}$ can also be defined in terms of metagraphs. This will allow to prove i.e. continuity of the action of automorphisms $\mathcal{A} u t(\mathcal{P})$ on the set of connections $\overline{\mathcal{A}}$. So, fix a compact, connected and semisimple Lie group $G$ for the rest of this section. We will refer to $G$ as the gauge group.

The partial ordering defined on the set of metagraphs $\mathcal{M}$ has a category theory background, which we will use in the following.

Definition 2.13 Let $\mathcal{P}$ be the path groupoid of $\Sigma$, and $\mu \in \mathcal{M}$ a metagraph. Then define $\mathcal{P}_{\mu}$ to be the subgroupoid of $\mathcal{P}$ which is generated by the elements in $\mu$.

This groupoid contains the elements in $\mu$, their inverses, the identities $\operatorname{id}_{s\left(p_{k}\right)}, \operatorname{id}_{t\left(p_{k}\right)}$, and all products that can be formed of them. Thus all $\mathcal{P}_{\mu}$ are finitely generated subgroupoids of $\mathcal{P}^{4}$. Note that by this definition, also $\mathcal{P}_{\gamma}$ for $\gamma \in \Gamma$ and $\mathcal{P}_{v}$ for $v \in \mathcal{V}$ are declared.

We immediately conclude:

Corollary 2.1 For two metagraphs $\mu_{1}, \mu_{2} \in \mathcal{M}$, we have that $\mu_{1} \leq \mu_{2}$ if and only if $\mathcal{P}_{\mu_{1}}$ is a subgroupoid of $\mathcal{P}_{\mu_{2}}$.

The same holds for graphs and hyphs. In fact, a topology on the set $\overline{\mathcal{A}}$ of generalized connections $A$, or equivalently functors $A: \mathcal{P} \rightarrow \operatorname{Susp}(G)$ is defined by convergence on the finitely generated subgroupoids $\mathcal{P}_{\gamma}$ for al $\gamma \in \Gamma$. In [16] it was shown that the same topology is defined if one replaces $\Gamma$ by $\mathcal{V}$. In fact, defining the topology on $\overline{\mathcal{A}}$ by using the metagraphs leads to the same result, as we will briefly indicate in the following.

Definition 2.14 Let $\mathcal{P}$ be the path groupoid, and $\overline{\mathcal{A}}$ the set of all morphisms $A$ : $\mathcal{P} \rightarrow \operatorname{Susp}(G)$. For a metagraph $\mu$ denote the set of all morphisms from $\mathcal{P}_{\mu}$ to $\operatorname{Susp}(G)$ by $\overline{\mathcal{A}}_{\mu}$. Define the projection

$$
\begin{aligned}
& \pi_{\mu}: \overline{\mathcal{A}} \longmapsto \overline{\mathcal{A}}_{\mu} \\
& \left(\pi_{\mu} A\right)\left(p_{k}\right):=A\left(p_{k}\right)
\end{aligned}
$$

For $\mu_{1} \leq \mu_{2}$ define the projections $\pi_{\mu_{1} \mu_{2}}: \overline{\mathcal{A}}_{\mu_{2}} \rightarrow \overline{\mathcal{A}}_{\mu_{1}}$ via

$$
\left(\pi_{\mu_{1} \mu_{2}} A\right)(p):=A\left(q_{1}\right) \cdot \ldots \cdot A\left(q_{n}\right)
$$

if $p \in \mu_{1}$ can be written by $p=q_{1} \circ \ldots \circ q_{n}$, the $q_{k}$ being elements in $\mu_{2}$ or their inverses.

It is easy to show that $\pi_{\mu_{1}, \mu_{2}} \circ \pi_{\mu_{2}}=\pi_{\mu_{1}}$ for $\mu_{1} \leq \mu_{2}$. By the definition of metagraphs, each $\overline{\mathcal{A}}_{\mu}, \mu=\left\{p_{1}, \ldots, p_{M}\right\}$ comes with a natural bijection $A \longmapsto$ $\left(A\left(p_{1}\right), \ldots, A\left(p_{M}\right)\right)$. Pulling the topology of $G^{M}$ back to $\overline{\mathcal{A}}_{\mu}$ makes all the $\overline{\mathcal{A}}_{\mu}$ into compact Hausdorff spaces.

\footnotetext{
${ }^{4}$ But not the other way round, as the example for $G=U(1)$ above suggests.
} 
Definition 2.15 Define the topology on $\overline{\mathcal{A}}$ to be the weakest topology such that all the projections $\pi_{\mu}$ are continuous.

One can show that with this topology $\overline{\mathcal{A}}$ becomes a compact Hausdorff space. The proof goes entirely along the same lines as in the case for graphs or hyphs $[4,10]$, and rests crucially on the compactness of $G$. We will not repeat the proof here.

In fact, this topology coincides with the topology which is defined by the condition that all $\pi_{\gamma}$ for $\gamma \in \Gamma$ are continuous.

Lemma 2.2 Let $\mathcal{T}_{1}$ be the weakest topology such that for all $\mu \in \mathcal{M}$ the map $\pi_{\mu}$ is continuous. Let $\mathcal{T}_{2}$ be the weakest topology such that for all graphs $\gamma \in \Gamma$ the map $\pi_{\gamma}$ is continuous. Then the identity map between the two topological space

$$
\text { id }:\left(\overline{\mathcal{A}}, \mathcal{T}_{1}\right) \longrightarrow\left(\overline{\mathcal{A}}, \mathcal{T}_{2}\right)
$$

is a homeomorphism.

Proof: Since $\pi_{\gamma}^{-1}(U)$ are a basis for $\mathcal{T}_{2}$, it is sufficient to show that $\mathrm{id}^{-1}\left(\pi_{\gamma}^{-1}(U)\right)$ are open in $\mathcal{T}_{1}$. But this is clear, since every graph is also a metagraph. Thus, id is a continuous bijection between compact Hausdorff spaces, hence also a homeomorphism.

Thus, defining the topology on $\overline{\mathcal{A}}$ by means of metagraphs is completely analogous to defining it by graphs. However, since the invertible functors $\phi \in \mathcal{A} u t(\mathcal{P})$ leave the set of metagraphs invariant, unlike the set of graphs, in this formulation it is much easier to prove that the automorphisms act continuously on $\overline{\mathcal{A}}$.

Lemma 2.3 Let $\phi: \mathcal{P} \rightarrow \mathcal{P}$ be an invertible functor, i.e. $\phi \in \mathcal{A} u t(\mathcal{P})$. Then the map

$$
\begin{aligned}
& \alpha_{\phi}: \overline{\mathcal{A}} \longrightarrow \overline{\mathcal{A}} \\
& \left(\alpha_{\phi} A\right)(p):=A(\phi(p))
\end{aligned}
$$

is a homeomorphism.

Proof: Let $\mu=\left\{p_{1}, \ldots, p_{M}\right\}$ be a metagraph. In the following, we deliberately use the homeomorphism $\overline{\mathcal{A}}_{\mu} \simeq G^{M}$. For $A \in \overline{\mathcal{A}}$ we have

$$
\begin{aligned}
\pi_{\mu}(A) & =\left(A\left(p_{1}\right), \ldots, A\left(p_{M}\right)\right) \\
& =\left(\alpha_{\phi^{-1}} A\left(\phi\left(p_{1}\right)\right), \ldots, \alpha_{\phi^{-1}} A\left(\phi\left(p_{M}\right)\right)\right) \\
& =\pi_{\phi(\mu)}\left(\alpha_{\phi^{-1}} A\right),
\end{aligned}
$$

i.e. we get

$$
\pi_{\mu} \circ \alpha_{\phi}=\pi_{\phi(\mu)}
$$

From this it follows that, for each open $U \in \overline{\mathcal{A}}_{\mu} \simeq \overline{\mathcal{A}}_{\phi(\mu)}$, one has

$$
\left(\pi_{\phi(\mu)}\right)^{-1}(U)=\left(\alpha_{\phi}\right)^{-1}\left(\left(\pi_{\mu}\right)^{-1}(U)\right)
$$


But since the $\left(\pi_{\mu}\right)^{-1}(U)$ form a basis of the topology on $\overline{\mathcal{A}}$, preimages of open sets under $\alpha_{\phi}$ are open, hence $\alpha_{\phi}$ is continuous. Since this is true for all automorphisms $\phi \in \mathcal{A} u t(\mathcal{P})$ and each $\phi$ is invertible, all automorphisms $\alpha_{\phi}: \overline{\mathcal{A}} \rightarrow \overline{\mathcal{A}}$ are homeomorphisms. This concludes the proof.

There is also a normalized regular Borel measure $\mu_{A L}$ on $\overline{\mathcal{A}}$, which is called the Asthekar-Isham-Lewandowski measure. It is uniquely determined by the condition that its push-forward $\left(\pi_{\gamma}\right)_{*} \mu_{A L}=\mu_{H}$ is the normalized Haar measure on $\overline{\mathcal{A}}_{\gamma} \simeq G^{|\gamma|}$. One could think that the metagraphs $\mu \in \mathcal{M}$ could now used in a similar way to define a measure on $\overline{\mathcal{A}}$, which then would automatically be $\mu_{A L}$. However, the concept of metagraphs is slightly too broad for this: There is in general no measure $\nu$ on $\overline{\mathcal{A}}$ such that $\left(\pi_{\mu}\right)_{*} \nu=\mu_{H}$ on $\overline{\mathcal{A}}_{\mu}$. This can be seen as follows: Choose $G=S U(2)$ and $l \in \mathcal{P}$ be a nontrivial loop, which is also an edge, i.e. $l \in \operatorname{Mor}_{\mathcal{P}}(x, x)$ for some $x \in \Sigma$. Then $\mu_{1}:=\{l\}$ as well as $\mu_{2}:=\left\{l^{2}\right\}$ are metagraphs, since one can take square roots in $S U(2)$. Now $\left(\pi_{\mu_{1}}\right)_{*} \mu_{A L}=\mu_{H}$ on $S U(2)$, but $\left(\pi_{\mu_{2}}\right)_{*} \mu_{A L}=\mu_{H}$ would imply

$$
\int_{S U(2)} d \mu_{H}(h) F(h)=\int_{S U(2)} d \mu_{H}(h) F\left(h^{2}\right)
$$

for all continuous functions $F$ on $S U(2)$. But this is wrong! So, in order to define $\mu_{A L}$ on $\overline{\mathcal{A}}$ without referring to graphs of hyphs, we need the following notion:

Definition 2.16 Let $\mu \in \mathcal{M}$ be a metagraph such that the push-forward of the Ashtekar-Isham-Lewandowksi measure $\mu_{A L}$ by $\pi_{\mu}$ is

$$
\left(\pi_{\mu}\right)_{*} \mu_{A L}=\mu_{H}
$$

the normalized Haar measure $\mu_{H}$ on $\overline{\mathcal{A}}_{\mu} \simeq G^{M}$. Then $\mu$ is called a primitive metagraph.

This notion is the first which is not defined entirely in terms of category theory, but makes use of the analytic structure on $\Sigma$, through the use of the Ashtekar-IshamLewandowski measure, which is defined in terms of graphs (or hyphs). Thus, it is not at all clear whether the automorphisms $\mathcal{A} u t(\mathcal{P})$ preserve the set of primitive metagraphs. Note that this is equivalent to $\left(\alpha_{\phi}\right)^{*} \mu_{A L}=\mu_{A L}$, i.e. the question whether the automorphisms leave $\mu_{A L}$ invariant, or the question whether the induced operators on $\mathcal{H}_{\text {kin }}=L^{2}\left(\overline{\mathcal{A}}, d \mu_{A L}\right)$, given by $(\hat{U}(\phi) \psi)(A):=\psi\left(\alpha_{\phi} A\right)$ are all unitary.

We will provide a proof for this assertion in section 4 . For this proof some explicit examples of automorphisms will play a crucial rôle, which will be presented in the following section.

\section{Examples for automorphisms}

It is obvious that every analytic diffeomorphism $\phi: \Sigma \rightarrow \Sigma$ induces an automorphism $\phi \in \mathcal{A} u t(\mathcal{P})$. In this sense, $\operatorname{Diff}(\Sigma)$ is a subgroup of $\mathcal{A} u t(\mathcal{P})$. But also all 
invertible functors on $\tilde{\mathcal{C}}$, which are in one-to-one correspondence with maps from $\Sigma$ to $\Sigma$ mapping hyphs to hyphs, descend to automorphisms on $\mathcal{P}$ by $\phi[[w]]:=[[\phi(w)]]$.

But, there are many automorphisms $\phi \in \mathcal{A} u t(\mathcal{P})$ that are not induced by a map $\Sigma \rightarrow \Sigma$. The reason for this is deeply connected to the groupoid structure of $\mathcal{P}$. By declaring retracings to be equivalent to the identity $\left[\left[\delta \circ \delta^{-1}\right]\right]=\left[\left[\operatorname{id}_{s(\delta)}\right]\right]$, one disconnects the paths from the points: If $p$ is a path that passes through a point $x$, i.e. $x \in r(p)$, the path $p \circ q$ does not necessarily also have this property. Thus, there is no good notion for a point $x$ lying on a path $p$. This is the reason why there are automorphisms $\phi \in \mathcal{A} u t(\mathcal{P})$ that have actions on points (objects) and paths (morphisms) which are not compatible with each other. In the following, we will give two extreme examples for this:

The first example are the natural transformations of the identity, which arbitrarily permute the points in $\Sigma$, while leaving the paths essentially invariant. These will be crucial in the proof that the automorphisms act unitarily on the kinematical Hilbert space $\mathcal{H}_{\text {kin }}$.

The second example for non-trivial automorphisms will be the edge-interchangers, which interchange two edges with identical beginning- and endpoints, but leave all objects (points $x \in \Sigma$ ) invariant, as well as all paths intersecting the two given edges at most in finitely many points. These will be most helpful in determining the size of the orbits of vectors in $\mathcal{H}_{\text {kin }}$ under the action of $\mathcal{A} u t(\mathcal{P})$, in order to compute the automorphism-invariant Hilbert space $\mathcal{H}_{\text {Aut }}$.

\subsection{Natural transformations of the identity}

Recall from category theory that, given two functors $F, G: \mathcal{C} \rightarrow \mathcal{D}$, the two are called to be natural transformations from each other, if for each object $X$ in $\mathcal{C}$ there is a morphism $g_{X}: F(X) \rightarrow G(X)$ such that the following diagram commutes:

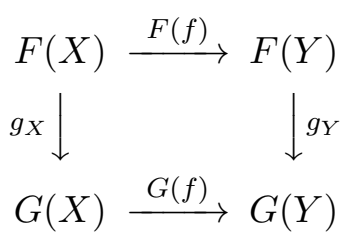

In the context of functors $A: \mathcal{P} \rightarrow \operatorname{Susp}(S U(2))$, two such functors (generalized connections) $A_{1}, A_{2}$ are natural transformations of each other if and only if the one is a gauge transformed of the other. But also automorphisms can be natural transformations of each other. In particular, two automorphisms $\phi_{1}, \phi_{2} \in \mathcal{A} u t(\mathcal{P})$ are natural transformations of each other, if and only if there is a bijection $b: \Sigma \rightarrow \Sigma$ and for each $x \in \Sigma$ a path $p_{x} \in \operatorname{Mor}(x, b(x))$, such that for every path $p \in \operatorname{Mor}(x, y)$ :

$$
\phi_{2}(p)=p_{\phi_{1}(x)}^{-1} \circ \phi_{1}(p) \circ p_{\phi_{1}(y)}
$$

Note that this requires $b(x)=\phi_{2} \circ \phi_{1}^{-1}(x)$. Given $\phi_{1}, b$ and $\left\{p_{x}\right\}_{x \in \Sigma}$, this can also be seen as a definition of the transformed functor $\phi_{2}$. One special case occurs for $\phi_{1}=$ id being the identity functor.

Definition 3.1 Let $b: \Sigma \rightarrow \Sigma$ be a bijection and, for every $x \in \Sigma$ a path $p_{x} \in$ 
$\operatorname{Mor}(x, b(x))$ be given. The functor $\phi_{b, p}$ defined by

$$
\begin{aligned}
& \phi_{b, p}(x):=b(x) \\
& \phi_{b, p}(p):=p_{s(p)}^{-1} \circ p \circ p_{t(p)}
\end{aligned}
$$

is called a natural transformation of the identity.

Given two bijections $b_{1}, b_{2}$ and $p_{x}^{1} \in \operatorname{Mor}\left(x, b_{1}(x)\right)$ and $p_{x}^{2} \in \operatorname{Mor}\left(x, b_{2}(x)\right)$, then one has

$$
\phi_{b_{1}, p^{2}} \circ \phi_{b_{1}, p^{1}}=\phi_{b, p}
$$

with $b:=b_{2} \circ b_{1}$ and $p_{x} \in \operatorname{Mor}\left(x, b_{2}\left(b_{1}(x)\right)\right)$ given by $p_{x}:=p_{x}^{1} \circ p_{b_{1}(x)}^{2}$. In particular, by choosing $b_{2}=b_{1}^{-1}$ and $p_{x}^{2}=\left(p_{b_{1}(x)}^{1}\right)^{-1}$, one sees that every such functor is invertible, hence an automorphism.

Corollary 3.1 The natural transformations of the identity form a subgroup $\mathcal{N}$ of $\mathcal{A} u t(\mathcal{P})$.

The natural transformations of the identity will be of particular importance later on. In particular the group structure will play a prominent role in the proof that all automorphisms act unitarily on $\mathcal{H}_{\text {kin }}$.

\subsection{Edge-interchanger}

The following example of an automorphism will prove to be most important in order to compute the automorphism-invariant Hilbert space $\mathcal{H}_{\text {Aut }}$. It will be an example of a functor which acts trivially on $\Sigma$, but modifies the morphisms. In particular, it will interchange two edges (or paths without self.intersections)) $e_{1}, e_{2}$ with the same starting- and ending point. On the other hand, it will leave every edge which intersects $e_{1}, e_{2}$ in at most finitely many points invariant. It will therefore be termed "edge-interchanger".

Let $e_{1}, e_{2}$ be two paths in $\operatorname{Mor}(x, y)$ for $x \neq y$ without self-intersection, and such that they do not mutually intersect, apart from their beginning- and endpoints. First, choose representative curves $c_{1}, c_{2} \in \mathcal{C}$, i.e. $\left[\left[\left[c_{1}\right]\right]\right]=e_{1},\left[\left[\left[c_{2}\right]\right]\right]=e_{2}$, which contain no retracings, i.e. the maps $c_{1}, c_{2}:[0,1] \rightarrow \Sigma$ are injective. Furthermore, choose for any $t \in(0,1)$ a path $p_{t} \in \operatorname{Mor}_{\mathcal{P}}\left(c_{1}(t), c_{2}(t)\right)$, such that all paths $p_{t}$ have a representative that intersects $r\left(c_{1}\right), r\left(c_{2}\right)$ only at the respective beginningand endpoints. Define $p_{0}=\mathrm{id}_{s\left(e_{1}\right)}$ and $p_{1}=\mathrm{id}_{t\left(e_{1}\right)}$ to be the constant paths. For $t_{1}, t_{2} \in[0,1]$ denote by $c_{1}^{t_{1}, t_{2}}$ the curve

$$
c_{1}^{t_{1}, t_{2}}:[0,1] \ni t \longmapsto c_{1}\left(t_{1}+t\left(t_{2}-t_{1}\right)\right),
$$

and a similar definition of $c_{2}^{t_{1}, t_{2}}$. Note that this definition also makes sense for $t_{1}>t_{2}$, in particular $c_{1}^{t_{1}, t_{2}}=\left(c_{1}^{t_{2}, t_{1}}\right)^{-1}$.

With this data, we now build a functor $\tilde{\phi}: \tilde{\mathcal{C}} \rightarrow \mathcal{P}$ as follows: 
Lemma 3.1 Let $w \in \operatorname{Mor}_{\tilde{\mathcal{C}}}(x, y)$ be a way in $\Sigma$. Then divide $w$ according to the edges $e_{1}, e_{2}$ :

$$
w=w_{1} \circ w_{2} \circ \ldots \circ w_{n} .
$$

where the $w_{k}$ falls into either of the following categories:

- $r\left(w_{k}\right) \cap r\left(e_{1,2}\right)=r\left(w_{k}\right) \Leftrightarrow w_{k}=\left[c_{1}^{t_{1}, t_{2}}\right]$ or $\left[c_{2}^{t_{1}, t_{2}}\right]$

- $r\left(w_{k}\right) \cap r\left(e_{1,2}\right) \subset\left\{s\left(w_{k}\right), t\left(w_{k}\right)\right\}$.

Then the following assignment

$$
\tilde{\phi}(w):=\tilde{\phi}\left(w_{1}\right) \circ \ldots \circ \tilde{\phi}\left(w_{n}\right) \in \operatorname{Mor}_{\mathcal{P}}(x, y)
$$

with

$$
\begin{aligned}
w_{k}=\left[c_{1}^{t_{1}, t_{2}}\right] & \Rightarrow \tilde{\phi}\left(w_{k}\right):=p_{t_{1}} \circ\left[\left[\left[c_{2}^{t_{1}, t_{2}}\right]\right]\right] p_{t_{2}}^{-1} \\
w_{k}=\left[c_{2}^{t_{1}, t_{2}}\right] & \Rightarrow \tilde{\phi}\left(w_{k}\right):=p_{t_{1}}^{-1} \circ\left[\left[\left[c_{1}^{t_{1}, t_{2}}\right]\right]\right] \circ p_{t_{2}} \\
r\left(w_{k}\right) \cap r\left(e_{1,2}\right) \subset\left\{s\left(w_{k}\right), t\left(w_{k}\right)\right\} & \Rightarrow \tilde{\phi}\left(w_{k}\right):=\left[\left[w_{k}\right]\right] .
\end{aligned}
$$

defines a functor $\tilde{\phi}: \tilde{\mathcal{C}} \rightarrow \mathcal{P}$.

Proof: What has to be shown first is that the above assignment is well-defined, i.e. does not depend on the manner the way $w \in \operatorname{Mor}_{\tilde{\mathcal{C}}}(x, y)$ is decomposed w.r.t. the edges $e_{1}, e_{2}$. First we note that, given two decompositions $w_{1} \circ \ldots \circ w_{n}$ and $w_{1}^{\prime} \circ \ldots \circ w_{m}^{\prime}$ of $w$, there is a decomposition $w_{1}^{\prime \prime} \circ \ldots \circ w_{N}^{\prime \prime}$ of $w$ such that each $w_{k}, w_{l}^{\prime}$ is a product of the $w_{r}^{\prime \prime}$. Thus, if we can show that $\tilde{\phi}(w)$ defined by one decomposition of $w$ does not change if we decompose the decomposition further, we are done.

So let $w=w_{1} \circ \ldots \circ w_{n}$ and $w=w_{1}^{\prime} \circ \ldots \circ w_{M}^{\prime}$ be two decompositions of $w$ w.r.t. the edges $e_{1}$ and $e_{2}$, such that each $w_{k}$ is a product of the $w_{l}^{\prime}$. We need to show that

$$
\tilde{\phi}\left(w_{1}\right) \circ \ldots \circ \tilde{\phi}\left(w_{n}\right)=\tilde{\phi}\left(w_{1}^{\prime}\right) \circ \ldots \circ \tilde{\phi}\left(w_{m}^{\prime}\right)
$$

where the $\tilde{\phi}\left(w_{k}\right), \tilde{\phi}\left(w_{l}^{\prime}\right)$ are defined according to (3.8). Let now $w_{k}=\left[c_{1}^{t_{1}, t_{2}}\right]$, and $w_{k}=w_{l}^{\prime} \circ \ldots \circ w_{l+l^{\prime}}^{\prime}$. Then there are points $t_{1}=t_{l}<t_{l+1}<\ldots<t_{l+l^{\prime}+1}=t_{2}$ such that $w_{l+j}^{\prime}=\left[c_{1}^{t_{l+j}, t_{l+j+1}}\right] .5$ Then we have

$$
\begin{aligned}
\tilde{\phi}\left(w_{l}^{\prime}\right) \circ \ldots \circ \tilde{\phi}\left(w_{l+l^{\prime}}^{\prime}\right)= & p_{t_{l}} \circ\left[\left[\left[c_{2}^{t_{l}, t_{l+1}}\right]\right]\right] \circ p_{t_{l}+1}^{-1} \circ p_{t_{l}+1} \circ\left[\left[\left[c_{2}^{t_{l+1}, t_{l+2}}\right]\right]\right] \circ p_{t_{l+2}}^{-1} \circ \ldots \\
& \ldots \circ p_{t_{l+l^{\prime}}}^{-1} \circ p_{t_{l+l^{\prime}}} \circ\left[\left[\left[c_{2}^{t_{l+1}, t_{l+2}}\right]\right]\right] \circ p_{t_{l+l^{\prime}+1}}^{-1} \\
= & p_{t_{l}} \circ\left[\left[\left[c_{2}^{t_{l}, t_{l+1}}\right]\right]\right] \circ \ldots \circ\left[\left[\left[c_{2}^{t_{l+1}, t_{l+2}}\right]\right]\right] \circ p_{t_{l+l^{\prime}+1}}^{-1} \\
= & p_{t_{1}} \circ\left[\left[\left[c_{2}^{t_{l}, t_{l+1}} \circ \ldots \circ c_{2}^{t_{l+1}, t_{l+2}}\right]\right]\right] \circ p_{t_{2}}^{-1} \\
= & p_{t_{1}} \circ\left[\left[\left[c_{2}^{t_{1}, t_{2}}\right]\right]\right] \circ p_{t_{2}}^{-1} \\
= & \tilde{\phi}\left(w_{k}\right) .
\end{aligned}
$$




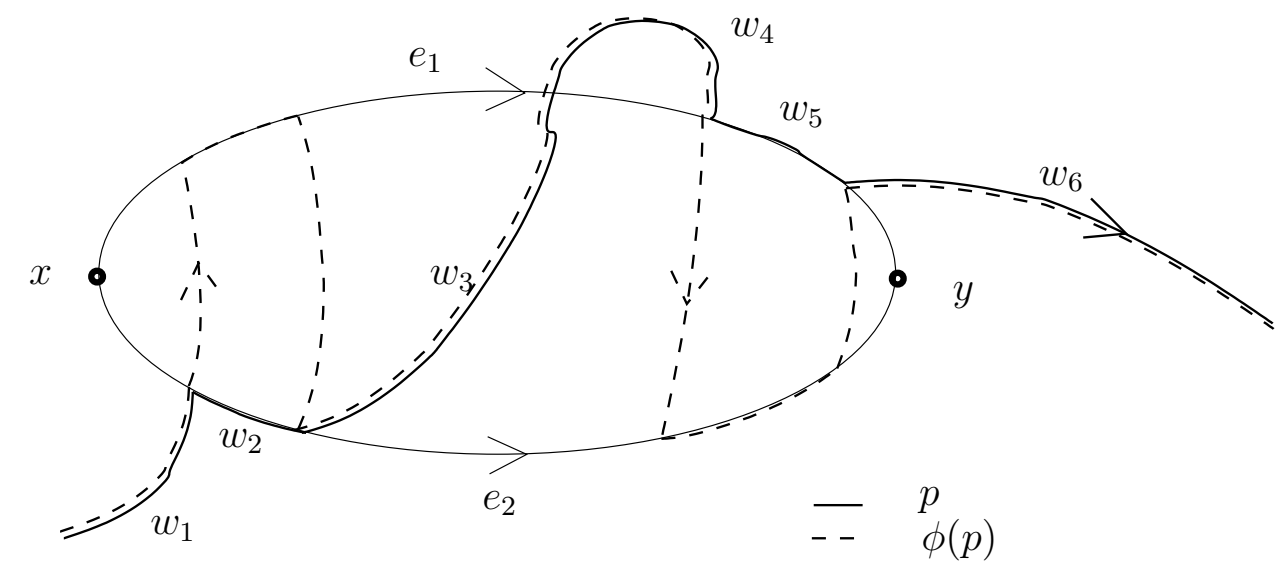

Figure 3.1: The action of an edge-interchanger. The solid line shows some path $p$, the dashed line shows $\phi(p)$.

Analogous relations hold in the case that $w_{k}=\left[\left[\left[c_{2}^{t_{1}, t_{2}}\right]\right]\right]$.

Let now $w_{k}$ be such that $r\left(w_{k}\right) \cap r\left(e_{1,2}\right) \subset\left\{s\left(w_{k}\right), t\left(w_{k}\right)\right\}$, and $w_{k}=w_{l}^{\prime} \circ w_{l+l^{\prime}}^{\prime}$. Then obviously also $r\left(w_{l+j}^{\prime}\right) \cap r\left(e_{1,2}\right) \subset\left\{s\left(w_{l+j}^{\prime}\right), t\left(w_{l+j}^{\prime}\right)\right\}$ for all $j \in\left\{1, \ldots, l^{\prime}\right\}$. By (3.8) we immediately see that

$$
\tilde{\phi}\left(w_{l}^{\prime}\right) \circ \ldots \circ \tilde{\phi}\left(w_{l+l^{\prime}}^{\prime}\right)=\tilde{\phi}\left(w_{k}\right) .
$$

Since these are the only cases occurring, we conclude that $\tilde{\phi}$ is a well-defined map of morphisms in $\operatorname{Mor}_{\tilde{\mathcal{C}}}\left(z, z^{\prime}\right)$ to morphisms in $\operatorname{Mor}_{\mathcal{P}}\left(z, z^{\prime}\right)$ for all $z, z^{\prime} \in \Sigma$.

The properties of a functor remain to be shown. But given two morphisms $v, w$ in $\tilde{\mathcal{C}}$ such that $t(v)=s(w)$, and decompositions $v=v_{1} \circ \ldots \circ v_{n}$ and $w=w_{1} \circ \ldots \circ w_{m}$ w.r.t. $e_{1}$ and $e_{2}$, then $v \circ w=v_{1} \circ \ldots \circ v_{n} \circ w_{1} \circ \ldots \circ w_{m}$ is a decomposition of $v \circ w$ w.r.t. $e_{1}$ and $e_{2}$. By (3.7) we see that

$$
\tilde{\phi}(v \circ w)=\tilde{\phi}(v) \circ \tilde{\phi}(w) .
$$

Since $\phi$ leaves beginning- and endpoints of paths invariant, we conclude that the map induces a functor $\tilde{\phi}: \tilde{\mathcal{C}} \rightarrow \mathcal{P}$ that acts trivially on the objects $|\tilde{\mathcal{C}}|=|\mathcal{P}|=\Sigma$.

Lemma 3.2 For $v, w$ morphisms in $\tilde{\mathcal{C}}$ with $[[v]]=[[w]]$, one has $\tilde{\phi}(v)=\tilde{\phi}(w)$. Thus, $\tilde{\phi}$ descends to a functor $\phi: \mathcal{P} \rightarrow \mathcal{P}$. Furthermore, one has $\phi^{2}=\mathrm{id}_{\mathcal{P}}$, i.e. $\phi \in \mathcal{A} u t(\mathcal{P})$.

Proof: If $[[v]]=[[w]]$, then by definition one can reach $w$ by starting with $v$ and deleting or inserting ways $u \circ u^{-1}$. Hence we need to show $\tilde{\phi}\left(w^{-1}\right)=\tilde{\phi}(w)^{-1}$. Since for ways $w, v$ in $\tilde{\mathcal{C}}$ one has $(v \circ w)^{-1}=w^{-1} \circ v^{-1}$, we have only to show

\footnotetext{
${ }^{5}$ This only holds if $t_{1}<t_{2}$. If $t_{1}>t_{2}$ then the corresponding points have the property that $t_{1}=t_{l}>$ $t_{l+1}>\ldots>t_{l+l^{\prime}+1}=t_{2}$. The proof is then analogous.
} 
$\tilde{\phi}\left(w^{-1}\right)=\tilde{\phi}(w)^{-1}$ for $w$ being of one of the types (3.8). But for $w=\left[c_{1}^{t_{1}, t_{2}}\right]$ this follows from the fact that

$$
\left[c_{1}^{t_{1}, t_{2}}\right]^{-1}=\left[\left(c_{1}^{t_{1}, t_{2}}\right)^{-1}\right]=\left[c_{1}^{t_{2}, t_{1}}\right]
$$

with an analogous relation for $c_{2}^{t_{1}, t_{2}}$. For $w$ touching the edges $e_{1}$ and $e_{2}$ at most at its beginning- and endpoint, the assertion is trivial. We thus get $\tilde{\phi}(v)=\tilde{\phi}(w)$ for $[[w]]=[[v]]$. Thus, $\tilde{\phi}$ descends to a functor $\phi: \mathcal{P} \rightarrow \mathcal{P}$.

It remains to show that $\phi^{2}=\mathrm{id}_{\mathcal{P}}$. Take a path $p$ in $\mathcal{P}$. Choose a representative $w$ in $\tilde{\mathcal{C}}$, i.e. $p=[[w]]$. Then $\phi(p)=\tilde{\phi}(w)$. Decompose $w$ w.r.t the edges $e_{1}, e_{2}$ :

$$
w=w_{1} \circ \ldots \circ w_{n}
$$

where $w_{k}$ are of the type (3.8). Assume $w_{k}$ to meet the edges $e_{1}$ and $e_{2}$ at most at their endpoints. Then

$$
\phi^{2}\left(\left[\left[w_{k}\right]\right]\right)=\phi\left(\tilde{\phi}\left(w_{k}\right)\right)=\left[\left[w_{k}\right]\right] .
$$

Let now $w_{k}=\left[c_{1}^{t_{1}, t_{2}}\right]$. Then

$$
\tilde{\phi}\left(w_{k}\right)=p_{t_{1}} \circ\left[c_{2}^{t_{1}, t_{2}}\right] \circ p_{t_{2}}^{-1}
$$

By construction, the $p_{t}$ are such that they have a representative in $\tilde{\mathcal{C}}$ which touches the edges $e_{1}, e_{2}$ only at their beginning- and endpoints $s\left(p_{t}\right), t\left(p_{t}\right)$. It follows that $\phi\left(p_{t}\right)=p_{t}$ for all $t \in[0,1]$. So we have

$$
\begin{aligned}
\phi^{2}\left(\left[\left[w_{k}\right]\right]\right) & =\phi\left(p_{t_{1}} \circ\left[\left[\left[c_{2}^{t_{1}, t_{2}}\right]\right]\right] \circ p_{t_{2}}^{-1}\right) \\
& =p_{t_{1}} \circ p_{t_{1}}^{-1} \circ\left[\left[\left[c_{1}^{t_{1}, t_{2}}\right]\right]\right] \circ p_{t_{2}} \circ p_{t_{2}}^{-1} \\
& =\left[\left[\left[c_{1}^{t_{1}, t_{2}}\right]\right]\right]=\left[\left[w_{k}\right]\right] .
\end{aligned}
$$

Analogously for $w_{k}=\left[c_{2}^{t_{1}, t_{2}}\right]$. We conclude that $\phi^{2}=\operatorname{id}_{\mathcal{P}}$. So $\phi \in \mathcal{A} u t(\mathcal{P})$.

From the definition is immediate that $\phi\left(e_{1}\right)=e_{2}$ and vice versa. This justifies the name "edge-interchanger", although not only edges can be interchanged, but every two paths without self-intersection that do not self-intersect. Note that $\phi$ is an automorphism that acts trivially on the points in $\Sigma$, but nontrivially on the paths. Every path, however, that is composed of edges that meet $e_{1}, e_{2}$ in at most finitely many points is left invariant under $\phi$. Note further that $\phi$ depends on a chosen parametrization of $e_{1}, e_{2}$, as well as a choice $\left\{p_{t}\right\}_{t \in(0,1)}$. This shows that there are many such automorphisms that interchange $e_{1}$ and $e_{2}$. 


\section{Unitarity of automorphisms}

In section 2.3 we have seen that the set of metagraphs is suitable for addressing topological questions on $\overline{\mathcal{A}}$. However, for the measure theory on $\overline{\mathcal{A}}$, the concept of metagraphs is too broad: Not every metagraph $\mu \in \mathcal{M}$ has the property that

$$
\left(\pi_{\mu}\right)_{*} \mu_{A L}=\mu_{H}
$$

The metagraphs that have this property are called primitive metagraphs. But since this definition rests on analytical terms, it is a priori not clear whether the automorphisms preserve the set of primitive metagraphs. The proof for this will be delivered in this section. As an immediate consequence, the operators on $\mathcal{H}_{\text {kin }}$ induced by elements $\mathcal{A} u t(\mathcal{P})$ will all be unitary.

For the abelian gauge group $G=U(1)$ this is quite easy to see, and we will give the short proof for this, before we will turn to the general case and prove the assertion for general gauge group $G$.

\subsection{The kinematical Hilbert space}

In this section we review the kinematical Hilbert space $\mathcal{H}_{\text {kin }}$ for general gauge groups $G$, and discuss the action of the automorphisms on it.

Definition 4.1 Let $\mu=\left\{p_{1}, \ldots, p_{E}\right\}$ be a metagraph. Then a function $f: \overline{\mathcal{A}} \rightarrow \mathbb{C}$ is called cylindrical over $\mu$, iff there is a function $F: G^{E} \rightarrow \mathbb{C}$ such that

$$
f(A)=F\left(A\left(p_{1}\right), \ldots, A\left(p_{E}\right)\right) .
$$

If $F$ is continuous (differentiable, smooth) then $f$ is called a continuous (differentiable, smooth) cylindrical function over $\mu$. The set of all smooth cylindrical functions over $\mu$ is denoted by $\operatorname{Cyl}(\mu)$. The set of all smooth cylindrical functions is denoted by Cyl.

Obviously, if $f \in C y l(\mu)$, then $f \in C y l\left(\mu^{\prime}\right)$ for all metagraphs $\mu^{\prime}$ such that $\mu \leq \mu^{\prime}$. Thus, every cylindrical function is cylindrical over a graph. The advantage of the cylindrical functions lies in the following lemma.

Lemma 4.1 The set of cylindrical functions $C y l$ is a dense subspace in the set of all continuous functions $C(\overline{\mathcal{A}})$, and hence also in $\mathcal{H}_{\mathrm{kin}}=L^{2}\left(\overline{\mathcal{A}}, d \mu_{A L}\right)$.

In the analytical category, the concept of graphs provides an orthonormal basis of $\mathcal{H}_{\text {kin }}$.

Lemma 4.2 Let $G$ be a semisimple, compact and connected gauge group. Let $\gamma=\left\{e_{1}, \ldots, e_{E}\right\}$ be a minimal graph, i.e. there is no other graph $\tilde{\gamma} \leq \gamma$. Let furthermore $\left\{\pi_{k}\right\}_{k=1}^{E}$ be a sequence of irreducible representations of $G$, and $m_{k}, n_{k} \in$ $\left\{1, \ldots, \operatorname{dim} \pi_{k}\right\}$ be numbers. Then the function

$$
T_{\gamma, \vec{\pi}, \vec{n}, \vec{m}}(A):=\prod_{k=1}^{E} \sqrt{\operatorname{dim} \pi_{k}} \pi_{k}\left(A\left(e_{k}\right)\right)_{n_{k} m_{k}}
$$

is cylindrical over $\gamma$. The set of all these functions provide an orthonormal basis for $\mathcal{H}_{\text {kin }}$. 
In the case of $G=S U(2)$, the corresponding functions (4.3) are called spin-network functions (SNF), and are denoted by $T_{\gamma, \vec{j}, \vec{n}, \vec{m}}$, where each of the $j_{k}$ is a half-integer corresponding to an irreducible representation of $S U(2)$, and $m_{k}, n_{k} \in\{-j,-j+$ $1, \ldots, j\}$. In the case of $G=U(1)$, where all representations are one-dimensional, the functions $T_{\gamma, \vec{n}}$ are called charge-network functions, and the $n_{k} \in \mathbb{Z}$ denote irreducible representations of $U(1)$.

Lemma 4.3 Let $\phi \in \mathcal{A} u t(\mathcal{P})$ be an automorphism of the path groupoid, and $T_{\gamma, \vec{n}}$ a charge-network function. Then the function

$$
\left(\hat{U}(\phi) T_{\gamma, \vec{n}}\right)(A):=T_{\gamma, \vec{n}}\left(\alpha_{\phi} A\right)
$$

is again a charge-network function.

Proof: If $\gamma$ is a minimal graph, then $\phi(\gamma)$ is not necessarily one, only a metagraph. But there is a minimal graph $\gamma^{\prime}=\left\{e_{1}^{\prime}, \ldots, e_{E^{\prime}}^{\prime}\right\}$ such that $\phi(\gamma) \leq \gamma^{\prime}$. In particular

$$
\phi\left(e_{k}\right)=e_{l_{k}^{1}}^{\prime}, \circ \ldots, \circ e_{l_{k}^{m_{k}}}^{\prime}
$$

for each $k \in\{1, \ldots, E\}$, and the $e_{l}^{\prime}$ are edges (or their inverses) in $\gamma^{\prime}$. With

$$
T_{\gamma, \vec{n}}(A)=\prod_{k=1}^{E} A\left(e_{k}\right)^{n_{k}}
$$

we get

$$
\begin{aligned}
\left(\hat{U}(\phi) T_{\gamma, \vec{n}}\right)(A) & =T_{\gamma, \vec{n}}\left(\alpha_{\phi} A\right) \\
& =\prod_{k=1}^{E} A\left(e_{l_{k}^{1}}^{\prime}, \circ \ldots, \circ e_{l_{k}^{m_{k}}}^{\prime}\right)^{n_{k}} .
\end{aligned}
$$

But using the functorial properties of $A$, we get a product of $A\left(e_{l_{k}^{m}}^{\prime}\right)^{n_{k}}$, which we can - due to the abelianess of $U(1)$ - group together to obtain

$$
T_{\gamma, \vec{n}}\left(\alpha_{\phi} A\right)=\prod_{l=1}^{E^{\prime}} A\left(e_{l}^{\prime}\right)^{m_{l}}=T_{\gamma^{\prime}, \vec{m}}(A) .
$$

So the automorphism map charge-network functions into charge-network functions.

Corollary 4.1 Let $\phi \in \mathcal{A} u t(\mathcal{P})$ be an automorphism and $\mathcal{H}_{\text {kin }}$ be the kinematical Hilbert space for the gauge group $G=U(1)$. Then the operator

$$
(\hat{U}(\phi) f):=f\left(\alpha_{\phi} A\right)
$$

for any function $f \in \mathcal{H}_{\text {kin }}$ is unitary. 
Proof: By lemma 4.3 the map (4.9) is defined on the charge-network functions $T_{\gamma, \vec{n}}$, and map this set into itself. Furthermore, the vector $\Omega$, corresponding to the constant function 1 on $\overline{\mathcal{A}}$, which can be written as $T_{\gamma, \overrightarrow{0}}$ for any graph $\gamma$, is trivially left invariant by the $\phi \in \mathcal{A} u t(\mathcal{P})$. Let now $\gamma$ be a minimal graph and $f \in \operatorname{Cyl}(\gamma)$ a smooth cylindrical function over $\gamma$. Then

$$
f=\sum_{\vec{n} \in \mathbb{Z}^{E}} c_{\vec{n}} T_{\gamma, \vec{n}}
$$

One has, by definition of $\mu_{A L}$ and the orthonormality of the charge-network functions:

$$
\int_{\overline{\mathcal{A}}} d \mu_{A L}(A) f(A)=c_{\overrightarrow{0}}
$$

On the other hand:

$$
\int_{\overline{\mathcal{A}}} d \mu_{A L}(A) f\left(\alpha_{\phi} A\right)=\sum_{\vec{n} \in \mathbb{Z}} c_{\vec{n}} \int_{\overline{\mathcal{A}}} d \mu_{A L}(A) T_{\gamma, \vec{n}}\left(\alpha_{\phi} A\right)
$$

But if $T_{\gamma, \vec{n}} \neq T_{\gamma, \overrightarrow{0}}$, then also $\hat{U}(\phi) T_{\gamma, \vec{n}} \neq T_{\gamma^{\prime}, \overrightarrow{0}}$, since no not constant function on $\overline{\mathcal{A}}$ can be mapped to the constant function on $\overline{\mathcal{A}}$. Thus, since the integral over $\overline{\mathcal{A}}$ of all not constant charge-network functions is zero, we get

$$
\sum_{\vec{n} \in \mathbb{Z}} c_{\vec{n}} \int_{\overline{\mathcal{A}}} d \mu_{A L}(A) T_{\gamma, \vec{n}}\left(\alpha_{\phi} A\right)=c_{\overrightarrow{0}}=\int_{\overline{\mathcal{A}}} d \mu_{A L}(A) f(A)
$$

So we have

$$
\int_{\overline{\mathcal{A}}} d \mu_{A L}(A) f\left(\alpha_{\phi} A\right)=\int_{\overline{\mathcal{A}}} d \mu_{A L}(A) f(A)
$$

for all functions $f \in C y l$. But this is a set dense in the continuous functions w.r.t the supremum norm on $C(\overline{\mathcal{A}})$, and since $\alpha_{\phi}: \overline{\mathcal{A}} \rightarrow \overline{\mathcal{A}}$ is continuous, we conclude that (4.14) holds for all continuous functions $f \in C(\overline{\mathcal{A}})$. Hence

$$
\left(\alpha_{\phi}\right)^{*} \mu_{A L}=\mu_{A L}
$$

i.e. the integration measure os preserved under the action of $\phi \in \mathcal{A} u t(\mathcal{P})$. Consequently, $\hat{U}(\phi)$ defined by $(4.9)$ is a unitary map on $\mathcal{H}_{\text {kin }}=L^{2}\left(\overline{\mathcal{A}}, d \mu_{A L}\right)$.

It should be noted that the key for establishing this short proof is the fact that the orthonormal basis elements $T_{\gamma, \vec{n}}$ are mapped into themselves by the action of $\mathcal{A} u t(\mathcal{P})$. This rested on the fact that $G=U(1)$ is abelian, and is not true for nonabelian gauge groups, such as $G=S U(2)$. In these cases, the automorphisms also act unitarily on the corresponding $\mathcal{H}_{\text {kin }}$, however the proof is much more involved, as we will see in the following section. 


\subsection{Unitarity of Automorphisms}

In the last section, we have seen that in the case of $G=U(1)$ it is quite straightforward to show that the action of the automorphisms $\mathcal{A} u t(\mathcal{P})$ on $\mathcal{H}_{\text {kin }}$ is unitary. However, this proof rested crucially on the abelianess of $U(1)$. In the following, we will prove unitarity for gauge group $G=S U(2)$.

For showing unitarity for the automorphisms $\mathcal{A} u t(\mathcal{P})$ in the case $G=S U(2)$, we will make explicit use of the natural transformations of the identity $\phi_{b, p} \in \mathcal{N}$ defined in section 3.1

In this section we will prove that all natural transformations of the identity $\phi_{b, p} \in \mathcal{N}$ act unitarily on $\mathcal{H}_{\text {kin }}$. The basic idea of the proof is as follows: Since $\mathcal{N}$ is a group, every $\phi_{b, p}$ can be written as a sequence of other elements $\phi_{b, p}=$ $\phi_{b_{N}, p_{N}} \circ \ldots \circ \phi_{b_{1}, p_{1}}$, each of which are not too different from the identity. For these we will be able to prove that they act unitarily, because they do not change a graph much, hence can be controlled.

Lemma 4.4 Let $\gamma=\left\{e_{1}, \ldots, e_{E}\right\}$ be a graph. Let furthermore $v \in V(\gamma)$ be a vertex in the graph, and $e_{k_{1}} \circ \ldots \circ e_{k_{n}}$ be a path in $\gamma$ with the following properties:

- All the $e_{k_{l}}$ are edges in $\gamma$.

- All the $e_{k_{l}}$ are distinct.

- $s\left(e_{k_{1}}\right) \neq t\left(e_{k_{n}}\right)$.

Then the natural transformation of the identity $\phi_{b, p}$ defined by

$$
\begin{aligned}
b\left(s\left(e_{k_{1}}\right)\right) & :=t\left(e_{k_{n}}\right), \quad b\left(t\left(e_{k_{n}}\right)\right):=s\left(e_{k_{1}}\right), \quad b(x):=x \text { else } \\
p_{t\left(e_{k_{n}}\right)} & :=\left(e_{k_{1}} \circ \ldots \circ e_{k_{n}}\right)^{-1}, \quad p_{s\left(e_{k_{1}}\right)}:=e_{k_{1}} \circ \ldots \circ e_{k_{n}}, \quad p_{x}:=\mathrm{id}_{x} \text { else }
\end{aligned}
$$

acts unitarily on $\mathcal{H}_{\gamma}$.

Proof: First we note that $\phi_{b, p}$ maps $\mathcal{H}_{\gamma}$ to itself. Since the definition of $\phi_{b, p}$ is such that $\left(\phi_{b, p}\right)^{2}=\mathrm{id}_{\mathcal{P}}$ is the identity functor, so does its inverse. Thus, every edge $e_{k}$ is mapped to a combination of edges:

$$
\phi_{b, p}\left(e_{k}\right)=\vartheta_{k}\left(e_{1}, \ldots, e_{E}\right)
$$

where the $\vartheta_{k}\left(e_{1}, \ldots, e_{E}\right)$ is a word of the $\left\{e_{1}, \ldots, e_{E}\right\}$, i.e. a product of edges and their inverses. Thus, $\phi_{b, p}$ induces an endomorphism $\phi$ on the free group $F_{E}$ in the $E$ letters $\left\{e_{1}, \ldots e_{E}\right\}$ (which is defined by $\phi\left(e_{k}\right)=\vartheta_{k}\left(e_{1}, \ldots, e_{E}\right)$ ). But since its inverse also does, $\phi$ is invertible (it is in fact its own inverse), and hence defines an automorphism on $F_{E}$. By corollary B.1, we have

$$
\begin{aligned}
\int_{S U(2)^{E}} d \mu_{H} & \left(h_{1}, \ldots, h_{E}\right) F\left(h_{1}, \ldots, h_{E}\right) \\
& =\int_{S U(2)^{E}} d \mu_{H}\left(h_{1}, \ldots, h_{E}\right) F\left(\vartheta_{1}\left(h_{1}, \ldots, h_{E}\right), \ldots, \vartheta_{E}\left(h_{1}, \ldots, h_{E}\right)\right),
\end{aligned}
$$


and hence

$$
\int_{\overline{\mathcal{A}}} d \mu_{A L}(A) f(A)=\int_{\overline{\mathcal{A}}} d \mu_{A L}(A) \alpha_{\phi_{b, p}} f(A) .
$$

Since $f$ is cylindrical over $\gamma$, the immediate conclusion from (4.18) is that $\phi_{b, p}$ acts unitarily on $\mathcal{H}_{\gamma}$.

For $f$ being cylindrical over a graph $\gamma$, and $\phi_{b, p} \in \mathcal{N}$ be a natural transformation of the identity functor, $\phi_{b, p}$ will generically not satisfy the conditions of lemma 4.4 , but it can be composed of a sequence of elements in $\mathcal{N}$ that do, which will be the key part of the following lemma.

Lemma 4.5 Let $f$ be a function cylindrical over a graph $\gamma$, and $\phi_{b, p}$ a natural transformation of the identity. Then

$$
\int_{\overline{\mathcal{A}}} d \mu_{A L}(A) f(A)=\int_{\overline{\mathcal{A}}} d \mu_{A L}(A) \alpha_{\phi_{b, p}} f(A) .
$$

Proof: Let $e_{1}, \ldots, e_{E}$ be the edges of the graph $\gamma$, and $v_{1}, \ldots v_{V}$ be the vertices. Without loss of generality, we can decompose the edges which are loops, such that their beginning- and endpoints do not coincide. Consider the paths $p_{v_{1}}, \ldots, p_{v_{V}}$ which enter the definition of $\phi_{b, p}$. Now decompose the $e_{k}$ and the $p_{v_{l}}$ into smaller edges $e_{1}^{\prime}, \ldots, e_{E^{\prime}}^{\prime}$ that form a graph $\gamma^{\prime}$, such that all the $e_{k}$ and the $p_{v_{l}}$ can be composed from edges in $\gamma^{\prime}$ (and their inverses). Now decompose the edges $e_{k}^{\prime}$ of $\gamma^{\prime}$ further into edges $e_{k}^{\prime \prime}$ (forming a graph $\gamma^{\prime \prime}$ ), such that the following holds: Each path $p_{v_{l}}$ can be decomposed into a product of $N$ paths $\tilde{e}_{k}$

$$
p_{v_{l}}=\tilde{e}_{1}^{l} \circ \tilde{e}_{2}^{l} \circ \ldots \tilde{e}_{N}^{l}
$$

such that

- Each $\tilde{e}_{m}^{l}, l \in\{1, \ldots, V\}, m \in\{1, \ldots, N\}$ is a sequence of distinct edges $e_{k}^{\prime \prime}$ (or their inverses) in $\gamma^{\prime \prime}$

- For each $m \in\{1, \ldots, N\}$ the $2 V$ points $s\left(\tilde{e}_{m}^{l}\right)$ and $t\left(\tilde{e}_{m}^{l}\right)$ for all $l \in\{1, \ldots, V\}$ are distinct.

Note that this decomposition is possible, even if some $p_{v_{l}}$ are the constant paths: In this case, the paths in (4.20) cancel each other out such that each $\tilde{e}_{1}^{l} \circ \tilde{e}_{2}^{l} \circ \ldots \tilde{e}_{m}^{l}$ is nontrivial, while $\tilde{e}_{1}^{l} \circ \tilde{e}_{2}^{l} \circ \ldots \tilde{e}_{N}^{l}$ is a constant path again.

Then we define a natural transformation of the identity $\phi_{b_{m}^{l}, p_{m}^{l}}$ by the following data:

$$
\begin{aligned}
& b_{m}^{l}\left(s\left(\tilde{e}_{m}^{l}\right)\right)=t\left(\tilde{e}_{m}^{l}\right), \quad b_{m}^{l}\left(t\left(\tilde{e}_{m}^{l}\right)\right)=s\left(\tilde{e}_{m}^{l}\right), \quad b_{m}^{l}(x)=x \text { else } \\
&\left(p_{m}^{l}\right)_{s\left(\tilde{e}_{m}^{l}\right)}=\tilde{e}_{m}^{l}, \quad\left(p_{m}^{l}\right)_{t\left(\tilde{e}_{m}^{l}\right)}=\left(\tilde{e}_{m}^{l}\right)^{-1}
\end{aligned}
$$

This defines an element in $\mathcal{N}$, as one can readily see. Furthermore, we can see that, for one fixed $m \in\{1, \ldots, N\}$, the $\phi_{b_{m}^{l}, p_{m}^{l}}$ for all $l \in\{1, \ldots, V\}$ commute, due to the condition that all the $\tilde{e}_{m}^{l}$ have distinct beginning- and endpoints. Now define 


$$
\begin{aligned}
\phi_{\tilde{b}, \tilde{p}}:=\phi_{b_{N}^{V}, p_{N}^{V}} & \circ \phi_{b_{N}^{V-1}, p_{N}^{V-1}} \circ \ldots \circ \phi_{b_{N}^{1}, p_{N}^{1}} \circ \phi_{b_{N-1}^{V}, p_{N-1}^{V}} \circ \ldots \\
& \circ \phi_{b_{N-1}^{1}, p_{N-1}^{1}} \circ \phi_{b_{N-2}^{V}, p_{N-2}^{V}} \circ \ldots \circ \phi_{b_{1}^{1}, p_{1}^{1}}
\end{aligned}
$$

Now let $e_{k}$ be an edge in $\gamma$, with starting point $v_{l^{\prime}}$ and ending point $v_{l^{\prime \prime}}$. Then for every $m \in\{1, \ldots, N\}$ we have

$$
\begin{gathered}
\phi_{b_{m}^{V}, p_{m}^{V}} \circ \ldots \circ \phi_{b_{m}^{1}, p_{m}^{1}}\left(\left(\tilde{e}_{m-1}^{l^{\prime}}\right)^{-1} \circ \ldots \circ\left(\tilde{e}_{1}^{l^{\prime}}\right)^{-1} \circ e_{k} \circ \tilde{e}_{1}^{l^{\prime \prime}} \circ \ldots \circ \tilde{e}_{m-1}^{l^{\prime \prime}}\right) \\
=\left(\tilde{e}_{m}^{l^{\prime}}\right)^{-1} \circ \ldots \circ\left(\tilde{e}_{1}^{l^{\prime}}\right)^{-1} \circ e_{k} \circ \tilde{e}_{1}^{l^{\prime \prime}} \ldots \circ \tilde{e}_{m}^{l^{\prime \prime}} .
\end{gathered}
$$

In order to show this, we first note that, since all the $\phi_{b_{m}^{l}, p_{m}^{l}}$ commute for all $l \in\{1, \ldots, V\}$ by construction, we can move $\phi_{b_{m}^{\prime}, p_{m}^{l^{\prime}}}$ and $\phi_{b_{m}^{l^{\prime \prime}}, p_{m}^{l^{\prime \prime}}}$ to the left in (4.22). Since all the starting- and endpoints of the $\tilde{e}_{m}^{l}$ are different from $s\left(\tilde{e}_{m}^{l^{\prime}}\right)$ and $s\left(\tilde{e}_{m}^{l^{\prime \prime}}\right)$ by construction, the action of the $\phi_{b_{m}^{l}, p_{m}^{l}}$ on $\left(\tilde{e}_{m-1}^{l^{\prime}}\right)^{-1} \circ \ldots \circ\left(\tilde{e}_{1}^{l^{\prime}}\right)^{-1} \circ e_{k} \circ \tilde{e}_{1}^{l^{\prime \prime}} \circ \ldots \circ \tilde{e}_{m-1}^{l^{\prime \prime}}$ for $l \neq l^{\prime}, l^{\prime \prime}$ is trivial. Thus we get

$$
\begin{aligned}
& \phi_{b_{m}^{V}, p_{m}^{V}} \circ \ldots \circ \phi_{b_{m}^{1}, p_{m}^{1}}\left(\left(\tilde{e}_{m-1}^{l^{\prime}}\right)^{-1} \circ \ldots \circ\left(\tilde{e}_{1}^{l^{\prime}}\right)^{-1} \circ e_{k} \circ \tilde{e}_{1}^{l^{\prime \prime}} \circ \ldots \circ \tilde{e}_{m-1}^{l^{\prime \prime}}\right)
\end{aligned}
$$

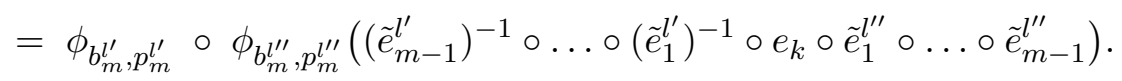

But starting- and endpoint of $\tilde{e}_{m}^{l^{\prime}}$ and $\tilde{e}_{m}^{l^{\prime \prime}}$ are all distinct, and hence, by definition of the $\phi_{b_{m}^{l}, p_{m}^{l}}$, we get.

$$
\begin{aligned}
& \phi_{b_{m}^{l^{\prime}}, p_{m}^{l^{\prime}}} \circ \phi_{b_{m}^{l^{\prime \prime}}, p_{m}^{l^{\prime \prime}}}\left(\left(\tilde{e}_{m-1}^{l^{\prime}}\right)^{-1} \circ \ldots \circ\left(\tilde{e}_{1}^{l^{\prime}}\right)^{-1} \circ e_{k} \circ \tilde{e}_{1}^{l^{\prime \prime}} \circ \ldots \circ \tilde{e}_{m-1}^{l^{\prime \prime}}\right) \\
& =\left(\tilde{e}_{m}^{l^{\prime}}\right)^{-1} \circ \ldots \circ\left(\tilde{e}_{1}^{l^{\prime}}\right)^{-1} \circ e_{k} \circ \tilde{e}_{1}^{l^{\prime \prime}} \circ \ldots \circ \tilde{e}_{m}^{l^{\prime \prime}} \text {. }
\end{aligned}
$$

An immediate consequence from (4.22), together with (4.21), is that

$$
\phi_{b, p}\left(e_{k}\right)=\phi_{\tilde{b}, \tilde{p}}\left(e_{k}\right)
$$

for all edges $e_{k}$ in the graph $\gamma$. But $\phi_{\tilde{b}, \tilde{p}}$ is, by (4.21) a product of natural transformations of the identity $\phi_{b_{m}^{l}, p_{m}^{l}}$, all of which satisfy the conditions of Lemma 4.4. Thus, we get

$$
\int_{\overline{\mathcal{A}}} d \mu_{A L}(A) \alpha_{\phi_{b, p}} f(A)=\int_{\overline{\mathcal{A}}} d \mu_{A L}(A) \alpha_{\phi_{\tilde{b}, \tilde{p}}} f(A)=\int_{\overline{\mathcal{A}}} d \mu_{A L}(A) f(A)
$$

for all functions $f$ cylindrical over $\gamma$. This finishes the proof.

Corollary 4.2 All natural transformations of the identity $\phi_{b, p} \in \mathcal{N}$ act unitarily on $\mathcal{H}_{\text {kin }}$.

Proof: From lemma 4.5 we get that

$$
\int_{\overline{\mathcal{A}}} d \mu_{A L}(A) \alpha_{\phi_{b, p}} f(A)=\int_{\overline{\mathcal{A}}} d \mu_{A L}(A) f(A)
$$


holds for all functions $f \in C y l$. Thus, $\alpha_{\phi_{b, p}}$ induces an operator on $\mathcal{H}_{\text {kin }}$ which is isometric on a dense subspace. Thus, it is also unitary on all of $\mathcal{H}_{\text {kin }}$. So, all natural transformations of the identity $\phi_{b, p} \in \mathcal{N}$ act unitarily on $\mathcal{H}_{\text {kin }}$.

With this, we can now make the next step in the proof that all automorphisms act unitarily on $\mathcal{H}_{\text {kin }}$.

Lemma 4.6 Let $e$ be an edge which is no loop, i.e. $s(e) \neq t(e)$. Let $f \in C y l(e)$ be a function cylindrical over $e$. Then, for any automorphism $\phi \in \mathcal{A} u t(\mathcal{P})$, one has

$$
\int_{\overline{\mathcal{A}}} d \mu_{A L}(A) \alpha_{\phi} f(A)=\int_{\overline{\mathcal{A}}} d \mu_{A L}(A) f(A)
$$

Proof: Let $p=\phi(e)$. Since $s(e) \neq t(e)$, also $s(p) \neq t(p)$. Now decompose $p$ into edges $p=e_{1} \circ \ldots \circ e_{E}$. If necessary, decompose the edges $e_{k}$ further, until $s\left(e_{1}\right), t\left(e_{1}\right)$ and $t\left(e_{E}\right)$ are all distinct. Then define the natural transformation of the identity $\phi_{b, p}$ by

$$
\begin{array}{r}
b\left(t\left(e_{1}\right)\right):=t\left(e_{E}\right), \quad b\left(t\left(e_{E}\right)\right):=t\left(e_{1}\right), \quad b(x)=x \text { else }(4 \\
p_{t\left(e_{1}\right)}:=e_{2} \circ \ldots \circ e_{E}, \quad p_{t\left(e_{E}\right)}:=e_{E}^{-1} \circ \ldots \circ e_{2}^{-1}, \quad p_{x}=\mathrm{id}_{x} \text { else. }
\end{array}
$$

Since $f$ is cylindrical over the edge $e$, there is a function $F$ such that

$$
\int_{\overline{\mathcal{A}}} d \mu_{A L}(A) f(A)=\int_{S U(2)} d \mu_{H}(h) F(h) .
$$

But obviously $\alpha_{\phi_{b, p}} \circ \alpha_{\phi} f$ is cylindrical over the edge $e_{1}$. Since $\phi_{b, p}(\phi(e))=e_{1}$, we have

$$
\int_{\overline{\mathcal{A}}} d \mu_{A L}(A) \alpha_{\phi_{b, p} \circ \phi} f(A)=\int_{S U(2)} d \mu_{H}(h) F(h),
$$

so

$$
\int_{\overline{\mathcal{A}}} d \mu_{A L}(A) f(A)=\int_{\overline{\mathcal{A}}} d \mu_{A L}(A) \alpha_{\phi_{b, p}} \circ \alpha_{\phi} f(A) .
$$

But since all natural transformations of the identity $\phi_{b, p} \in \mathcal{N}$ leave the AshtekarIsham-Lewandowski measure invariant by lemma (4.5), we have

$$
\int_{\overline{\mathcal{A}}} d \mu_{A L}(A) f(A)=\int_{\overline{\mathcal{A}}} d \mu_{A L}(A) \alpha_{\phi} f(A),
$$

which was the claim.

Lemma 4.6 shows that for $f, g$ being cylindrical over an edge $e$, one has

$$
\langle\hat{U}(\phi) f \mid \hat{U}(\phi) g\rangle=\langle f \mid g\rangle \text {. }
$$

In fact, in the proof of lemma 4.6 we have shown that every path $p$ with $s(p) \neq t(p)$ can be mapped to an edge by an automorphism (in fact, by an element of $\mathcal{N}$ ), hence (4.34) holds also for functions $f, g \in C y l(p)$. In the following we will look at arbitrary automorphisms $\phi \in \mathcal{A} u t(\mathcal{P})$, and use their properties as maps from $\overline{\mathcal{A}}$ to $\overline{\mathcal{A}}$, as well as (4.34) in order to show that they act unitarily on $\mathcal{H}_{\text {kin }}$. 
Lemma 4.7 Let $\phi \in \mathcal{A} u t(\mathcal{P})$ and $\hat{U}(\phi)$ be the induced operator on $\mathcal{H}_{\text {kin }}$, and $\hat{U}(\phi)^{\dagger}$ be its adjoint. Define $\hat{I}_{\phi}:=\hat{U}(\phi)^{\dagger} \hat{U}(\phi)$, then there is a sequence of continuous functions $f_{n} \in C(\overline{\mathcal{A}})$ such that

$$
\lim _{n \rightarrow \infty} \hat{a}_{f_{n}}=\hat{I}_{\phi}
$$

converges in the weak operator topology, where for a continuous function $f \in C(\overline{\mathcal{A}})$ the operator $\hat{a}_{f}$ denotes multiplication with $f$ on $\mathcal{H}_{\text {kin }}$.

Proof: Let $f \in C(\overline{\mathcal{A}})$ be a continuous function on $\overline{\mathcal{A}}$. Then $\alpha_{\phi} f$ is also continuous. Denote with $\bar{f}$ the complex conjugate function, then $\alpha_{\phi} \bar{f}=\overline{\alpha_{\phi} f}$. Since $\hat{U}(\phi) \hat{a}_{f} \hat{U}(\phi)^{-1}=\hat{a}_{\alpha_{\phi} f}$, we have

$$
\begin{aligned}
\hat{U}(\phi) \hat{a}_{\bar{f}} \hat{U}(\phi)^{-1} & =\hat{a}_{\alpha_{\phi} \bar{f}}=\hat{a} \overline{\alpha_{\phi} f}=\left(\hat{a}_{\alpha_{\phi} f}\right)^{\dagger} \\
& =\left(\hat{U}(\phi) \hat{a}_{f} \hat{U}(\phi)^{-1}\right)^{\dagger}=\left(\hat{U}(\phi)^{\dagger}\right)^{-1} \hat{a}_{f} \hat{U}(\phi)^{\dagger} .
\end{aligned}
$$

Multiplying with the respective operators $\hat{U}(\phi)^{\dagger}, \hat{U}(\phi)$ from the right and the left, one gets

$$
\hat{U}(\phi)^{\dagger} \hat{U}(\phi) \hat{a}_{f}=\hat{a}_{f} \hat{U}(\phi)^{\dagger} \hat{U}(\phi)
$$

So the operator $\hat{I}_{\phi}$ commutes with all elements $\hat{a}_{f}$, for $f \in C(\overline{\mathcal{A}})$. Let $\Omega$ be the cyclic and separating ${ }^{6}$ vector for $C(\overline{\mathcal{A}})$ (corresponding to the function $f(A) \equiv 1$, then, since $\Omega$ is also cyclic for the cylindrical functions $C y l$, there is a sequence of cylindrical functions $f_{n} \in C y l$ such that

$$
\lim _{n \rightarrow \infty} \hat{a}_{f_{n}} \Omega=\hat{I}_{\phi} \Omega
$$

in the topology of $\mathcal{H}_{\text {kin }}$. Thus, for any two continuous functions $g, h \in C(\overline{\mathcal{A}})$, one has

$$
\begin{aligned}
\left\langle g \mid \hat{I}_{\phi} h\right\rangle & =\left\langle g \mid \hat{I}_{\phi} \hat{a}_{h} \Omega\right\rangle=\left\langle g \mid \hat{a}_{h} \hat{I}_{\phi} \Omega\right\rangle \\
& =\lim _{n \rightarrow \infty}\left\langle g \mid \hat{a}_{h} \hat{a}_{f_{n}} \Omega\right\rangle=\lim _{n \rightarrow \infty}\left\langle g \mid \hat{a}_{f_{n}} h\right\rangle
\end{aligned}
$$

since all the $\hat{a}_{h}$ are continuous operators on $\mathcal{H}_{\text {kin }}$ and are mutually commuting. Since the $|g\rangle,|h\rangle$ are a dense subset of $\mathcal{H}_{\text {kin }}$, the statement follows.

Lemma (4.7) is in fact true for all invertible maps $\phi: \overline{\mathcal{A}} \rightarrow \overline{\mathcal{A}}$. That the corresponding sequence of $f_{n}$ converges to the constant function on $\overline{\mathcal{A}}$ however, is a consequence of Lemma 4.6.

Lemma 4.8 Let $\phi \in \mathcal{A} u t(\mathcal{P})$ and $f_{n}$ be a sequence in $C y l$ such that $\hat{a}_{f_{n}}$ converges weakly to $\hat{I}_{\phi}=\hat{U}(\phi)^{\dagger} \hat{U}(\phi)$. Then, for every edge e with $s(e) \neq t(e)$ there is an $n_{0} \in \mathbb{N}$ such that $f_{n}$ depend trivially on all edges which are not e for all $n>n_{0}$.

\footnotetext{
${ }^{6}$ For an introduction to the $C^{*}$-algebraic notions, see e.g. $[4,17]$
} 
Proof: Let $f$ be a nontrivial function cylindrical over $e$. Then by lemma 4.6

$$
\left\langle f \mid \hat{I}_{\phi} f\right\rangle=\langle\hat{U}(\phi) f \mid \hat{U}(\phi) f\rangle=\langle f \mid f\rangle=\|f\|^{2}>0
$$

it follows with lemma 4.7 that $\left\langle f \mid \hat{a}_{f_{n}} f\right\rangle>0$ for some $n>n_{0}$. But if $f_{n}$ would depend nontrivially on any edge different from $e$, then so would the product $f_{n} f$, but not $f$, so $\left\langle f \mid \hat{a}_{f_{n}} f\right\rangle$ would be zero. Hence, $f_{n}$ cannot depend on any edge not being $e$ for $n>n_{0}$.

Corollary 4.3 There is an $n_{0} \in \mathbb{N}$ such that $f_{n}$ is the constant function 1 on $\overline{\mathcal{A}}$ for every $n>n_{0}$.

Proof: Choose two different edges $e_{1}, e_{2}$ which have differing beginning- and endpoints. Then $f_{n}$ depend trivially on every edge not equal $e_{1}$ for $n>n_{1}$ and trivially on every edge not equal to $e_{2}$ for $n>n_{2}$. But since $e_{1} \neq e_{2}, f_{n}$ depend trivially on every edge for $n>n_{0}:=\max \left(n_{1}, n_{2}\right)$. But the only cylindrical functions depending trivially on every edge are the constant functions. But since $\hat{U}(\phi) \Omega=\Omega$, we have

$$
\left\langle\Omega \mid \hat{I}_{\phi} \Omega\right\rangle=\langle\Omega \mid \Omega\rangle=1
$$

so $\hat{a}_{f_{n}}$ must be the constant function 1 for all $n>n_{0}$.

We conclude this section by stating the main result.

Theorem 4.1 For any automorphism $\phi \in \mathcal{A} u t(\mathcal{P})$ the corresponding operator $\hat{U}(\phi)$ on $\mathcal{H}_{\text {kin }}$ is unitary.

Proof: From corollary 4.3 it follows that the $\hat{a}_{f_{n}}$ converge to the identity operator. But with lemma 4.7 we conclude that $\hat{I}_{\phi}$ must be the identity operator. So $\hat{U}(\phi)^{\dagger} \hat{U}(\phi)=\mathbb{1}$, and multiplying by $\hat{U}(\phi)^{-1}$ from the right gives the desired result.

\section{The automorphism-invariant Hilbert space}

We now review the definition of the $\operatorname{Diff}(\Sigma)$-invariant Hilbert space for the case $G=S U(2)$ and $\operatorname{Diff}(\Sigma)$ being the group of analytical diffeomorphisms on $\Sigma[1,7]$.

Since there is no known nontrivial topology or Borel measure on Diff $(\Sigma)$, the group of (analytical) diffeomorphisms, defining the rigging map naively via the group averaging:

$$
\eta[\psi](\chi)=\int_{\operatorname{Diff}(\Sigma)} d \mu(\phi)\langle\psi \mid \hat{U}(\phi) \chi\rangle
$$

is not possible. But there are other ways of defining a antilinear map

$$
\eta: D \longrightarrow D_{\text {Diff }}^{*}
$$

from a dense subspace $D \subset \mathcal{H}_{\text {kin }}$ invariant under $\operatorname{Diff}(\Sigma)$ into the linear functionals on $D$ that are invariant under $\operatorname{Diff}(\Sigma)$. This is usually done as follows: 
For any $\phi \in \operatorname{Diff}(\Sigma)$ and any graph $\gamma$, also $\phi(\gamma)$ is a graph. It follows that, in the case of $G=S U(2)$, which we consider here, spin network functions $T_{\gamma, \vec{j}, \vec{n}, \vec{m}}$ are mapped into spin network functions. So for two SNFs one has that $\left\langle T_{\gamma, \vec{j} \vec{n}, \vec{m}} \mid \hat{U}(\phi) T_{\gamma^{\prime}, \vec{j}^{\prime} \vec{n}^{\prime}, \vec{m}^{\prime}}\right\rangle$ is either 0 or 1 , depending on $\phi \in \operatorname{Diff}(\Sigma)$. Then $\eta$ is defined by

$$
\eta\left[T_{\gamma, \vec{j}, \vec{n} \vec{m}}\right]\left(T_{\gamma^{\prime}, \vec{j}^{\prime} \vec{n}^{\prime}, \vec{m}^{\prime}}\right):=\sum_{\varphi \in \operatorname{Diff}(\Sigma) / \operatorname{Diff}_{\gamma}} F\left(\left|G S_{\gamma}\right|\right) \sum_{\tilde{\varphi} \in G S_{\gamma}}\left\langle\hat{U}(\varphi \circ \tilde{\varphi}) T_{\gamma, \vec{j}, \vec{n} \vec{m}} \mid T_{\gamma^{\prime}, \vec{j}^{\prime} \vec{n}^{\prime}, \vec{m}^{\prime}}\right\rangle
$$

where Diff ${ }_{\gamma}$ is the set of diffeomorphisms which leave $\mathcal{P}_{\gamma}$ invariant. $G S_{\gamma}$ is the group of graph symmetries, i.e. the quotient of $\operatorname{Diff}_{\gamma}$ and the subgroup of $\operatorname{Diff}(\Sigma)$ which leaves $\gamma$ invariant. This is a finite group. $F$ is a function, which can be chosen arbitrarily. This defines an antilinear map from the span of the SNFs to the $\operatorname{Diff}(\Sigma)$-invariant linear functionals, which serves as rigging map, and defines a $\operatorname{Diff}(\Sigma)$-invariant inner product via

$$
\langle\eta[\psi] \mid \eta[\chi]\rangle_{\text {Diff }}:=\eta[\psi](\chi)
$$

In the case of $\operatorname{Diff}(\Sigma)$, an orthogonal basis for $\mathcal{H}_{\text {diff is }}$ given by the set of equivalence classes of SNFs under the action of $\operatorname{Diff}(\Sigma)$. The normalization of these vectors is a nontrivial issue [18], and is governed by the function $F$ in (5.3).

If we now replace the diffeomorphisms $\operatorname{Diff}(\Sigma)$ by the automorphisms $\mathcal{A} u t(\mathcal{P})$, we can try to use the same techniques to define an automorphism-invariant inner product $\langle\cdot \mid \cdot\rangle_{\text {Aut }}$ and an automorphism-invariant Hilbert space $\mathcal{H}_{\text {Aut }}$.

\subsection{Graph combinatorics}

The automorphisms $\mathcal{A} u t(\mathcal{P})$ act unitarily on the Hilbert space $\mathcal{H}_{\text {kin }}$, as was demonstrated in the last section. Furthermore, we have seen that there are many automorphisms $\phi \in \mathcal{A} u t(\mathcal{P})$ that do not correspond simply to a piecewise analytic map $\Sigma \rightarrow \Sigma$. In particular, given any bijection $b: \Sigma \rightarrow \Sigma$, any choice of paths $p_{x} \in \operatorname{Mor}(x, b(x))$ defines a functor $\phi_{b, p} \in \mathcal{N}$. On the other hand, each edgeinterchanger acts trivially on the objects in $\Sigma$, but changes morphisms. Thus the automorphisms allow for a lot of freedom how to change metagraphs $\mu \in \mathcal{M}$. In this section we will present a lemma that shows how large the orbit of a graph is under the action of $\mathcal{A} u t(\mathcal{P})$.

Lemma 5.1 Given any two hyphs $v_{1}=\left(p_{1}, \ldots, p_{H}\right)$ and $v_{2}=\left(q_{1}, \ldots, q_{H}\right)$. Assume the two are combinatorially the same, i.e. there is a bijection b between the vertices $b: V\left(v_{1}\right) \rightarrow V\left(v_{2}\right)$ and a bijection $c:\left\{p_{1}, \ldots, p_{H}\right\} \rightarrow\left\{q_{1}, \ldots, q_{H}\right\}$ such that

$$
s\left(c\left(p_{k}\right)\right)=b\left(s\left(p_{k}\right)\right), \quad t\left(c\left(p_{k}\right)\right)=b\left(t\left(p_{k}\right)\right) .
$$

Then there is an automorphism $\phi \in \mathcal{A} u t(\mathcal{P})$ such that $\phi\left(v_{1}\right)=v_{2} \cdot{ }^{7}$

\footnotetext{
${ }^{7}$ Note that this is slightly weaker than demanding that every automorphism between the subgroupoids $\mathcal{P}_{v_{1}} \rightarrow \mathcal{P}_{v_{2}}$ can be extended to an automorphism on $\mathcal{P}$.
} 
Proof: We will explicitly construct this automorphism as a product of finitely many natural transformations of the identity and edge-interchangers. First we show that there is a sequence of edge-interchangers such that $v_{1}$ can be mapped to a graph $\gamma$, which has the same vertices as $v_{1}$, as well as the same combinatorics as $v_{1}$.

Choose a graph $\gamma^{\prime}=\left\{e_{1}^{\prime}, \ldots, e_{E^{\prime}}^{\prime}\right\}$ such that $v_{1} \leq \gamma^{\prime}$. Then, by definition, there is an edge $e_{l}^{\prime}$ in $\gamma^{\prime}$ such that $e_{l}^{\prime}$ meets $\left\{p_{1}, \ldots, p_{H-1}\right\}$ at most in its endpoints.

$$
p_{H}=\tilde{p}_{1} \circ e_{l}^{\prime} \circ \tilde{p}_{2}
$$

Choose an analytic curve $c$ (i.e. [[[c]]] is an edge) from $s\left(p_{H}\right)$ to $t\left(p_{H}\right)$ that does not meet any of the $e_{k}^{\prime}$, apart from the points $s\left(p_{H}\right)$ and $t\left(p_{H}\right)$. Then choose two curves $c_{1}$ and $c_{2}$ with the following properties:

- $s\left(c_{1}\right)=c\left(\frac{1}{4}\right)$ and $t\left(c_{1}\right)=s\left(e_{l}^{\prime}\right)$

- $s\left(c_{2}\right)=c\left(\frac{3}{4}\right)$ and $t\left(c_{2}\right)=t\left(e_{l}^{\prime}\right)$

- The curves $c_{1}, c_{2}$ are injective and $r\left(c_{1}\right), r\left(c_{2}\right)$ do not intersect, as well as they do not intersect with any of the $r(c), r\left(e_{k}^{\prime}\right)$, apart from their beginning- and endpoints.

For any curve $c$ and $0 \leq t_{1}, t_{2} \leq 1$ denote by $c^{t_{1}, t_{2}}$ the curve

$$
c^{t_{1}, t_{2}}(t):=c\left(t_{1}+t\left(t_{2}-t_{1}\right)\right)
$$

Then by construction the following paths

$$
\begin{aligned}
& e_{1}:=\left[\left[\left[c_{1}^{\frac{1}{2}, 1}\right]\right]\right] \circ e_{l}^{\prime} \circ\left[\left[\left[c_{2}^{\frac{1}{2}, 1}\right]\right]\right]^{-1} \\
& e_{2}:=\left[\left[\left[c_{1}^{0, \frac{1}{2}}\right]\right]\right]^{-1} \circ\left[\left[\left[c^{\frac{1}{4}, \frac{3}{4}}\right]\right]\right] \circ\left[\left[\left[c_{2}^{0, \frac{1}{2}}\right]\right]\right]
\end{aligned}
$$

are paths without self-intersection that mutually intersect only at their beginningand endpoints. Furthermore, they intersect with the other edges $e_{k}^{\prime}, k \neq l$ only at most in $s\left(e_{l}^{\prime}\right), t\left(e_{l}^{\prime}\right)$. Thus, by construction, the same is true for the paths $p_{1}, \ldots, p_{H-1}$, since they are built from the $e_{k}^{\prime}, k \neq l$.

Now choose representative curves $d_{1}$ and $d_{2}$ for $e_{1}$ and $e_{2}$ such that

- $d_{1}\left(\frac{1}{4}\right)=s\left(e_{l}^{\prime}\right)$ and $d_{1}\left(\frac{3}{4}\right)=t\left(e_{l}^{\prime}\right)$

- $d_{2}\left(\frac{1}{4}\right)=c\left(\frac{1}{4}\right)$ and $d_{1}\left(\frac{3}{4}\right)=c\left(\frac{3}{4}\right)$.

Finally choose for every $t \in(0,1) \backslash\left\{\frac{1}{4}, \frac{3}{4}\right\}$ a path $p_{t}$ that goes from $d_{1}(t)$ to $d_{2}(t)$, and which does not meet $e_{1}, e_{2}$ apart from their respective beginning- and endpoint. Furthermore, define

$$
\begin{aligned}
& p_{\frac{1}{4}}:=\left(\tilde{p}_{1}\right)^{-1} \circ\left[\left[\left[c^{0, \frac{1}{4}}\right]\right]\right] \\
& \left.p_{\frac{3}{4}}:=\tilde{p}_{2} \circ\left[\left[\left[c^{\frac{3}{4}}, 1\right]\right]\right]\right]^{-1} .
\end{aligned}
$$

Now we have chosen two paths without self-intersections $e_{1}, e_{2}$ which meet only in their beginning- and endpoint, a parametrization $d_{1}, d_{2}$ for each of the paths, and for each $t \in(0,1)$ a path $p_{t} \in \operatorname{Mor}\left(d_{1}(t), d_{2}(t)\right)$ that does not meet $e_{1}, e_{2}$, apart 
from its beginning- and endpoint. This data defines an automorphism $\phi \in \mathcal{A} u t(\mathcal{P})$ by lemma 3.1 .

Since $e_{1}, e_{2}$ have been chosen to be such that they meet the $p_{1}, \ldots, p_{H-1}$ only in at most finitely many points, we conclude that $\phi\left(p_{k}\right)=p_{k}$ for $k=1, \ldots, H-1$. By the definition of $\phi$ and (5.8), (5.9), we have

$$
\begin{aligned}
\phi\left(p_{H}\right) & =\phi\left(\tilde{p}_{1}\right) \circ \phi\left(e_{l}^{\prime}\right) \circ \phi\left(\tilde{p}_{2}\right) \\
& =\tilde{p}_{1} \circ\left(p_{\frac{1}{4}} \circ\left[\left[\left[c^{\frac{1}{4}, \frac{3}{4}}\right]\right]\right] \circ p_{\frac{3}{4}}^{-1}\right) \circ \tilde{p}_{2} \\
& =\left[\left[\left[c^{0, \frac{1}{4}}\right]\right]\right] \circ\left[\left[\left[c^{\frac{1}{4}, \frac{3}{4}}\right]\right]\right] \circ\left[\left[\left[c^{\frac{3}{4}, 1}\right]\right]\right] \\
& =[[[c]]] .
\end{aligned}
$$

So by this construction, all paths $p_{k}$ have been left invariant, except for $p_{H}$ which has been mapped to an edge $e_{H}:=[[[c]]]$, which meets the $p_{k}, k<H$ at most in its beginning- and endpoint. For this construction it was crucial that $p_{H}$ had a free segment w.r.t all the other $p_{k}, k<H$, but also w.r.t. $e_{H}$, by construction. This means that $\left(e_{H}, p_{1}, \ldots, p_{H-1}\right)$ is also a hyph, i.e. each of the $p_{k}$ has a free segment w.r.t all $p_{l}, l<k$, but also w.r.t $e_{H}$. So the same construction can be carried out once again to obtain an automorphism $\phi^{\prime} \in \mathcal{A} u t(\mathcal{P})$ that leaves $e_{H}$ as well as all $p_{k}, k>H-1$ invariant, but maps $p_{H-1}$ to an edge $e_{H-1}$ which meets $e_{H}, p_{k}, k<H-1$ at most in its beginning-and endpoint. This gives a hyph $\left(e_{H-1}, e_{H}, p_{1}, \ldots, p_{H-2}\right)$. By repeating this process, we arrive at a hyph $\left(e_{1}, \ldots, e_{H}\right)$, where all the $e_{k}$ are edges and meet at most in their beginning- and endpoint. So $\gamma:=\left\{e_{1}, \ldots, e_{H}\right\}$ is a graph. Thus, we have constructed a finite sequence of automorphisms that map the hyph $\left(p_{1}, \ldots, p_{H}\right)$ into the graph $\gamma$.

Now let $\gamma^{\prime \prime}=\left\{e_{1}^{\prime \prime}, \ldots, e_{H}^{\prime \prime}\right\}$ be any graph which has the same combinatorics as $\gamma=\left\{e_{1}, \ldots, e_{H}\right\}$, but the edges of which do not intersect with the edges of $\gamma$. $V(\gamma)=\left\{v_{1}, \ldots, v_{V}\right\}$ and $V\left(\gamma^{\prime \prime}\right)=\left\{v_{1}^{\prime \prime}, \ldots, v_{V}^{\prime \prime}\right\}$, and

$$
s\left(e_{k}\right)=v_{a}, t\left(e_{k}\right)=v_{b} \quad \Leftrightarrow \quad s\left(e_{k}^{\prime \prime}\right)=v_{a}^{\prime \prime}, t\left(e_{k}^{\prime \prime}\right)=v_{b}^{\prime \prime} .
$$

Then construct an automorphism mapping one to the other by the following method: For each $v_{l} \in V(\gamma)$ choose a path $p_{v_{l}} \in \operatorname{Mor}\left(v_{l}, v_{l}^{\prime \prime}\right)$ such that all $p_{v_{l}}$ are without self-intersections, do not intersect each other, and intersect the $e_{k}, e_{k}^{\prime \prime}$ only in the vertices $v_{l}, v_{l}^{\prime \prime}$. Then define a natural transformation of the identity $\phi_{b, p} \in \mathcal{N}$ by the following data:

$$
\begin{aligned}
b\left(v_{l}\right):=v_{l}^{\prime \prime}, \quad b\left(v^{\prime \prime}\right):=v_{l}, \quad b(x)=x \text { else } \\
p_{v_{l}}:=p_{v_{l}}, \quad p_{v_{l}^{\prime \prime}}:=p_{v_{l}}^{-1}, \quad p_{x}=\mathrm{id}_{x} \text { else }
\end{aligned}
$$

Then each of the $p_{k}^{\prime}:=\phi_{b, p}\left(e_{k}\right)$ is a path without self-intersections, and all the $p_{k}^{\prime}$ have free segments w.r.t each other (in particular the $e_{k}$ ). So $\left(p_{1}^{\prime}, \ldots, p_{H}^{\prime}\right.$ ) is a hyph with vertices $v_{1}^{\prime \prime}, \ldots, v_{V}^{\prime \prime}$. On the other hand, $\gamma^{\prime \prime}$ is a graph, the edges $e_{k}^{\prime \prime}$ of which intersect all the $p_{k}^{\prime}$ at most in the vertices $v_{l}^{\prime \prime}$. Since for each path $p_{k}^{\prime}$, the edge $e_{k}^{\prime}$ starts and ends at the same points as $p_{k}^{\prime}$ by construction, there is a sequence of $H$ 
edge-interchangers that maps each $p_{k}^{\prime}$ to $e_{k}^{\prime \prime}$.

So we have started with a hyph $v_{1}=\left(p_{1}, \ldots, p_{H}\right)$, have mapped $v_{1}$ to $\gamma=$ $\left(e_{1}, \ldots, e_{H}\right)$ by a sequence of $H$ edge-interchangers, have mapped $\gamma$ by a natural transformation of the identity $\phi_{b, p}$ to the hyph $v_{1}^{\prime}=\left(p_{1}^{\prime}, \ldots, p_{H}^{\prime}\right)$, and have mapped $v_{1}^{\prime}$ by another sequence of $H$ edge-interchangers to any other graph $\gamma^{\prime \prime}$, of which we only demanded that it has the same combinatorics as $v_{1}$, and that it has empty intersection with $\gamma$. By the same construction, we can show that we can map any two graphs that have the same combinatorics but empty intersection into each other by a sequence of automorphisms. But this shows that one can map any two hyphs $v_{1}, v_{2}$ having the same combinatorics into each other by a sequence of edge-interchangers and natural transformations of the identity. Thus, the lemma is proven.

\subsection{Orbits of the Automorphisms}

In order to define the automorphism-invariant Hilbert space $\mathcal{H}_{\text {Aut }}$, it is instructive to get an idea of the orbits of vectors $\psi \in \mathcal{H}_{\text {kin }}$ under the action of $\mathcal{A} u t(\mathcal{P})$. First we will investigate these orbits in the following for arbitrary gauge groups $G$, in order to arrive at some general statements. Then, we will specialize to $G=U(1)$ and $G=S U(2)$ in order to say something about the corresponding spaces $\mathcal{H}_{\text {Aut }}$.

Lemma 5.2 Let $f \in C y l$ be a gauge-invariant cylindrical function. Then for any natural transformation of the identity $\phi_{b, p} \in \mathcal{N}$ one has

$$
\hat{U}\left(\phi_{b, p}\right) f=f
$$

Proof: Let $f$ be cylindrical over the graph $\gamma=\left\{e_{1}, \ldots, e_{E}\right\}$. For each $v \in V(\gamma)$ and each $a \in \overline{\mathcal{A}}$ denote $g_{v}^{A}:=A\left(p_{v}\right) \in G$. With

$$
f(A)=F\left(A\left(e_{1}\right), \ldots, A\left(e_{E}\right)\right)
$$

we get

$$
\begin{aligned}
\left(\hat{U}\left(\phi_{b, p}\right) f\right)(A) & =f\left(\alpha_{\phi_{b, p}} A\right) \\
& =F\left(A\left(p_{s\left(e_{1}\right)}^{-1} \circ e_{1} \circ p_{t\left(e_{1}\right)}\right), \ldots, A\left(p_{s\left(e_{E}\right)}^{-1} \circ e_{E} \circ p_{t\left(e_{E}\right)}\right)\right) \\
& =F\left(\left(g_{s\left(e_{1}\right)}^{A}\right)^{-1} A\left(e_{1}\right) g_{t\left(e_{1}\right)}, \ldots,\left(g_{s\left(e_{E}\right)}^{A}\right){ }^{-1} A\left(e_{E}\right) g_{t\left(e_{E}\right)}\right) \\
& =F\left(A\left(e_{1}\right), \ldots, A\left(e_{E}\right)\right) \\
& =f(A) .
\end{aligned}
$$

Lemma 5.2 shows one important fact: Although $\phi_{b, p}$ can change graphs quite arbitrarily, the corresponding functions on that graph remain unchanged. The reason for this is that a function cylindrically over a metagraph $\mu \in \mathcal{M}$ does generally not carry all of the information in order to reconstruct $\mu$ from its dependence $A \rightarrow f(A)$.

Consider the following example: Given a metagraph $\mu=\left\{p_{1}, \ldots, p_{4}\right\}$, as in picture 5.2. 


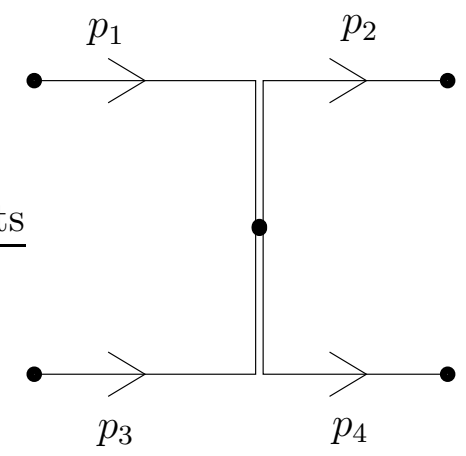

Figure 5.1: A metagraph $\mu$ (in fact a hyph) consisting of four paths and five vertices.

Note that the paths $p_{1} \circ p_{2}$ and $p_{3} \circ p_{4}$ each contain a retracing. Now consider the function cylindrical over the metagraph $\mu$ by

$$
f(A)=F\left(A\left(p_{1}\right), A\left(p_{2}\right), A\left(p_{3}\right), A\left(p_{4}\right)\right)
$$

with $F\left(h_{1}, h_{2}, h_{3}, h_{4}\right)=\tilde{F}\left(h_{1} h_{2}, h_{3} h_{4}\right)$ for some smooth function $\tilde{F}$ on $G^{2}$. Note that $f$ does not depend on the parallel transports along all the $p_{1}, \ldots, p_{4}$, but only on the ones along $p_{1} \circ p_{2}$ and $p_{3} \circ p_{4}$. So in particular, $f$ does not depend at all on the parallel transports along the retracings. Consequently, $f$ is cylindrical over the graph $\gamma$, which consists of the following two edges:

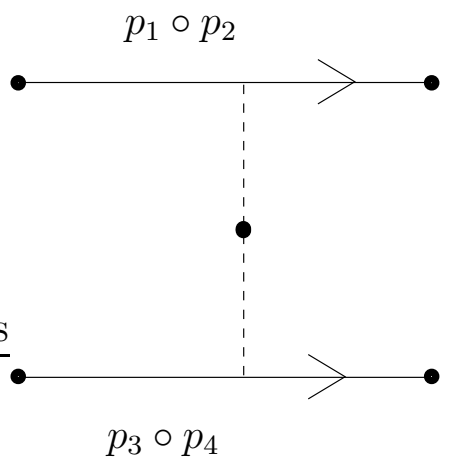

Figure 5.2: The function $f$ given by (5.16) is also cylindrical over the graph $\tilde{\gamma} \leq \mu$, which only consists of two edges and four vertices

So we see that the dependence of the function $f$ is not on all of $\mu$, but only of a certain subgraph. This is of particular importance, since the following graph $\gamma$ :

has the same combinatorics as $\mu$. So, by lemma 5.1 there is an automorphism $\phi \in \mathcal{A} u t(\mathcal{P})$ mapping one to the other, i.e. $\phi\left(e_{k}\right)=p_{k}$. Thus, a function $f$ cylindrical over $\gamma$ is mapped into $\alpha_{\phi} f$, which is cylindrical over $\mu$. But if $f$ depends only on the parallel transports along $e_{1} \circ e_{2}$ and $e_{3} \circ e_{4}$, then $\alpha_{\phi}$ is also cylindrical over $\tilde{\gamma} \leq \mu$, as we have just seen. So, although $\phi$ respects the number of vertices and paths in a metagraph, $\hat{U}(\phi)$ can map a function which is cylindrical over a metagraph 


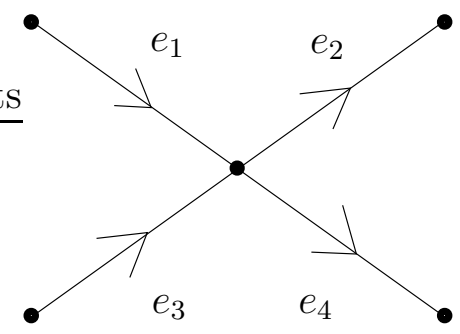

Figure 5.3: The metagraph $\mu$ can be mapped to this graph $\gamma$ by an automorphism

to a function cylindrical over another metagraph, which does not have the same combinatorics, such as $\gamma$ and $\tilde{\gamma}$ in the example above.

Although this seem paradoxical at first, it is in fact quite natural: Consider the function $f$, which is cylindrical over the graph $\gamma$, as in figure (5.2), and which depends only on the parallel transports along $e_{1} \circ e_{2}$ and $e_{3} \circ e_{3}$. Then the function does not "know" that it is cylindrical over a graph with four edges, in particular it does not know anything about the middle vertex $t\left(e_{1}\right)$. For instance, it is automatically gauge-invariant w.r.t. gauging at this vertex. This does, of course, not happen to an arbitrary function $g$ cylindrical over $\gamma$, whose dependence on the parallel transports along all four edges $e_{1}, \ldots, e_{4}$ is nontrivial.

We see that the cylindrical functions can carry much less information than the graph that they are cylindrical over. In particular, the functions only carry information about on how many parallel transports they depend, and which of these start or end at the same points. It is exactly this information that is preserved by the automorphisms.

This is of particular importance for the gauge-invariant functions, since these carry only information about the first fundamental class of the graph, but not about the graph topology itself, which is summarized by the following lemma.

Lemma 5.3 Let $f \in C y l$ be a gauge-invariant cylindrical function over a graph $\gamma$ with $E$ edges and $V$ vertices. Then there is a $E-V+1$-flower graph $\tilde{\gamma}$ (a graph with one vertex and $E-V+1$ edges all starting and ending at that vertex) and an automorphism $\phi \in \mathcal{A} u t(\mathcal{P})$ such that $\hat{U}(\phi) f$ is a gauge-invariant function cylindrical over $\tilde{\gamma}$.

Proof: Choose a maximal tree $\tau$ in the graph $\gamma$. Choose a vertex $x \in V(\tau)$ and for each edge $e_{l} \in E(\gamma) \backslash E(\tau)$ not belonging to the tree denote the unique path from $x$ to $s\left(e_{l}\right)$ lying in $\tau$ as $p_{l}^{1}$, and from $v$ to $t\left(e_{l}\right)$ as $p_{l}^{2}$. Then define the $E-V+1$ paths

$$
p_{l}:=p_{l}^{1} \circ e_{l} \circ\left(p_{l}^{2}\right)^{-1} \text {. }
$$

Then $v=\left(p_{1}, \ldots, p_{E-V+1}\right)$ is a hyph, since each path $p_{l}$ contains $e_{l}$, which is a free segment for all the other paths. Note that, due to gauge-invariance, the function $f$ only depends on the parallel transports along the paths $p_{l}$ (see, e.g. [19]). In particular, $f$ is cylindrical over the hyph $v:=\left(p_{1}, \ldots, p_{E-V+1}\right): f \in C y l(v)$. But since all paths $p_{l}$ in $v$ start and end in the vertex $x$, by lemma 5.1 there is an automorphism mapping the hyph $v$ to an $E-V+1$-flower graph $\tilde{\gamma}$. So $f$ gets mapped 


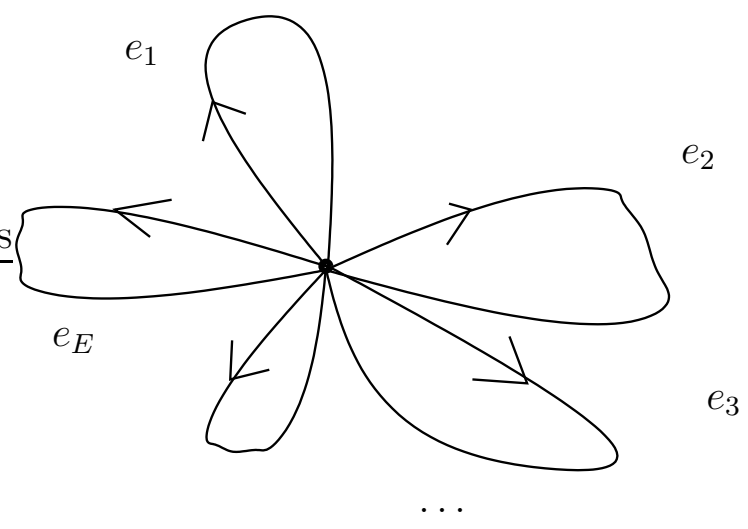

Figure 5.4: An E-flower graph.

to a function $\alpha_{\phi} f$ being cylindrical over that flower graph.

Lemma 5.3 shows the tremendous size that the orbits of a vector under the action of $\mathcal{A} u t(\mathcal{P})$ have. In the case of the abelian gauge group $G=U(1)$, the size is so large that one can in fact compute the orbits, in the sense that one can determine the set of equivalence classes of vectors in $\mathcal{H}_{\text {kin }}$ under the action of $\mathcal{A} u t(\mathcal{P})$.

\subsection{The automorphism-invariant Hilbert space for gauge group $G=U(1)$}

Cylindrical functions for gauge group $G=U(1)$ carry particularly few information about the graph they are cylindrical over.

Lemma 5.4 Let $\gamma=\left\{e_{1}, \ldots, e_{E}\right\}$ be an E-flower graph. Consider a word $\vartheta\left(e_{2}, \ldots, e_{E}\right)$ in the edges of $\gamma$, apart from $e_{1}$. Since $\gamma$ is a flower graph, $\vartheta$ is a path in the groupoid $\mathcal{P}_{\gamma}$, i.e. a path starting and ending at the one vertex in $V(\gamma)$, and going through the edges $e_{2}, \ldots, e_{E}$ in an order determined by the word $\vartheta$. Then there is an automorphism $\phi \in \mathcal{A} u t(\mathcal{P})$ with the following properties:

$$
\begin{aligned}
\phi: e_{1} & \longmapsto e_{1} \circ \vartheta\left(e_{2}, \ldots, e_{E}\right) \\
e_{k} & \longmapsto e_{k}, \quad \text { for } k \in\{2, \ldots, E\}
\end{aligned}
$$

Proof: Note that $\left(e_{2}, \ldots, e_{E}, e_{1} \circ \vartheta\right)$ is a hyph, since $e_{1} \circ \vartheta\left(e_{2}, \ldots, e_{E}\right)$ has a free segment w.r.t. $e_{2}, \ldots, e_{E}$ (which is $\left.e_{1}\right)$, and the remaining $\left(e_{2}, \ldots, e_{E}\right)$ form a graph. Since $\left(e_{1}, \ldots, e_{E}\right)$ is also a hyph, having the same combinatorics, the assertion follows directly from lemma 5.1.

Lemma 5.5 Let $\gamma$ be an E-flower graph, $\vec{n} \in \mathbb{Z}^{E}$ and $T_{\gamma, \vec{n}}$ be a charge network. Let $\left(m_{2}, \ldots, m_{E}\right) \in \mathbb{Z}^{E-1}$, then there is an automorphism $\phi \in \mathcal{A} u t(\mathcal{P})$ such that

$$
\hat{U}(\phi) T_{\gamma, \vec{n}}=T_{\gamma, \vec{n}^{\prime}}
$$


with

$$
\begin{aligned}
& n_{1}^{\prime}=n_{1} \\
& n_{k}^{\prime}=n_{k}+n_{1} m_{k} \quad \text { for } k \in\{2, \ldots, E\}
\end{aligned}
$$

Proof: Choose the word

$$
\vartheta\left(e_{2}, \ldots, e_{E}\right)=e_{2}^{m_{2}} \circ \ldots \circ e_{E}^{m_{E}} .
$$

Then, by lemma 5.4 , there is an automorphism $\phi \in \mathcal{A} u t(\mathcal{P})$ with

$$
\phi\left(e_{1}\right)=e_{1} \circ e_{2}^{m_{2}} \circ \ldots \circ e_{E}^{m_{E}},
$$

and which leaves all other $e_{k}$ invariant. Thus we get

$$
\begin{aligned}
\left(\alpha_{\phi} T_{\gamma, \vec{n}}\right)(A) & =\prod_{k=1}^{E} A\left(\alpha_{\phi} e_{k}\right)^{n_{k}} \\
& =\left[A\left(e_{1}\right) \prod_{k=2}^{E} A\left(e_{k}\right)^{m_{k}}\right]^{n_{1}} \prod_{k=2}^{E} A\left(e_{k}\right)^{n_{k}} \\
& =A\left(e_{1}\right)^{n_{1}} \prod_{k=2}^{E} A\left(e_{k}\right)^{n_{k}+n_{1} m_{k}} \\
& =T_{\gamma, \vec{n}^{\prime}}(A),
\end{aligned}
$$

where $T_{\gamma, \vec{n}^{\prime}}$ is given by $(5.20)$

Lemma 5.6 Let $\gamma$ be an E-flower graph, $\vec{n} \in \mathbb{Z}^{E}$, and $T_{\gamma, \vec{n}}$ a charge-network function. Then there is an automorphism $\phi \in \mathcal{A} u t(\mathcal{P})$ such that

$$
\alpha_{\phi} T_{\gamma, \vec{n}}=T_{\gamma,(p, 0,0, \ldots, 0)},
$$

where $p \in \mathbb{Z}$ is the greatest common divisor of the $\left|n_{1}\right|, \ldots,\left|n_{E}\right|$.

Proof: First assume that all $n_{k} \geq 0$. If this is not the case, one can, by lemma 5.1, find an automorphism that reverses the direction of all edges $e_{k}$ such that $n_{k}<0$ :

$$
\begin{array}{ll}
\phi\left(e_{k}\right)=e_{k}^{-1} & \text { if } n_{k}<0 \\
\phi\left(e_{k}\right)=e_{k} & \text { else }
\end{array}
$$

We will consider a sequence of 'steps' (5.20), each of which changes the charge distribution among the edges $e_{1}, \ldots, e_{E}$, and corresponds to the action of an automorphism $\phi \in \mathcal{A} u t(\mathcal{P})$. We will construct the sequence such that, at the end, the charge distribution $\left(n_{1}, \ldots, n_{E}\right)$ will have changed into $(p, 0,0, \ldots, 0)$.

First, assume that not all of the $n_{k}$ are zero, otherwise $T_{\gamma, \vec{n}}=\Omega$ is the vector corresponding to the constant function $f(A) \equiv 1$, and we are done. 
Choose one of the smallest $n_{k}$, which is without loss of generality $n_{1}$. Then choose another $n_{l}, l \geq 1$ with $n_{1} \leq n_{l}$. Then, define $m_{l}:=-1, m_{k}=0$ for $1 \neq k \neq l$. Then, by lemma 5.5 there is an automorphism $\phi^{(1)} \in \mathcal{A} u t(\mathcal{P})$ that maps $T_{\gamma, \vec{n}}$ to $T_{\gamma, \vec{n}^{(1)}}$ with $n_{k}^{(1)}=n_{k}$, for $k \neq l$, and $n_{l}^{(1)}=n_{l}-n_{k} \geq 0$. Continue with choosing one of the smallest $n_{k}^{(1)}$ (by renumbering, this is again wlog $n_{1}^{(1)}$ ), and choose one $n_{l}^{(1)}$ with $n_{1}^{(1)} \leq n_{l}^{(1)}$, define $m_{l}^{(1)}=-1, m_{k}^{(1)}=0$ for $1 \neq k \neq l$. By lemma 5.5 find an automorphism $\phi^{(2)}$ mapping $T_{\gamma, \vec{n}^{(2)}}$ with $n_{k}^{(2)}=n_{k}^{(1)}$ for $k \neq l$ and $n_{l}^{(2)}=n_{l}^{(1)}-n_{1}^{(1)} \geq 0$, choose the smallest nonzero $n_{k}^{(3)}$, and continue this algorithm.

Since each of the $\vec{n}^{(r)}$ is a sequence of nonnegative numbers, and in each step one of the numbers is reduced by some $n_{1}^{(r-1)}>0$, the algorithm stops after finitely many steps, say after $N \leq \sum_{k} n_{k}$ steps. The algorithm cannot be continued further, if after choosing the smallest $n_{1}^{(N)}$, there is no other nonzero $n_{l}^{(N)}$, i.e. if all other $n_{k}^{(N)}$ are zero. Then, we have constructed a series of automorphisms mapping $T_{\gamma, \vec{n}}$ to some $T_{\gamma,\left(n_{1}^{(N)}, 0,0, \ldots, 0\right)}$ :

$$
\hat{U}\left(\phi^{(N)}\right) \hat{U}\left(\phi^{(N-1)}\right) \cdots \hat{U}\left(\phi^{(1)}\right) T_{\gamma, \vec{n}}=T_{\gamma,(p, 0,0, \ldots, 0)}
$$

with $p=n_{1}^{(N)}$. It is easy to see that in each step, the greatest common divisor of the nonzero $n_{k}^{(r)}$ never changes. Thus, since $p$ is its own greatest common divisor, it has to be equal to the greatest common divisor of all the nonzero $n_{k}$. This completes the proof.

Lemma 5.7 Let $\gamma$ be a one-flower graph. If for $n, m \in \mathbb{Z}$ there is an automorphism $\phi \in \mathcal{A u t}(\mathcal{P})$ such that $\hat{U}(\phi) T_{\gamma, n}=T_{\gamma, m}$, then $n= \pm m$.

Assume there is a $\phi \in \mathcal{A} u t(\mathcal{P})$ such that $\hat{U}(\phi) T_{\gamma, n}=T_{\gamma, m}$. Then $\phi(\gamma)$ is a metagraph $\phi(\gamma) \leq \gamma$. Thus, $\phi(\gamma) \in \mathcal{P}_{\gamma}$. But $\mathcal{P}_{\gamma}=\left\{\gamma^{k} \mid k \in \mathbb{Z}\right\}$. Thus, $\phi(\gamma)=\gamma^{k}$ for some $k \in \mathbb{Z}$. Since $\hat{U}\left(\phi^{-1}\right) T_{\gamma, m}=T_{\gamma, n}$, we also have $\phi^{-1}(\gamma) \leq \gamma$, i.e. $\phi^{-1}(\gamma)=\gamma^{l}$ for some $l \in \mathbb{Z}$. On the other hand,

$$
\gamma=\phi\left(\phi^{-1}(\gamma)\right)=\phi\left(\gamma^{l}\right)=(\phi(\gamma))^{l}=\gamma^{k l}
$$

by the functorial properties of $\phi$. Thus, $k l=1$, but the only two pairs of integer numbers $k, l \in \mathbb{Z}$ with this property are $k=l=1$ and $k=l=-1$. So either $\phi(\gamma)=\gamma$ or $\phi(\gamma)=\gamma^{-1}$. The claim follows.

By lemma 5.3, every gauge-invariant charge-network function can be mapped by an automorphism to one on a flower graph (on which all charge-networks are automatically gauge-invariant). In lemma 5.6 we have seen that for gauge group $G=U(1)$, each charge-network function on a flower graph can be mapped by an automorphism into $T_{\gamma, n}$ for some one-flower graph $\gamma$ and some $n \in \mathbb{Z}$. This $n$ is unique up to a sign, as we have seen in Lemma 5.7. We have thus determined the complete set of orbits of charge-network states under the action of $\mathcal{A} u t(\mathcal{P})$. 
Lemma 5.8 Let $D$ be the linear span of all charge-network states in $\mathcal{H}_{\mathrm{kin}}$. Then the following map:

$$
\begin{aligned}
\eta & : D \longrightarrow D_{\mathcal{A} u t(\mathcal{P})}^{*} \\
\eta[f] g & :=\sum_{[\phi] \in \mathcal{A} u t(\mathcal{P}) / \sim}\langle f \mid \hat{U}(\phi) g\rangle
\end{aligned}
$$

defines an antilinear map from $D$ to the automorphism-invariant linear functionals over D. Here $\phi_{1} \sim \phi_{2}$ if $\left\langle f \mid \hat{U}\left(\phi_{1}\right) g\right\rangle=\left\langle f \mid \hat{U}\left(\phi_{2}\right) g\right\rangle$.

Proof: We have seen that for each charge-network state $T_{\gamma, \vec{n}}$ there is a unique $n \geq 0$ such that

$$
\left[T_{\gamma, \vec{n}}\right]=\left[\bigcirc_{n}\right]
$$

where $\bigcirc$ is a one-flower graph, $\bigcirc_{n}$ denotes the charge-network function on $\bigcirc$ given by the one charge $n$, and [.] denotes the orbit of - under $\mathcal{A} u t(\mathcal{P})$. Denote this $n$ by $\omega(\vec{n})$, then we have

$$
\sum_{[\phi] \in \mathcal{A} u t(\mathcal{P}) / \sim}\left\langle T_{\gamma, \vec{n}} \mid \hat{U}(\phi) T_{\gamma^{\prime}, \vec{n}^{\prime}}\right\rangle=\delta_{\omega(\vec{n}), \omega\left(\vec{n}^{\prime}\right)},
$$

due to the fact that any $\phi \in \mathcal{A} u t(\mathcal{P})$ maps a charge network into another charge network, so $\left\langle T_{\gamma, \vec{n}} \mid \hat{U}(\phi) T_{\gamma^{\prime}, \vec{n}^{\prime}}\right\rangle$ is either 0 or 1 .

With this rigging map, an inner product $\langle\cdot \mid \cdot\rangle_{\text {Aut }}$ can be defined on the set of all finite linear combinations of charge-network functions, and we thus see

Corollary 5.1 The automorphism-invariant inner product on $\eta(D)$ given by the rigging map (5.27) can be completed to the automorphism-invariant Hilbert space

$$
\mathcal{H}_{\mathrm{Aut}}=\left\{\left.\sum_{k=0}^{\infty} c_{n}\left[\bigcirc_{n}\right]\left|\sum_{n}\right| c_{n}\right|^{2}<\infty\right\}
$$

Proof: This is clear from the fact that the orbit of every finite linear combination $f$ of charge networks is given by

$$
[f]=\sum_{k=0}^{N} c_{n}\left[\bigcirc_{n}\right]
$$

and

$$
\left\langle\left[\bigcirc_{n}\right] \mid\left[\bigcirc_{m}\right]\right\rangle_{\mathrm{Aut}}=\delta_{n m}
$$

As we have seen, the automorphism-invariant Hilbert space can be computed directly, for a certain choice of rigging map (5.27). For each charge-network function $T_{\gamma, \vec{n}}$ on a graph $\gamma$, there is a natural number $n$ and an automorphism $\phi$ such that $\alpha_{\phi} T_{\gamma, \vec{n}}=\bigcirc_{n}$. This shows how tremendously large the orbits of the automorphism 
group are in the case of $G=U(1)$. The reason for this is the following: given any graph $\gamma$ with $E$ edges and a charge-network function $T_{\gamma, \vec{n}}$, then

$$
T_{\gamma, \vec{n}}(A)=e^{i\left(n_{1} \varphi_{1}+\cdots+n_{E} \varphi_{E}\right)}
$$

where $e^{i \varphi_{k}}=A\left(e_{k}\right)$ is the holonomy of $A$ along the edge $e_{k}$. Then, our previous results show that there is a closed loop $l$ in $\gamma$, i.e. a path consisting of edges in $\gamma$ and their inverses, which starts and ends at the same point, such that each $e_{k}$ is traversed exactly $n_{k}$ times (counting going against the orientation of $e_{k}$ as -1 ). Then

$$
T_{\gamma, \vec{n}}(A)=e^{i \varphi}
$$

with $\varphi=n_{1} \varphi_{1}+\cdots+n_{E} \varphi_{E}$, and $e^{i \varphi}=A(l)$ is the holonomy along $l$. So the charge-network function $T_{\gamma, \vec{n}}$ is cylindrical over the metagraph $l$. If $l=\tilde{l}^{n}$ for a simple loop $\tilde{l}$, then $T_{\gamma, \vec{n}}$ is also cylindrical over $\tilde{l}$, and we have shown that there is an automorphism $\phi$ mapping the loop $\tilde{l}$ to a (say) circle $\bigcirc$, hence $T_{\gamma, \vec{n}}$ to $\bigcirc_{n}$.

The above consideration rests crucially on the abelianess of $U(1)$. In particular, it does not matter in which order $l$ transverses the paths in $\gamma$, just how many times. This will not be true for non-abelian gauge groups, such as $G=S U(2)$, as we will see in the following.

\subsection{The automorphism-invariant Hilbert space for gauge group $G=S U(2)$.}

In this section, we investigate the set of orbits of vectors in $\mathcal{H}_{\text {kin }}$ under the action of $\mathcal{A} u t(\mathcal{P})$, in the case of $G=S U(2)$. Ultimately, the goal is to compute the set of linear functionals invariant under $\mathcal{A} u t(\mathcal{P})$ with some inner product, such as in the case for $G=S U(2)$, for example

$$
\begin{gathered}
\eta: D \longrightarrow D_{\mathcal{A} u t(\mathcal{P})}^{*} \\
\eta[f] g:=\sum_{[\phi] \in \mathcal{A} u t(\mathcal{P}) / \sim}\langle f \mid \hat{U}(\phi) g\rangle
\end{gathered}
$$

Again, $\phi_{1} \sim \phi_{2}$ if $\left\langle f \mid \hat{U}\left(\phi_{1}\right) g\right\rangle=\left\langle f \mid \hat{U}\left(\phi_{2}\right) g\right\rangle$.

To compute the set of orbits of vectors in $\mathcal{H}_{\text {kin }}$ under the action of $\mathcal{A} u t(\mathcal{P})$ for the case of $G=S U(2)$ is more difficult, due to the fact that for a spin network func-

tion $T_{\gamma, \vec{j}, \vec{n}, \vec{m}}$, the transformed $\alpha_{\phi} T_{\gamma, \vec{j}, \vec{n}, \vec{m}}$ is not necessarily a spin network function anymore. The same holds for any gauge-invariant spin network function $T_{\gamma, \vec{j}, \vec{I}}$ : If one chooses, for each graph $\gamma$, an orthonormal basis of intertwiners $\vec{I}$ at each vertex, then any such basis vector can be mapped to a finite linear combination of basis vectors by an automorphism 
From this difficulty arises the phenomenon that, for two vectors $T_{\gamma, \vec{I}}, T_{\gamma^{\prime}, \vec{j}^{\prime}, \vec{I}^{\prime}}$ the set of all possible overlaps

$$
\left\{\left\langle T_{\gamma, \vec{I}} \mid \alpha_{\phi} T_{\gamma^{\prime}, \vec{j}^{\prime}, \vec{I}^{\prime}}\right\rangle \mid \phi \in \mathcal{A} u t(\mathcal{P})\right\}
$$

is not just $\{0,1\}$, as in the case for piecewise analytic diffeomorphisms, or for automorphisms and gauge group $G=U(1)$. We consider a simple example:

We have already seen in lemma 5.3 that every gauge-invariant function on a graph can be mapped by an automorphism to a gauge-invariant function on a flower graph. Thus, it is sufficient to consider the orbits of gauge-invariant functions on flower graphs. Consider the ${ }^{8}$ 2-flower graph $\gamma=\left\{e_{1}, e_{2}\right\}$.

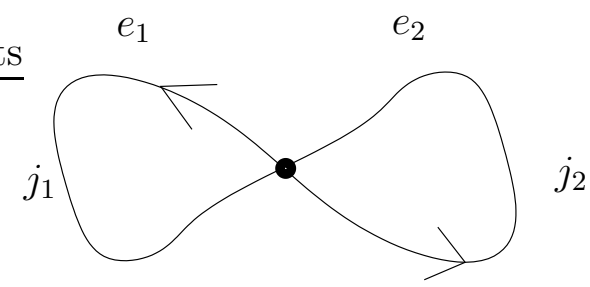

Figure 5.5: The 2-flower graph

For fixed $j_{1}, j_{2} \in \frac{1}{2} \mathbb{N}$, the intertwiner space is $j_{1}+j_{2}-\left|j_{1}-j_{2}\right|+1$-dimensional. Assume $j_{1}=j_{2}=\frac{1}{2}$. Then the intertwiner space is two-dimensional. If we choose the normalized vector

$$
T_{1}(A):=\operatorname{tr}_{\frac{1}{2}}\left(A\left(e_{1}\right)\right) \operatorname{tr}_{\frac{1}{2}}\left(A\left(e_{2}\right)\right)
$$

to be one of the two orthonormal basis vectors in the intertwiner space, and choose some normalized $T_{2}$ orthogonal to it, we get an orthonormal base of the intertwiner space. On the other hand, consider the one-flower graph (in fact, a Wilson loop) $\bigcirc=\{e\}$ having only one edge $e$, and the gauge-invariant function $T_{0}$ cylindric on given by

$$
T_{0}(A):=\operatorname{tr}_{\frac{1}{2}}(A(e)) .
$$

With the spin $j=\frac{1}{2}$ on the edge $e$, there is only one gauge-invariant function on $\bigcirc$ (the intertwiner-space is one-dimensional), which is given exactly by $T_{0}$. By lemma 5.1 however, we know that there is an automorphism $\phi$ such that $\phi(e)=e_{1} \circ e_{2}$, so $\alpha_{\phi} T_{0}$ is cylindrical over the two-flower graph $\gamma$, and in fact can be decomposed into $T_{1}, T_{2}$. Since

$$
\alpha_{\phi} T_{0}(A)=\operatorname{tr}_{\frac{1}{2}}\left(A\left(e_{1}\right) \cdot A\left(e_{2}\right)\right),
$$

\footnotetext{
${ }^{8}$ Technically, there are many flower graphs, but all of them can be mapped into each other by the automorphisms by Lemma 5.1, so in the following we speak of "the" 2-flower graph.
} 
a short calculation reveals that

$$
\left\langle T_{1} \mid \alpha_{\phi} T_{0}\right\rangle=\frac{1}{2}
$$

So

$$
\alpha_{\phi} T_{0}=\cos \frac{\pi}{3} T_{1}+\sin \frac{\pi}{3} T_{2}
$$

We see that, since there are nontrivial ways to embed an $E$-flower into an $E^{\prime}$ flower (with $E<E^{\prime}$ ) by "wrapping loops", certain basis vectors on the $E$-flower will be mapped to nontrivial combinations of basis vectors on the $E^{\prime}$-flower. All these nontrivial overlaps will show up in the automorphism-invariant inner product (5.35). To compute all the contributions is now a combinatorial task. We refrain from doing so here, but simply state a conjecture about the nature of the set of orbits of vectors in $\mathcal{H}_{\text {kin }}$ under the action of $\mathcal{A} u t(\mathcal{P})$.

In our example above, we have seen that the function $T_{1}$ and $\alpha_{\phi} T_{0}$ constitute a basis of the intertwiner space for functions on a 2-flower graph with $j_{1}=j_{2}=\frac{1}{2}$. Note that this is not an orthonormal basis, but by (5.40) have an angle of $\cos \frac{\pi}{3}$ with respect to each other. Now consider again the two-flower graph $\gamma=\left\{e_{1}, e_{2}\right\}$, but with $j_{1}=\frac{1}{2}, j_{2}=1$. Then again, the intertwiner space is two-dimensional, and one normalized vector is

$$
T_{3}(A):=\operatorname{tr}_{\frac{1}{2}}\left(A\left(e_{1}\right)\right) \operatorname{tr}_{1}\left(A\left(e_{2}\right)\right) .
$$

On the other hand, consider the automorphism $\phi^{\prime} \in \mathcal{A} u t(\mathcal{P})$ mapping the closed loop $e$ to $e_{1} \circ\left(e_{2}\right)^{2}$. One can then show that the projection of $\alpha_{\phi^{\prime}} T_{0}$ to the intertwiner space on the 2 -flower graph with $j_{1}=\frac{1}{2}, j_{2}=1$ is unequal $T_{3}$, but has non-vanishing inner product with $T_{3}$. Thus, this projection (denote it as $\Pi\left(\alpha_{\phi^{\prime}} T_{0}\right)$ ), together with $T_{3}$ form a basis for the intertwiner space for $j_{1}=\frac{1}{2}, j_{1}=1$. Again, this is no orthonormal basis, since the two vectors are only linearly independent, not orthogonal.

For each $E$-flower graph $\gamma=\left\{e_{1}, \ldots, e_{E}\right\}$ with spins $j_{1}, \ldots, j_{E}$ there is a normalized vector in the intertwiner space given by

$$
T_{E, \vec{j}}(A):=\prod_{k=1}^{E} \operatorname{tr}_{j_{k}}\left(A\left(e_{k}\right)\right) .
$$

In a graphical notation similar to the one used in the last chapter, we write

$$
\left[\begin{array}{llll}
\bigcirc_{j_{1}} & & & \\
& & & \bigcirc_{j_{2}} \\
& & \bigcirc_{j_{E-1}} & \\
& \bigcirc_{j_{E}} & & \cdots
\end{array}\right]:=\left[T_{E, \vec{j}}\right]
$$

Extending what we have just seen in the examples to higher flowers with higher spins suggests that all other elements in the intertwiner space can be composed by certain $\alpha_{\phi} T_{E^{\prime}, \vec{j}^{\prime}}$ with $E^{\prime}<E$. This would mean that the orbits $\left[T_{E, \vec{j}}\right]$ would constitute a basis for the set of orbits of all smooth cylindrical functions in $\mathcal{H}_{\text {kin }}$. 
Since these vectors have nontrivial overlap, however, one would have to compute their automorphism-invariant inner product by (5.35) and deduce an orthonormal basis from them by the Gram-Schmidt-procedure. This would provide a way to find an orthonormal basis for $\mathcal{H}_{\text {Aut }}$.

This would suggest that the linear combinations of the form

$$
\psi=\sum_{E=0}^{N} \sum_{j_{1}, \ldots, j_{E}} c_{E, \vec{j}}\left[\begin{array}{lll}
\bigcirc_{j_{1}} & & \\
& & \bigcirc_{j_{2}} \\
\bigcirc_{j_{E}} & & \ldots
\end{array}\right]
$$

form a dense set in $\mathcal{H}_{\text {Aut }}$. If one could then derive a good formula for the inner product between two such vectors, we would be able to write down an orthonormal basis for $\mathcal{H}_{\text {Aut }}$. So far, this has not been done due to the complicated combinatorics, but we will address this point in a later publication.

\section{Summary and Outlook}

\subsection{Summary of the work}

In this publication, we have investigated the consequence of extending the group of spatial diffeomorphisms $\operatorname{Diff}(\Sigma)$ to path groupoid automorphisms $\mathcal{A} u t(\mathcal{P})$ in Loop Quantum Gravity. This extension is inspired by category theory, and contains many elements that cannot be interpreted as diffeomorphisms from $\Sigma$ to itself. This mimics the extension of smooth to generalized gauge transformations: while the first consists of smooth maps from $\Sigma$ to the gauge group $G$, the latter one consists of all such maps, without continuity or even measurability assumption.

An automorphism is given by a permutation of the points in $\Sigma$, and a permutation of paths in $\Sigma$ which are compatible with each other in the sense that if the path $p$ starts at $x$ and ends at $y$, then the transformed path $\phi(p)$ starts at $\phi(x)$ and ends at $\phi(y)$. But by the groupoid structure of $\mathcal{P}$, the set of piecewise analytic paths in $\Sigma$, this does not necessarily restrict what happens to points that lie "in the middle" of $p$. So, the notion of a point lying on a path is not invariant under automorphisms, which makes many nontrivial constructions possible. In particular, some automorphisms simply cannot be interpreted as maps from $\Sigma$ to itself.

We have given some explicit examples for automorphisms that do not arise as diffeomorphisms on $\Sigma$. The first example was given by the natural transformations of the identity, which are able to arbitrarily permute the points in $\Sigma$, but keep the paths essentially the same (and which act, in particular, as identity on the gaugeinvariant part of the kinematical Hilbert space $\mathcal{H}_{\text {kin }}$ ). The second example was given by the edge-interchangers, which left all points invariant, but swapped two edges $e_{1}, e_{2}$ with the same beginning- and endpoints. All paths that meet these two edges at finitely many points are left invariant, however. In this sense, these automorphisms have support only at two edges $e_{1}, e_{2}$, and hence can be viewed as distributional.

We have used these two types of automorphisms in order to show that every two graphs with the same combinatorics can be mapped into each other by an 
automorphism $\phi \in \mathcal{A} u t(\mathcal{P})$. This shows how little information about the differential structure, and in fact even the topology of $\Sigma$ is encoded in the path groupoid $\mathcal{P}$.

We have delivered a proof that the action of $\mathcal{A} u t(\mathcal{P})$ on $\overline{\mathcal{A}}$, the set of (distributional) connections leave the Ashtekar-Isham-Lewandowski measure $\mu_{A L}$ invariant. This was straightforward in the case of the abelian gauge group $G=U(1)$, but nontrivial for arbitrary gauge groups.

In the last part of this work, we have investigated the induced action of the automorphisms $\mathcal{A} u t(\mathcal{P})$ on the kinematical Hilbert space $\mathcal{H}_{\text {kin }}$. In particular, we have showed that due to the size of automorphisms, the only information that is conserved by acting with automorphisms on a cylindrical function, is the combinatorics of paths, over which it is cylindrical. This information is highly redundant in the description of function cylindrical over paths, and it is the use of graphs to provide a way of finding a good representative in the set of collections of paths over which a function is cylindrical. Since the automorphisms do not leave the set of graphs invariant, functions cylindrical over one graph can be mapped to a function cylindrical over another graph, although the graphs themselves are not mapped to each other.

A gauge-invariant function on a graph $\gamma$ does only depend on the holonomies along a number of loops in $\gamma$, which correspond to the first fundamental class $\pi_{1}(\gamma)$. Consequently, any gauge-invariant function on a graph can be mapped to a gaugeinvariant function on some flower graph. This enabled us to gain some control over the size of the orbits of vectors in $\mathcal{H}_{\text {kin }}$ under the action of $\mathcal{A} u t(\mathcal{P})$. For some choice of rigging map, we derived the automorphism-invariant Hilbert space $\mathcal{H}_{\text {Aut }}$ for the gauge group $G=U(1)$. It was found that the space is infinite-dimensional and separable, the generic element being of the form

$$
\psi_{\text {Aut }}=\sum_{k=0}^{\infty} c_{n}\left[\bigcirc_{n}\right],
$$

with square-summable coefficients $\left\{c_{n}\right\}_{n \in \mathbb{N}}$, and $\left[\bigcirc_{n}\right]$ being the equivalence class of one Wilson loop with charge $n$.

For $G=S U(2)$, the combinatorics to work out the exact form of the orbits is harder, due to the recoupling scheme of $S U(2)$. However, we argued why we believe an element of the automorphism-invariant Hilbert space would be of the form

$$
\psi_{\text {Aut }}=\sum_{E=0}^{\infty} \sum_{j_{1}, \ldots, j_{E}} c_{E, \vec{j}}\left[\begin{array}{lll}
\bigcirc_{j_{1}} & & \\
& & \bigcirc_{j_{2}} \\
& \bigcirc_{j_{E-1}} & \\
\bigcirc_{j_{E}} & \ldots
\end{array}\right],
$$

where the vectors are equivalence classes of $E$ separate Wilson loops with spin charges $j_{k}$. These vectors will, however not be orthonormal to each other. Rather, their inner product will be determined by embedding-combinatorics of $E$-flowers into $E^{\prime}$-flowers for $E<E^{\prime}$, and the corresponding recoupling scheme. We will return to a detailed analysis of this space in a future publication. 


\subsection{Further directions}

By the form of the vectors (6.1) and (6.2), we see that the information about the degrees of freedom is completely delocalized, as one would have expected from a diffeomorphism-invariant theory. However, in order to get a good physical intuition for the meaning of the states in $\mathcal{H}_{\text {Aut }}$, one would have the following possibility: One can repeat the whole analysis, but with matter degrees of freedom coupled to gravity. Note that the automorphism-invariant content of a cylindrical function for pure gravity is just given by the combinatorics of paths, the holonomies of which it depends on, but not on how these paths are embedded into space, i.e. if they intersect, or are partially parallel. So the automorphism-invariant vectors do not know about the vertices of a graph, for instance. Having matter degrees of freedom coupled to gravity, this might change, due to the following reason: Consider a, say, fermionic matter field coupled to gravity. This field, when quantized along the lines of Loop Quantum Gravity, would become a field sitting on the vertices of a graph, and is transformed by some nontrivial representation of $S U(2)$. The information about which field is situated at which vertex will still be contained in the gauge-invariant sector of the theory. Shifting these gauge-invariant cylindrical functions for matter plus gravity around by automorphisms would result in graphs being mapped to other metagraphs, that look like different graphs, since the paths can intersect, or partially overlap. But that some matter is excited at some vertex will not be changed by this, so one would be able to distinguish the real vertices (where matter is excited) from the ones that just appear as vertices, because the paths are embedded into space in some peculiar way. This would provide a natural mechanism of how matter could be used to localize gravitational degrees of freedom, as has been advocated e.g. in [20].

Another important point is to see whether operators corresponding to physical observables can be defined, i.e. like the volume operator. This would not only allow for an interpretation of the automorphism-invariant states in terms of physical quantities. Also, if one could define a volume operator on the automorphism-invariant Hilbert space, one would be able to define the master constraint operator [21] on the automorphism-invariant Hilbert space. Since the graph-changing version of the master constraint changes in particular the first fundamental group of a graph, and the automorphism-invariant vectors contain exactly this information, this might provide a way to rephrase the quantum dynamics in some combinatorial way in $\mathcal{H}_{\text {Aut }}$. This would be particularly interesting, since one could make contact to [14], where such an operator already exists, leading to a combinatorial version of the dynamics.

One key step in this proof was theorem B.1, a classical result from combinatorial group theory by Nielsen ('36). On the other hand, we also relied on the explicit construction of the kinematical Hilbert space $\mathcal{H}_{\text {kin }}$ for this proof. From an aesthetical point of view, however, this is unsatisfactory, since we feel that the fundamental reason for the automorphisms to leave the measure $\mu_{A L}$ invariant is exactly Nielsen's theorem. It states a deep connection between automorphisms of free groups and the symmetries of the Haar measure. We are convinced that one can write down a proof that all automorphisms preserve $\mu_{A L}$, with only relying on Nielsen's theorem, without referring to the analytical structure of the manifold $\Sigma$ at all. This would 
enable us to transfer the proof to arbitrary groupoids $\mathcal{P}$, not necessarily the path groupoid of a spatial manifold $\Sigma^{9}$. With this one might be able to quantize much more general theories of connections on groupoids, not only on manifolds.

The path groupoid $\mathcal{P}$, as a category, is a useful concept when investigation quantizations of Riemannian metrics on 3-manifolds. It was indicated $[22,23,24]$ that for the investigation of Lorentzian metrics on 4-manifolds the notion of 2category is an appropriate concept. It would be interesting to investigate, in which sense the analysis presented here could be repeated in such a framework, in order to see space-time diffeomorphisms as automorphisms of 2-categories.

\section{Acknowledgements}

BB would like to thank Carla Cederbaum for time and patience, and Christian Fleischhack for discussions about hyphs, groupoids and analyticity. Also, thanks goes to Johannes Brunnemann, Andreas Döring, Cecilia Flori, Jerzy Lewandowski, Hendryk Pfeiffer and Hanno Sahlmann for numerous discussions about automorphisms.

\section{A Elements from category theory}

In this section, we will briefly review the basic notions of category theory that are used in this article ${ }^{10}$. Details and more about categories can be found in [25].

Definition A.1 A category $\mathcal{C}$ consists of a class of objects $X, Y, Z, \ldots$, denoted by $|\mathcal{C}|$, and, for each pair of objects $X, Y \in|\mathcal{C}|$ a class of morphisms $f, g, h, \ldots$, denoted by $\operatorname{Mor}_{\mathcal{C}}(X, Y)$. For these, the following rules hold:

- For each $f \in \operatorname{Mor}_{\mathcal{C}}(X, Y)$ and $g \in \operatorname{Mor}_{\mathcal{C}}(Y, Z)$ there is a morphism $g \circ f \in$ $\operatorname{Mor}_{\mathcal{C}}(X, Z)$ (the composite).

- Composition is associative, i.e. $h \circ(g \circ f)=(h \circ g) \circ f$.

- For each object $X \in|\mathcal{C}|$ there is a morphism $\operatorname{id}_{X} \in \operatorname{Mor}_{\mathcal{C}}(X, X)$ such that for all morphisms $f \in \operatorname{Mor}_{\mathcal{C}}(X, Y)$ and $g \in \operatorname{Mor}_{\mathcal{C}}(Z, X)$ one has

$$
f \circ \operatorname{id}_{X}=f, \quad \operatorname{id}_{X} \circ g=g
$$

If $f \in \operatorname{Mor}_{\mathcal{C}}(X, Y)$, then the source and the target of $f$ are denoted by $s(f):=X$ and $t(f):=Y$.

There are plenty of examples for categories:

- The category Set, the objects of which are sets, and the morphisms between two sets $X, Y$ are exactly all maps between these sets.

\footnotetext{
${ }^{9}$ At least as long as these groupoids are sets, since in these cases definitions of topology and measure make sense.

${ }^{10}$ Note that in this appendix, we use the standard convention for the composition, i.e. $f: X \rightarrow Y$ and $g: Y \rightarrow Z$ have a composition $g \circ f: X \rightarrow Z$. In the rest of the article, however, we will use the notation $f \circ g$ for the composition, in order to stay consistent with large parts of LQG literature.
} 
- For a manifold $\Sigma$, the category $\operatorname{Hom}(\Sigma)$, the objects of which are the points in $\Sigma$, and the morphisms of which are homotopy equivalence classes of curves between points.

- For a manifold $\Sigma$, the category $\mathcal{W}(\Sigma)$, the objects of which are points in $\Sigma$, and the morphisms between two points are all curves between these points, modulo reparametrization.

- For a group $G$, the category $\operatorname{Susp}(G)$, called the suspension of $G$. This category has only one object, denoted by $*$ : $|\operatorname{Susp}(G)|=\{*\}$, and the morphisms from $*$ to itself are in one-to-one correspondence with elements in $G$ : $\operatorname{Mor}_{\operatorname{Susp}(G)}(*, *)=G$.

A category $\mathcal{C}$ in which every morphism has a right and a left inverse is called a groupoid. In the above examples, Set and $\mathcal{W}(\Sigma)$ are no groupoids, but $\operatorname{Hom}(\Sigma)$ and $\operatorname{Susp}(G)$ are.

It is customary in category theory to write statements as diagrams: Consider a category $\mathcal{C}$ and objects $X, Y, Z, W \in|\mathcal{C}|$. Then the following diagram

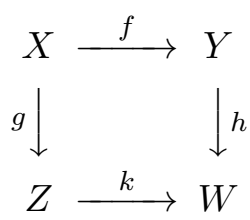

is said to commute, of $f \in \operatorname{Mor}_{\mathcal{C}}(X, Y), g \in \operatorname{Mor}_{\mathcal{C}}(X, Z), h \in \operatorname{Mor}_{\mathcal{C}}(Y, W)$ and $k \in \operatorname{Mor}_{\mathcal{C}}(Z, W)$, and $k \circ g=h \circ f$, as morphisms in $\operatorname{Mor}_{\mathcal{C}}(X, W)$. Working with commuting diagrams makes reasoning in category theory fairly intuitive. Since the proofs in this paper will be thoroughly analytical however, we will use commutative diagrams only at some points, to point out some connections.

Consider two morphisms $f, g$ in $\mathcal{W}(\Sigma)$ with $s(g)=t(f)$ can be composed, i.e. two curves (up to reparametrization) can be concatenated and again give a curve modulo parametrization. It should be noted that, due to the above definitions, the concatenation is denoted by $g \circ f$. However, in the Loop Quantum Gravity literature, this concatenation is usually denoted as $f \circ g$. The reason is that with this convention the generalized connections are functors from the path groupoid into the suspension of the gauge group $\operatorname{Susp}(G)$, not its opposite category $\operatorname{Susp}(G)^{o p}$.

This is just a matter of convention, of course, but it should be noted that, throughout this paper, the composition of morphisms $f, g$ will usually be denoted as $f \circ g$, not as $g \circ f$.

Definition A.2 Let $\mathcal{C}, \mathcal{D}$ be two categories. A functor is an assignment $F:|\mathcal{C}| \rightarrow$ $|\mathcal{D}|$ and $F: \operatorname{Mor}_{\mathcal{C}}(X, Y) \rightarrow \operatorname{Mor}_{\mathcal{D}}(F(X), F(Y))$, such that

- $F(g \circ f)=F(g) \circ F(f)$

- $F\left(i d_{X}\right)=i d_{F(X)}$

So a functor assigns objects to objects and morphisms to morphisms in a compatible way. As an example, consider the category which has smooth manifolds as objects 
and smooth maps between them as morphisms. Then the Cartan differential is a functor from this category in itself. In particular, let $f: M \rightarrow N$ and $g: N \rightarrow O$ be smooth maps, then the chain rule guarantees that

$$
d(g \circ f)=d g \circ d f
$$

as maps $d f: T M \rightarrow T N$, i.e. $d g: T N \rightarrow T O$.

Definition A.3 Let $F: \mathcal{C} \rightarrow \mathcal{D}$ and $G: \mathcal{C} \rightarrow \mathcal{C}$ be two functors. One calls these two functors to be related by a natural transformation (or being natural transformations from each other), if there is, for each object $X \in|\mathcal{C}|$ a morphism $g_{X} \in \operatorname{Mor}_{\mathcal{D}}(F(X), G(X))$ such that the following diagram commutes:

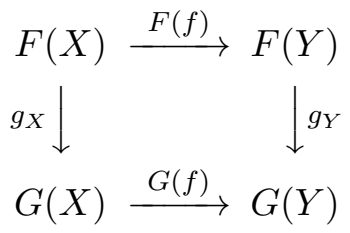

Note that, if $\mathcal{D}$ is a groupoid (i.e. its morphisms can be inverted), then given a functor $F$ and for each $X \in|\mathcal{C}|$ a morphism $g_{X} \in \operatorname{Mor}_{\mathcal{D}}(F(X), Y)$, then

$$
\begin{aligned}
G(X) & :=t\left(g_{X}\right) \quad \text { for all } X \in|\mathcal{C}| \\
G(f) & =g_{Y} \circ F(f) \circ g_{X}^{-1} \quad \text { for all } f \in \operatorname{Mor}_{\mathcal{C}}(X, Y)
\end{aligned}
$$

defines a functor $G$, which can be related to $F$ by a natural transformation. We will use this construction often for defining functors which are natural transformations of other, given functors.

\section{B Elements from combinatorial group theory}

Going over from piecewise analytic diffeomorphisms $\operatorname{Diff}(\Sigma)$ to the automorphisms $\mathcal{A} u t(\mathcal{P})$ is a significant enlargement of the gauge group. While the former preserves notions of (generalized) knotting classes of a graph, the latter one only keeps combinatorial information of the graph, i.e. which vertices are attached to each other by paths and which are not. Consequently, elements of combinatorial group theory enter the description, as soon as automorphisms are considered. In this section we review some basic notions from combinatorial group theory (details can be found in [26]) and conclude with a classical result of Nielson, which shows a connection between automorphisms of free groups and the symmetries of Haar measures. This will be one key point in proving that the elements of $\mathcal{A} u t(\mathcal{P})$ act unitarily on $\mathcal{H}_{\text {kin }}$.

Definition B.1 Let $E \in \mathbb{N}$, and $\left\{e_{1}, \ldots, e_{E}\right\}$ be $E$ abstract symbols, called letters. $A$ (finite) word in these letters is a (finite) sequence in these letters, i.e. $e_{2}, e_{4} e_{1}$ or $e_{1} e_{1} e_{7} e_{1}$. A word in the $2 E$ letters $\left\{e_{1}, \ldots, e_{E}, e_{1}^{-1}, \ldots, e_{E}^{-1}\right\}$ is called reduced, if no $e_{k}$ occurs next to its inverse $e_{k}^{-1}$. 
It is clear that every word can be put into its reduced form, by successively eliminating $e_{k} e_{k}^{-1}$ or $e_{k}^{-1} e_{k}$ from it.

Definition B.2 Consider the set of all reduced words in the $2 E$ letters $\left\{e_{1}^{ \pm 1}, \ldots, e_{E}^{ \pm 1}\right\}$, together with the "empty word", denoted by 1, and define multiplication between two words as concatenating (and possibly reducing) them. The set of all these reduced words form a group under this multiplication, which is called the free group in $E$ letters, and is denoted by $F_{E}$.

Examples:

$$
\begin{aligned}
& \left(e_{1} e_{2} e_{3}\right) \cdot\left(e_{3} e_{2} e_{1}\right)=e_{1} e_{2} e_{3} e_{3} e_{2} e_{1} \\
& \left(e_{1} e_{2} e_{3}\right) \cdot\left(e_{3}^{-1} e_{2}^{-1} e_{17}\right)=e_{1} e_{17}
\end{aligned}
$$

Similarly one can define the free group in zero $F_{0}=\{1\}$ and in countable many parameters $F_{\omega}$. Free groups play a prominent rôle in combinatorics, algorithm theory and graph theory. Obviously, for $E<E^{\prime}$, there is a natural inclusion of $F_{E}$ as a subgroup $F_{E^{\prime}}$. What is less intuitive is that the free group in two parameters $F_{2}$ has a subgroup isomorphic to $F_{\omega}$. As a consequence, every $F_{E}$ has a subgroup isomorphic to $F_{E^{\prime}}$ for $E<E^{\prime}$. This makes these groups more difficult than their linear counterparts, the $E$-dimensional vector spaces.

A homomorphism $\phi$ from a free group $F_{E}$ into another group is completely determined by its values on the basic letters, $\phi\left(e_{1}\right), \ldots, \phi\left(e_{E}\right)$. An invertible group homomorphism from $F_{E}$ to itself is called an automorphism, and is completely determined by the $E$ words $\phi\left(e_{k}\right)=\vartheta_{k}\left(e_{1}, \ldots, e_{E}\right)$ which are the images of the $e_{k}$ under $\phi$. The automorphisms of a free group are in fact well understood, which is shown by the following theorem.

Theorem B.1 (Nielsen) Let $\phi$ be an automorphism on $F_{E}$. Then $\phi$ can be written as a finite product of "elementary" automorphisms

$$
\phi=\xi_{n} \circ \xi_{n-1} \circ \ldots \circ \xi_{1}
$$

where every $\xi_{r}$ is one of the following:

- A permutation $(l \neq k \in\{1, \ldots, E\})$ :

$$
\xi_{r}\left(e_{k}\right)=e_{l}, \quad \xi_{r}\left(e_{l}\right)=e_{r}, \quad \xi_{r}\left(e_{m}\right)=e_{m} \text { else }
$$

- An inversion $(k \in\{1, \ldots E\})$ :

$$
\xi_{r}\left(e_{k}\right)=e_{k}^{-1}, \quad \xi_{r}\left(e_{l}\right)=\xi_{r}\left(e_{l}\right) \text { else }
$$

- $A$ shift $(k \neq l \in\{1, \ldots, E\})$ :

$$
\xi_{r}\left(e_{k}\right)=e_{k} e_{l}, \quad \xi_{r}\left(e_{l}\right)=\xi_{r}\left(e_{l}\right), \quad \xi_{r}\left(e_{m}\right)=e_{m} \text { else }
$$

This important structural theorem has an immediate consequence: 
Corollary B.1 Let $G$ be a compact Lie group with bi-invariant Haar measure $d \mu_{H}$. Let $F \in L^{1}\left(G^{E}, d \mu_{H}^{\otimes E}\right)$, and $\phi$ be an automorphism on $F_{E}$. Denote $\phi\left(e_{k}\right)=$ $\vartheta_{k}\left(e_{1}, \ldots, e_{E}\right)$. Then

$$
\begin{aligned}
\int_{G^{E}} d \mu_{H}^{\otimes E} & \left(h_{1}, \ldots, h_{E}\right) F\left(h_{1}, \ldots, h_{E}\right) \\
& =\int_{G^{E}} d \mu_{H}^{\otimes E}\left(h_{1}, \ldots, h_{E}\right) F\left(\vartheta_{1}\left(h_{1}, \ldots, h_{E}\right), \ldots, \vartheta_{E}\left(h_{1}, \ldots, h_{E}\right)\right),
\end{aligned}
$$

Proof: Nielson's theorem tells us that the substitution

$$
\begin{array}{cc}
h_{1} \longmapsto \vartheta_{1}\left(h_{1}, \ldots, h_{E}\right) \\
\vdots & \vdots \\
h_{E} & \longmapsto \\
& \vartheta_{E}\left(h_{1}, \ldots, h_{E}\right)
\end{array}
$$

can be achieved by successively applying the "elementary" substitutions (B.4), (B.5), (B.6) (with the symbols $h_{k}$, instead of the $e_{k}$ ). But these are exactly the substitutions under which the Haar measure $d \mu_{H}^{\otimes E}\left(h_{1}, \ldots, h_{E}\right)$ is invariant. The statement (B.7) follows.

\section{Categorical Weyl algebra of quantum grav- ity}

The holonomies and their transformations, i.e. gauge-transformations and diffeomorphisms, can be formulated in terms of category theory, and this formulation suggests to enlarge the diffeomorphism group $\operatorname{Diff}(\Sigma)$ to the automorphisms of the path groupoid $\mathcal{A} u t(\mathcal{P})$, which act unitarily on the kinematical Hilbert space $\mathcal{H}_{\text {kin }}$ of Loop Quantum Gravity.

The field algebra of Loop quantum gravity, however, consists of holonomies as well as fluxes, and the symmetry groups act on both. In this section, we will show that also the fluxes can be formulated naturally in the languages of categories ${ }^{11}$.

We will work with the exponentiated fluxes instead of the fluxes themselves, which leads to the Weyl algebra of LQG, for technical reasons. The fluxes themselves can be recovered from these exponentiated versions easily.

\section{C.1 Categorial formulation of oriented surfaces}

We want to obtain the categorical formulation of an "oriented surface". An oriented surface does in principle two things: First, it cuts a path into several pieces (wherever a path intersects the surface), and second, it assigns to each of these pieces two numbers in $\{-1,0,1\}$. These numbers depend on whether the the startingor, respectively, the end point of the piece meets the surface along or against the orientation of the surface $( \pm 1)$, or whether the starting- or end part of the piece lies

\footnotetext{
${ }^{11}$ Again, we use the convention that the concatenation of two morphisms $p$ and $q$ is denoted as $p \circ q$ if $t(p)=s(q)$, in order to stay consistent with the notation in [4]
} 
entirely away from, or entirely within the surface (in which case it gets assigned 0). We will now turn this intuition into a categorical language.

Definition C.1 Let $\mathscr{C}$ be a category. Then define the category $\mathcal{O} r \mathcal{C} u(\mathscr{C})$ as follows:

- The objects in both categories are the same: $\mid \mathcal{O}$ Cut $(\mathscr{C})|=| \mathscr{C} \mid$.

- Let $X, Y$ be objects in $\mathcal{O}$ Cut $(\mathscr{C})$. The morphisms $\operatorname{Mor}(X, Y)$ in $\operatorname{OrCut}(\mathscr{C})$ can be constructed as follows: Consider three finite sequences with all the same length $N$ : A sequence of paths $p_{1}, \ldots, p_{N}$, as well as two sequences of natural numbers $m_{1}, \ldots, m_{N}$ and $n_{1}, \ldots n_{N}$. We arrange these three sequences as

$$
\left(\begin{array}{c}
p_{1}, \ldots, p_{N} \\
m_{1}, \ldots, m_{N} \\
n_{1}, \ldots, n_{N}
\end{array}\right)
$$

The paths have the properties that $t\left(p_{k}\right)=s\left(p_{k+1}\right)$ for $k=1, \ldots N-1$, as well as $s\left(p_{1}\right)=X$ and $t\left(p_{N}\right)=Y$. We define an equivalence relation on these three sequences, by

$$
\left(\begin{array}{c}
p_{1}, \ldots, p_{k}, p_{k+1}, \ldots, p_{N} \\
m_{1}, \ldots, m_{k},-n, \ldots, m_{N} \\
n_{1}, \ldots, n, n_{k+1}, \ldots, n_{N}
\end{array}\right) \sim\left(\begin{array}{ccc}
p_{1}, \ldots, p_{k} \circ p_{k+1}, \ldots, p_{N} \\
m_{1}, \ldots, & m_{k}, & \ldots, m_{N} \\
n_{1}, \ldots, & n_{k+1}, & \ldots, n_{N}
\end{array}\right)
$$

and

$$
\left(\begin{array}{c}
p_{1}, \ldots, \mathrm{id}, \ldots, p_{N} \\
m_{1}, \ldots, m, \ldots, m_{N} \\
n_{1}, \ldots, n, \ldots, n_{N}
\end{array}\right) \sim\left(\begin{array}{c}
p_{1}, \ldots, \quad \mathrm{id}, \ldots, p_{N} \\
m_{1}, \ldots, m+k, \ldots, m_{N} \\
n_{1}, \ldots, n-k, \ldots, n_{N}
\end{array}\right)
$$

for all $k \in \mathbb{Z}$. The morphisms from $X$ to $Y$ then contain all equivalence classes of these sequences, which we denote by

$$
\left[\begin{array}{c}
p_{1}, \ldots, p_{N} \\
m_{1}, \ldots, m_{N} \\
n_{1}, \ldots, n_{N}
\end{array}\right] \in \operatorname{Mor}(X, Y)
$$

Concatenation of morphisms is obtained by just concatenating the sequences:

$$
\left[\begin{array}{c}
p_{1}, \ldots, p_{N} \\
m_{1}, \ldots, m_{N} \\
n_{1}, \ldots, n_{N}
\end{array}\right] \circ\left[\begin{array}{c}
q_{1}, \ldots, q_{M} \\
r_{1}, \ldots, r_{M} \\
s_{1}, \ldots, s_{M}
\end{array}\right]:=\left[\begin{array}{c}
p_{1}, \ldots, p_{N}, q_{1}, \ldots, q_{M} \\
m_{1}, \ldots, m_{N}, r_{1}, \ldots, r_{M} \\
n_{1}, \ldots, n_{N}, s_{1}, \ldots, s_{M}
\end{array}\right]
$$

while the identity functor in $\operatorname{Mor}(X, X)$ is given by

$$
\text { id }:=\left[\begin{array}{c}
\operatorname{id}_{X} \\
0 \\
0
\end{array}\right]
$$


It is straightforward to check that with these equivalence relations, the category $\mathcal{O} \operatorname{C} u t(\mathscr{C})$ becomes a groupoid, if $\mathscr{C}$ is one, if one defines the inverse of a morphism by

$$
\left[\begin{array}{c}
p_{1}, \ldots, p_{N} \\
m_{1}, \ldots, m_{N} \\
n_{1}, \ldots, n_{N}
\end{array}\right]^{-1}:=\left[\begin{array}{c}
p_{N}^{-1}, \ldots, p_{1}^{-1} \\
-n_{N}, \ldots,-n_{1} \\
-m_{N}, \ldots,-m_{1}
\end{array}\right]
$$

There is a projection functor

$$
\pi: \mathcal{O r C u t}(\mathscr{C}) \rightarrow \mathscr{C}
$$

the action of which is given by the identity on the objects, and by

$$
\pi:\left[\begin{array}{c}
p_{1}, \ldots, p_{N} \\
m_{1}, \ldots, m_{N} \\
n_{1}, \ldots, n_{N}
\end{array}\right] \longmapsto p_{1} \circ \cdots \circ p_{N} .
$$

One checks quickly that this action is well-defined.

In the following, we will consider the path groupoid $\mathcal{P}$ and the category $\operatorname{Or} \mathcal{C} u t(\mathcal{P})$. With the category $\operatorname{OrCut}(\mathcal{P})$ we have a notion at hand to say what "cutting an edge" $p$ means in category language.

Definition C.2 Let $\mathcal{P}$ be the path groupoid of a manifold $\Sigma$. Then a functor

$$
S: \mathcal{P} \longrightarrow \mathcal{O r C u t}(\mathcal{P})
$$

is called a generalized oriented surface, if the following two conditions hold:

- The functor $\pi \circ S$ is the identity functor on $\mathcal{P}$.

- For each primitive metagraph $\mu$ with morphisms $q_{1}, \ldots, q_{n}$ in $\mathcal{P}$, there is a primitive metagraph $\mu^{\prime} \geq \mu$ with morphisms $p_{1}, \ldots, p_{m}$, such that each $p_{k}$ is $S$-trivial, which means that

$$
S\left(p_{k}\right)=\left[\begin{array}{c}
p_{k} \\
m_{k} \\
n_{k}
\end{array}\right]
$$

for some natural numbers $n_{l}, m_{l}$.

The interpretation of the first condition is obvious: To each edge $p, S$ assigns a collection $p_{1}, \ldots, p_{N}$ such that $p_{1} \circ \cdots \circ p_{N}=p$, i.e. it cuts the path into pieces. Furthermore, to each piece there are assigned two natural numbers, which determine the position of the ends of the piece with respect to $S$. It is obvious that each oriented surface in $\Sigma$, and also each quasi-surface together with an orientation function in the sense of [27] determines a generalized surface in the above sense. 
The second condition ensures that the surface does not cut a path in a too "wild" way, and in particular cuts several different paths consistently with each other. It is this condition that will ensure that the Weyl operators constructed from the generalized oriented surfaces will be unitarities.

Definition C.3 Let $\Sigma$ be a manifold and $\mathcal{P}$ its path groupoid. Let $G$ be a compact Lie group and $\overline{\mathcal{A}}$ be the set of all functors from $\mathcal{P}$ to $\operatorname{Susp}(G)$. Let furthermore $S$ be a generalized oriented surface and $d: \Sigma \rightarrow G$ be a map.

Then there is a transformation of functors

$$
\Theta_{S, d}: \overline{\mathcal{A}} \longrightarrow \overline{\mathcal{A}}
$$

which is given by the following rule: If

$$
S(p)=\left[\begin{array}{c}
p_{1}, \ldots, p_{N} \\
m_{1}, \ldots, m_{N} \\
n_{1}, \ldots, n_{N}
\end{array}\right]
$$

then

$$
\begin{aligned}
\Theta_{S, d} A(p) & :=\prod_{k=1 \ldots N} d\left(s\left(p_{k}\right)\right)^{m_{k}} A\left(p_{k}\right) d\left(t\left(p_{k}\right)\right)^{n_{k}} \\
& :=d\left(s\left(p_{1}\right)\right)^{m_{1}} A\left(p_{1}\right) d\left(t\left(p_{1}\right)\right)^{n_{1}} \cdot \ldots \cdot d\left(s\left(p_{N}\right)\right)^{m_{N}} A\left(p_{N}\right) d\left(t\left(p_{N}\right)\right)^{n_{N}} .
\end{aligned}
$$

It is easy to check that this action is well-defined, i.e. does not depend on the representant of the equivalence class $S(p)$. One can also readily see that this action coincides with the action of the Weyl elements determined by oriented quasi-surfaces $[27]$.

Lemma C.1 Let $S$ be a generalized oriented surface and $d: \Sigma \rightarrow G$ be a map. Then $\Theta_{S, d}$ given by definition C.3 has the following properties:

i) $\alpha_{\phi} \circ \Theta_{S, d} \circ \alpha_{\phi^{-1}}=\Theta_{\alpha_{\phi} S, d \circ \phi^{-1}}$

ii) $\Theta_{S, d}$ is continuous on $\overline{\mathcal{A}}$.

iii) $\Theta_{S, d}$ leaves the Ashtekar-Isham-Lewandowski measure invariant, i.e. $\Theta_{S, d}^{*} \mu_{A L}=$ $\mu_{A L}$.

\section{Proof:}

i) Each automorphism $\phi \in \mathcal{A} u t(\mathcal{P})$ acts naturally on the set of generalized oriented surfaces, by

$$
S(p)=\left[\begin{array}{c}
p_{1}, \ldots, p_{N} \\
m_{1}, \ldots, m_{N} \\
n_{1}, \ldots, n_{N}
\end{array}\right] \Rightarrow \alpha_{\phi} S(\phi(p))=\left[\begin{array}{ccc}
\phi\left(p_{1}\right), \ldots, \phi\left(p_{N}\right) \\
m_{1}, \ldots, & m_{N} \\
n_{1}, \ldots, & n_{N}
\end{array}\right](\mathrm{C} .15)
$$


By direct computation we obtain

$$
\begin{aligned}
\alpha_{\phi} \circ \Theta_{S, d} \circ \alpha_{\phi}^{-1} A(\phi(p)) & =\Theta_{S, d} \circ \alpha_{\phi}^{-1} A(p) \\
& =\prod_{k=1, \ldots, N} d\left(s\left(p_{k}\right)\right)^{m_{k}}\left(\alpha_{\phi}^{-1} A\right)\left(p_{k}\right) d\left(t\left(p_{k}\right)\right)^{n_{k}} \\
& =\prod_{\prod_{k=1, \ldots, N}}\left(d \circ \phi^{-1}\right)\left(s\left(\phi\left(p_{k}\right)\right)\right)^{m_{k}} A\left(\phi\left(p_{k}\right)\right)\left(d \circ \phi^{-1}\right)\left(t\left(\phi\left(p_{k}\right)\right)\right)^{n_{k}} \\
& =\Theta_{\alpha_{\phi} S, d_{\phi^{-1}}} A(\phi(p))
\end{aligned}
$$

Since $\phi$ is an automorphism of $\mathcal{P}$, it follows that

$$
\alpha_{\phi} \circ \Theta_{S, d} \circ \alpha_{\phi}^{-1}=\Theta_{\alpha_{\phi} S, d \circ \phi^{-1}} .
$$

ii) Since for each primitive metagraph $\mu$ there is a finer primitive metagraph $\mu^{\prime} \geq \mu$ which consists only of $S$-trivial morphisms, the set of such metagraphs is a partially ordered, directed set. As a consequence, the set of all

$$
\pi_{\mu^{\prime}}^{-1}\left(U_{1} \times \ldots \times U_{|E(\mu)|}\right) \subset \overline{\mathcal{A}}
$$

constitutes a basis for the topology on $\overline{\mathcal{A}}$, if the $U_{k} \subset G$ are open.

iii) Let $\mu=\left\{p_{1}, \ldots, p_{M}\right\}$ be a primitive metagraph and $f \in C y l(\mu)$ smooth and cylindrical over $\mu$. Then

$$
\int_{\overline{\mathcal{A}}} d \mu_{A L}(A) f(A)=\int_{G^{E}} d \mu_{H}\left(h_{1}, \ldots, h_{M}\right) F\left(h_{1}, \ldots, h_{M}\right)
$$

for some smooth function $F$ on $G^{M}$. Let $\mu^{\prime}=\left\{q_{1}, \ldots, q_{M^{\prime}}\right\}$ be a metagraph finer than $\mu$ with $S$-trivial paths $q_{k}$. For each $p_{k} \in \mu$ we can then find paths $q_{1}^{k}, \ldots, q_{n_{k}}^{k} \in \mu^{\prime}$, such that

$$
S\left(p_{k}\right)=\left[\begin{array}{c}
q_{1}^{k}, \ldots, q_{n_{k}}^{k} \\
m_{1}^{k}, \ldots, m_{n_{k}}^{k} \\
n_{1}^{k}, \ldots, n_{n_{k}}^{k}
\end{array}\right]
$$

Then by (C.14), $\Theta_{S, d} f$ is cylindrical over $\mu^{\prime}$, and one has

$$
\begin{aligned}
& \int_{\overline{\mathcal{A}}} d \mu_{A L}(A) \Theta_{S, d} f(A)=\int_{G^{M^{\prime}}} d \mu_{H}\left(h_{1}, \ldots, h_{M^{\prime}}\right) \\
& F\left(\prod_{l=1 \ldots n_{1}} d\left(s\left(q_{l}^{1}\right)\right)^{m_{l}^{1}} h_{l}^{1} d\left(t\left(q_{l}^{1}\right)\right)^{n_{l}^{1}}, \ldots, \prod_{l=1 \ldots n_{M}} d\left(s\left(q_{l}^{M}\right)\right)^{m_{l}^{M}} h_{l}^{M} d\left(t\left(q_{l}^{M}\right)\right)^{n_{l}^{M}}\right)
\end{aligned}
$$

where the $h_{l}^{k}$ are the $h_{m}$ corresponding to the decomposition $p_{k}=q_{1}^{k} \circ \ldots \circ q_{n_{k}}^{k}$ given by (C.20). Since the integration in (C.21) uses the bi-invariant Haar measure on $G^{M^{\prime}}$, we can perform a shift of integration variables

$$
h_{l}^{k} \longrightarrow d\left(s\left(q_{l}^{k}\right)\right)^{-m_{l}^{k}} h_{l}^{k} d\left(t\left(q_{l}^{k}\right)\right)^{-n_{l}^{k}}
$$


which gives us

$$
\begin{aligned}
\int_{\overline{\mathcal{A}}} d \mu_{A L}(A) \Theta_{S, d} f(A) & =\int_{G^{M^{\prime}}} d \mu_{H}\left(h_{1}, \ldots, h_{M^{\prime}}\right) F\left(h_{1}^{1} \cdots h_{n_{1}}^{1}, \ldots, h_{1}^{M} \cdots h_{n_{M}}^{M}\right) \\
& =\int_{G^{M}} d \mu_{H}\left(h_{1}, \ldots, h_{M}\right) F\left(h_{1}, \ldots, h_{M}\right) \quad(\mathrm{C} .23) \\
& =\int_{A L} d \mu_{A L}(A) f(A),
\end{aligned}
$$

since $\mu \leq \mu^{\prime}$ is an inclusion of primitive subgraphs, and the integral of $f$ over $\overline{\mathcal{A}}$ does not depend on the choice of primitive metagraph over which it is cylindrical. Thus, we have

$$
\int_{\overline{\mathcal{A}}} d \mu_{A L}(A) \Theta_{S, d} f(A)=\int_{A L} d \mu_{A L}(A) f(A)
$$

for all cylindrical functions $f$, which form a dense subset in $C(\overline{\mathcal{A}})$, and since $\Theta_{S, d}$ acts continuously on $\overline{\mathcal{A}}$, we have that (C.24) leaves $\mu_{A L}$ invariant.

The action of $\Theta_{S, d}$ can naturally be pulled back to $\mathcal{H}_{\text {kin }}=L^{2}\left(\overline{\mathcal{A}}, d \mu_{A L}\right)$ by, where they act as unitary operators $\hat{U}(S, d)$. These operators generalize the exponentiated fluxes that one can build from surfaces and smooth fields $E_{I}^{a}$ on $\Sigma$. In fact, one can recover generalized fluxes $E(S)$ from the $\Theta_{S, d}$.

Lemma C.2 Let $\Sigma$ be a manifold, $G$ be a compact Lie group, $S$ be a generalized oriented surface, and $d_{1}, d_{2}: \Sigma \rightarrow G$ two pointwise commuting maps, i.e. $d_{1}(x) d_{2}(x)=d_{2}(x) d_{1}(x)$ for all $x \in \Sigma$. Then

$$
\Theta_{S, d_{1}} \circ \Theta_{S, d_{2}}=\Theta_{S, d_{1} d_{2}}
$$

Proof: By direct calculation. Let $p$ be a path in $\Sigma$, then by the definition of $S$, there are $S$-trivial paths $p_{1}, \ldots, p_{N}$ with $p=p_{1} \circ \cdots \circ p_{N}$ and numbers $m_{1}, \ldots, m_{N}$, $n_{1}, \ldots, n_{N}$ such that

$$
S\left(p_{k}\right)=\left[\begin{array}{c}
p_{k} \\
m_{k} \\
n_{k}
\end{array}\right]
$$

Since $S$ is a functor, we have

$$
S(p)=\left[\begin{array}{c}
p_{1}, \ldots, p_{N} \\
m_{1}, \ldots, m_{N} \\
n_{1}, \ldots, n_{N}
\end{array}\right]
$$

Thus we see that one can choose a representant of $S(p)$ contains $S$-trivial paths only. We now have 


$$
\begin{aligned}
\Theta_{S, d_{1}} \circ \Theta_{S, d_{2}} A(p) & =\prod_{k=1 \ldots N} d_{1}\left(s\left(p_{k}\right)\right)^{m_{k}}\left(\Theta_{S, d_{2}} A\right)\left(p_{k}\right) d_{1}\left(t\left(p_{k}\right)\right)^{n_{k}} \\
& =\prod_{k=1 \ldots N} d_{1}\left(s\left(p_{k}\right)\right)^{m_{k}}\left(d_{2}\left(s\left(p_{k}\right)\right)^{m_{k}} A\left(p_{k}\right) d_{2}\left(t\left(p_{k}\right)\right)^{n_{k}}\right) d_{1}\left(t\left(p_{k}\right)\right)^{n_{k}} \\
& =\prod_{k=1 \ldots N}\left(d_{1} d_{2}\right)\left(s\left(p_{k}\right)\right)^{m_{k}} A\left(p_{k}\right)\left(d_{2} d_{1}\right)\left(t\left(p_{k}\right)\right)^{n_{k}} \\
& =\Theta_{S, d_{1} d_{2}} A(p)
\end{aligned}
$$

since $d_{1}$ and $d_{2}$ commute pointwise. Here the property that all $p_{k}$ are $S$-trivial was essential for the proof.

We now show that a generalized flux can be obtained by differentiating the action of the exponentiated fluxes.

Lemma C.3 Let $\Sigma$ be a manifold, $G$ be a compact Lie group, $S$ a generalized oriented surface and $k: \Sigma \rightarrow \mathfrak{g}$ be a map from $\Sigma$ to the Lie algebra of $G$. Then the operator

$$
E_{k}(S):=-\left.i \frac{d}{d t} \hat{U}\left(S, e^{i t k}\right)\right|_{t=0}
$$

defines a self-adjoint operator on $\mathcal{H}_{\mathrm{kin}}$, the domain of definition of which contains the cylindric functions $C y l$ on $\overline{\mathcal{A}}$.

Proof: Lemma C.2 shows that

$$
\Theta_{S, e^{i t k}} \circ \Theta_{S, e^{i s k}}=\Theta_{S, e^{i(t+s) k}},
$$

i.e. the map $t \longmapsto \hat{U}\left(S, e^{i t k}\right)$ defines a one-parameter family of unitarities on $\mathcal{H}_{\text {kin }}$. Thus, the Stone-von-Neumann theorem guarantees the existence of (C.30), if the limits of matrix elements

$$
\lim _{t \rightarrow 0}\left\langle f\left|\hat{U}\left(S, e^{i t k}\right)\right| f^{\prime}\right\rangle=\left\langle f \mid f^{\prime}\right\rangle
$$

exist for all $f, f^{\prime}$ being in a dense set $D \subset \mathcal{H}_{\text {kin }}$. Let $f, f^{\prime} \in C y l$ be two smooth cylindrical functions over a metagraph $\mu$, which can, without loss of generality be chosen to be the same for $f$ and $f^{\prime}$. Then there is, by definition of $S$, a metagraph $\mu^{\prime} \geq \mu$ consisting of $S$-trivial morphisms $q_{1}, \ldots, q_{M}$. The functions $f$ and $f^{\prime}$ are then obviously also cylindrical over $\mu^{\prime}$, i.e. there are smooth functions $F, F^{\prime}: G \longrightarrow \mathbb{C}$ such that

$$
\begin{aligned}
f(A) & =F\left(A\left(q_{1}\right), \ldots, A\left(q_{M}\right)\right) \\
f^{\prime}(A) & =F^{\prime}\left(A\left(q_{1}\right), \ldots, A\left(q_{M}\right)\right)
\end{aligned}
$$


Hence, since $S\left(q_{l}\right)=\left[\begin{array}{c}q_{l} \\ m_{l} \\ n_{l}\end{array}\right]$, we have

$$
\begin{aligned}
\left(U\left(S, e^{i t k}\right) f^{\prime}\right)(A) & =f^{\prime}\left(\Theta_{S, e^{i t k}} A\right) \\
& =F^{\prime}\left(e^{i t m_{1} k_{1}^{s}} A\left(q_{1}\right) e^{i t n_{1} k_{1}^{t}}, \ldots, e^{i t m_{M} k_{M}^{s}} A\left(q_{M}\right) e^{i t n_{M} k_{M}^{t}}\right)
\end{aligned}
$$

with $k_{l}^{s}:=k\left(s\left(q_{l}\right)\right) \in \mathfrak{g}$ and $k_{l}^{t}=k\left(t\left(q_{l}\right)\right) \in \mathfrak{g}$. From this it follows that

$$
\left\langle\psi\left|U\left(S, e^{i t k}\right)\right| \phi\right\rangle=\int_{G^{M}} d \mu_{H}\left(h_{1}, \ldots, h_{M}\right) \overline{F\left(h_{1}, \ldots, h_{M}\right)} F^{\prime}\left(e^{i t m_{1} k_{1}^{s}} h_{1} e^{i t n_{1} k_{1}^{t}}, \ldots, e^{i t m_{M} k_{M}^{s}} h_{M} e^{i t n_{M} k_{M}^{t}}\right) .
$$

Since $F, F^{\prime}$ are smooth and $G$ is compact, the expression is smooth in $t$. Since the smooth cylindrical functions $C y l$ over metagraphs are dense in $\mathcal{H}_{\text {kin }}$, the claim follows from the Stone-von-Neumann theorem.

\section{C.2 Algebra relations}

The smooth cylindrical functions $f \in C y l$ and the maps $\Theta_{S, d}$ generate an algebra, which can be completed to a $C^{*}$-algebra $\mathfrak{A}_{\text {cat }}$, similar to the Weyl algebra of quantum geometry [10]. Cyl, which is an abelean subalgebra of $\mathfrak{A}_{\text {cat }}$ is generated by functions cylindrical over just one morphism. Let $f$ be a function cylindrical over $p$, i.e. there is a smooth function $F: G \rightarrow \mathbb{C}$ with $f(A)=F(A(p))$. Let furthermore

$$
S(p)=\left[\begin{array}{c}
p_{1}, \ldots, p_{N} \\
m_{1}, \ldots, m_{N} \\
n_{1}, \ldots, n_{N}
\end{array}\right]
$$

The function $f$ acts via multiplication on, say, $C(\overline{\mathcal{A}})$. Then, for any $g \in C(\overline{\mathcal{A}})$, we have

$$
\begin{aligned}
\Theta_{S, d} f \Theta_{S, d}^{-1} g(A) & =\left(f \Theta_{S, d}^{-1} g\right)\left(\Theta_{S, d} A\right) \\
& =f\left(\Theta_{S, d} A\right) \cdot \Theta_{S, d}^{-1} g\left(\Theta_{S, d} A\right) \\
& =f\left(\Theta_{S, d} A\right) \cdot g(A)
\end{aligned}
$$

which gives

$$
\Theta_{S, d} f \Theta_{S, d}^{-1}=f \circ \Theta_{S, d},
$$

which is the usual algebraic relation which also determines the Weyl algebra of quantum geometry. To cast (C.34) into a more frequently used form, we go over to the (generalized) fluxes $E_{f}(S)$. As operators on $\mathcal{H}_{\text {kin }},(\mathrm{C} .34)$ reads

$$
\hat{U}(S, d) \hat{f} \hat{U}(S, d)^{-1}=\widehat{f \circ \Theta_{S, d}}
$$


where $\hat{f}$ is the operator corresponding to multiplication with $f \in C y l$ on $\mathcal{H}_{\text {kin }}$. For $k: \Sigma \rightarrow \mathfrak{g}$ any map, we get, by lemma C.3

$$
-i \frac{d}{d t}\left[\hat{U}\left(S, e^{i t k}\right) \hat{f} \hat{U}\left(S, e^{i t k}\right)^{-1}\right]_{t=0}=\left[\hat{E}_{k}(S), \hat{f}\right]
$$

On the other hand, the derivative of the function $f \circ \Theta_{S, e^{i t k}}$ with respect to $t$ reads

$$
\begin{aligned}
-\left.i \frac{d}{d t}\left[f\left(\Theta_{S, e^{i t k}} A\right)\right]\right|_{t=0} & =-\left.i \frac{d}{d t}\left[\prod_{l=1, \ldots, N} e^{i t m_{l} k_{l}^{s}} A\left(p_{l}\right) e^{i t n_{l} k_{l}^{t}}\right]\right|_{t=0} \\
& =\tilde{F}\left(A\left(p_{1}\right), \ldots, A\left(p_{N}\right)\right)
\end{aligned}
$$

where $k_{l}^{s}=k\left(s\left(p_{l}\right)\right), k_{l}^{t}=k\left(t\left(p_{l}\right)\right) \in \mathfrak{g}$. The function $\tilde{F}: G^{N} \rightarrow \mathbb{C}$ is given by

$$
\tilde{F}\left(h_{1}, \ldots, h_{N}\right)=\sum_{l=0}^{N}\left(n_{l}+m_{l+1}\right) F\left(h_{1} \cdot \ldots \cdot k_{l} k_{l}^{t} h_{l+1} \cdot \ldots \cdot h_{N}\right) .
$$

In this notation, $k_{0}^{t}:=k_{1}^{s}=k(s(p))$. Furthermore $n_{0}:=m_{N+1}:=0$, also $h_{1} \cdot \ldots$. $h_{0}:=\mathbb{1}$, as well as $h_{N+1} \cdot \ldots \cdot h_{N}:=\mathbb{1}$. We thus get

$$
\left[\hat{E}_{k}(S), \hat{f}\right](A)=\sum_{l=0}^{N} \varepsilon\left(S, p_{l}\right) F\left(A\left(p_{1}\right) \cdot \ldots \cdot A\left(p_{l}\right) k_{l}^{t} A\left(p_{l+1}\right) \cdot \ldots \cdot A\left(p_{N}\right)(C .39)\right.
$$

Here $\varepsilon\left(S, p_{l}\right):=n_{l}+m_{l+1}$. If $S$ is an analytic surface and $p$ is an edge, this formula reduces to the one employed in the literature (modulo prefactors as $l_{p}^{2}$ and the Immirzi parameter, which can be absorbed into the definition of $\left.E_{k}(S)\right)$. We have

$$
S(p)=\left[\begin{array}{c}
p_{1}, \ldots, p_{N} \\
m_{1}, \ldots, m_{N} \\
n_{1}, \ldots, n_{N}
\end{array}\right]
$$

so $S$ cuts the edge $p$ into the pieces $p_{1}, \ldots, p_{N}$, and thus the $k_{l}^{t}, l=0, \ldots, N$ are the values of the smearing function $k$ at the endpoints of $p$ and the points where $S$ intersects $p$. At these points, the values $k_{l}^{t} \in \mathfrak{g}$ are inserted in the arguments of the function $F$, and the sum is taken over all these points, with prefactors $\left(n_{l}+m_{l+1}\right)$. If one restricts the values of the intersection functions $m_{l}, n_{l}$ to $\{0, \pm 1\}$, then the factor $\varepsilon\left(S, p_{l}\right)$ keeps track of the way in which the segment $p_{l}$ touches $S$ at $s\left(p_{l}\right) \in \Sigma$.

Note that, since $S(p)$ is an equivalence class, so strictly speaking $\tilde{F}$ depends on the choice of the representant. With the precise definition of $S(p)$ however, one can easily see that the function $\tilde{f}(A)=\tilde{F}\left(A\left(p_{1}\right), \ldots, A\left(p_{N}\right)\right)$ does not.

This shows that (C.39) generalizes the canonical commutation relations for quantum gravity to a $*$-algebra obtained from $\mathfrak{A}_{\text {cat }}$, which is generated by smooth cylindrical functions $f$ over metagraphs and generalized electric fluxes $E_{k}(S)$. 


\section{References}

[1] Ashtekar, A., Lewandowski, J., Marolf, D., Mourao, J., Thiemann, T. Quantization of diffeomorphism invariant theories of connections with local degrees of freedom 1995 J. Math. Phys. 366456 [arXiv:gr-qc/9504018]

[2] Rovelli, C.: Quantum Gravity (Cambridge Monographs on Mathematical Physics) Cambridge University Press 2004

[3] Smolin, L.: An invitation to Loop Quantum Gravity [arXiv:hep-th/0408048]

[4] Thiemann, T.: Modern Canonical Quantum General Relativity (Cambridge Monographs on Mathematical Physics) Cambridge University Press 2007

[5] Fairbairn, W., Rovelli, C.: Separable Hilbert space in Loop Quantum Gravity 2004 J.Math.Phys. 45 2802-2814 [arXiv:gr-qc/0403047]

[6] Koslowski, T.: Physical Diffeomorphisms in Loop Quantum Gravity [arXiv:grqc/0610017]

[7] Ashtekar, A., Lewandowski, J.: Background Independent Quantum Gravity: A Status Report 2004 Class.Quant.Grav. 21 R53 [arXiv:gr-qc/0404018]

[8] Lewandowski, J., Okolow, A., Sahlmann, H., Thiemann, T. Uniqueness of diffeomorphism invariant states on holonomy-flux algebras 2006 Commun. Math. Phys. 267 No. 3, 703 [arXiv:gr-qc/0504147]

[9] Zapata, J.A:: Combinatorial space from Loop Quantum Gravity, 1998 Gen.Rel.Grav. 30 1229-1245 [arXiv:gr-qc/9703038]

[10] Fleischhack, C.: Representations of the Weyl algebra in quantum geometry [arXiv:math-ph/0407006]

[11] Baez, J.: Spin Networks in Gauge Theory 1996 Adv. Math. 117, 253-272

[12] Velhinho, J. M.: Functorial aspects of the space of generalized connections 2005 Mod.Phys.Lett. A20 1299 [arXiv:math-ph/0411073]

[13] Zapata, J.A.: A combinatorial approach to diffeomorphism invariant quantum gauge theories 1997 J. Math. Phys. 385663 [arXiv:gr-qc/9703037]

[14] Giesel, K., Thiemann, T.Algebraic Quantum Gravity (AQG) I. Conceptual Setup, 2007 Class. Quant. Grav. 24, 2465 [gr-qc/0607099], Algebraic Quantum Gravity (AQG) II. Semiclassical Analysis, 2007 Class. Quant. Grav. 24, 2499 [gr-qc/0607100], Algebraic Quantum Gravity (AQG) III. Semiclassical Perturbation Theory, 2007 Class. Quant. Grav. 24, 2565 [gr-qc/0607101]

[15] Ashtekar, A., Lewandowski, J.: Differential Geometry on the Space of Connections via Graphs and Projective Limits 1995 J.Geom.Phys. 17 191-230 [arXiv:hepth/9412073]

[16] Fleischhack, C.: Hyphs and the Ashtekar-Lewandowski measure 2003 Jour. Geom. Phys. 45231 [MIS-preprint 3/2000]

[17] Bratteli, O., Robinson, J.: Operator algebras and Quantum Statistical Mechanics I, 2nd edition 1987 Springer Verlag Heidelberg

[18] Shalmann, H.: Exploring the diffeomorphism invariant Hilbert space of a scalar field 2007 Classical and Quantum Gravity, 24, 4601-4615 [arXiv:gr-qc/0609032] 
[19] Freidel, L., Livine, E. Spin Networks for Non-Compact Groups 2003 J.Math.Phys. 441322 [arXiv:hep-th/0205268]

[20] Rovelli, C.: Partial observables 2002 Phys.Rev. D 65124013 [arXiv:grqc/0110035], Dittrich, B.: Partial and Complete Observables for Hamiltonian Constrained Systems 2006 Class.Quant.Grav. 23 6155-6184 [arXiv:grqc/0507106]

[21] Thiemann, T. The Phoenix Project: Master Constraint Programme for Loop Quantum Gravity 2006 Class. Quant. Grav. 23, 2211 [arXiv:gr-qc/0305080], Thiemann, T. Quantum Spin Dynamics VIII. The Master Constraint 2006 Class. Quant. Grav. 23, 2249 [arXiv:gr-qc/0510011]

[22] Baez, J.: Spin Foam Models 1998 Class.Quant.Grav. 15 1827-1858 [arXiv:grqc/9709052]

[23] Pfeiffer, H,: Higher gauge theory and a non-Abelian generalization of 2-form electrodynamics 2003 Annals Phys. 308 447-477 [arXiv:hep-th/0304074], Girelli, F., Pfeiffer, H.: Higher gauge theory - differential versus integral formulation, J.Math.Phys. 200445 3949-3971 [arXiv:hep-th/0309173]

[24] Mackaay, M.: Spherical 2-categories and 4-manifold invariants Adv. Math. 1999 143, 288-348 [arXiv:math/9805030]

[25] Adámek, Jiří, Herrlich, Horst, Strecker, George E. Abstract and concrete categories (1990) John Wiley and Sons

[26] Magnus, W., Karrass, A., Solitar, D.: Combinatorial Group theory: Presentations of Groups in Terms of Generators and Relations 1976 Dover Publications, Inc. (New York)

[27] Fleischhack, C.: Construction of Generalized Connections [arXiv:math$\mathrm{ph} / 0601005]$ 